\title{
USO DE MÉTODOS BAYESIANOS PRECISOS EM MODELOS DE REGRESSÃO COM DADOS DE SOBREVIVÊNCIA
}

\author{
Vera Lucia Damasceno
}

Orientador

Prof. Dr. Jorge Alberto Achcar

Dissertação apresentada ao Instituto de Ciências Matemáticas de São Carlos-USP, como parte dos requisitos para obtenção do titulo de mestre em "Ciências de Computação e Matemática Computacional".

São Carlos 


\section{"A educação é a caminhada do homem da ignorância excessivamente confiante para a incerteza refletida"}

\section{Don Clark}

$$
\begin{array}{r}
\text { A minha familia } \\
\text { em especial } \\
\text { a minha mãe } \\
\text { Damasceno } \\
\text { deorgina a meu marido } \\
\text { doão Nivaldo Jomazella } \\
\text { pelo apoio e carinho. }
\end{array}
$$




\section{Agradecimentos}

A conclusão dessa dissertação não seria possível sem o auxílio de algumas pessoas e instituições que contribuíram de alguma forma para este projeto, às quais expresso os mais sinceros agradecimentos.

A Deus por mais esta realização em minha vida.

Ao Prof. Dr. Jorge Alberto Achcar pela sua orientação dedicada e segura, participando ativamente do estudo, incentivando-me em todo o decorrer da pesquisa.

Ao Prof. Dr. Josemar Rodrigues e ao Prof. Dr. Carlos Diniz pelas valiosas sugestões e comentários dados quando da realização do meu exame de qualificação.

Aos colegas e funcionários do Departamento de Matemática da Universidade Federal do Maranhão (UFMA) pelo apoio e incentivo e a UFMA pela concessão do afastamento integral para a realização dos meus estudos.

A Profa. Dra. Maria Creusa Bretas Salles pelo apoio e incentivo ao meu ingresșo na pós-graduação.

A FAPESP e a CAPES pelo apoio financeiro.

Ao amigo José Carlos Fogo pelo companheirismo e ajuda computacional nos meus estudos.

A todos aqueles que de alguma maneira contribuiram para a realização desse trabalho. Aos professores, funcionários e colegas de pós-graduação, em especial aos amigos, Adriana, Carlos Aparecido, Cecília, Elisa, Eric, Esdras, Flávio, Gaby, Iêda, Janete, Josmar, Lilian, Mazé, Milton, Mercadante, Pérsida, Sadao, Silvana, Tereza Cristina, pela amizade e apoio durante este periodo, dentro e fora do ICMSC. 


\section{Resumo}

Nesta dissertação, considerando um modelo de regressão exponencial para dados de tempo de vida com uma covariável $x$ proposta por Feigl e Zelen (1965) e um modelo de regressão locação-escala com distribuição valor extremo, desenvolvemos uma análise Bayesiana. Consideramos, nestas análises, dados sob um esquema de amostragem completa e densidades a priori não-informativas de Jeffreys para os parâmetros envolvidos. Alguns aspectos de reparametrizações são usados onde exploramos uma forma modificada para a parametrização proposta por Guerrero e Johnson (1982). Também exploramos uma medida de não normalidade para a função de verossimilhança e densidade a posteriori de interesse, originalmente proposta por Sprott (1973). Os métodos de aproximações de integrais e os métodos numéricos são usados para aproximar densidades a posteriori marginal e momentos a posteriori de interesse quando não conseguimos encontrar soluções analíticas explícitas. Também usamos o "t-plot" proposto por Hills e Smith (1993) para verificar a adequabilidade da reparametrização proposta. 


\section{Abstrat}

In this work, considering an exponential regresion model for lifetime data with a covariate $x$ introduced by Feigl and Zelen (1965) and a location-scale regression model with an extreme value distribution, we present a Bayesian analysis. We consider in these analyses, complete lifetime data and Jeffreys noninformative prior densities for the parameters. Some aspects of reparametrization are considered, where we explore a modified form for a parametrization introduced by Guerrero e Johnson (1982). We also explore a nonnormality measure for likelihood functions and posterior densities of interest introduced by Sprott (1973). Approximation methods and numerical methods for integrals are used to approximate marginal posterior densities and posterior moments of interest, when we cannot obtain explicit analytical solutions. We also use the "t-plot" introduced by Hills and Smith (1993) to verify the adequability of the proposed reparametrization. 


\section{ÍNDICE}

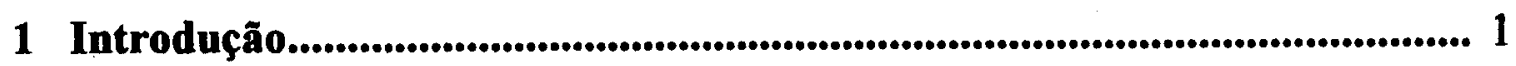

1.1 Modelo de Riscos Proporcionais .......................................................... 4

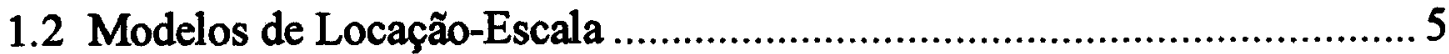

1.3 Modelo de Regressão Exponencial ....................................................

1.4 Modelo de Regressão de Weibull e de Valor Extremo ........................ 12

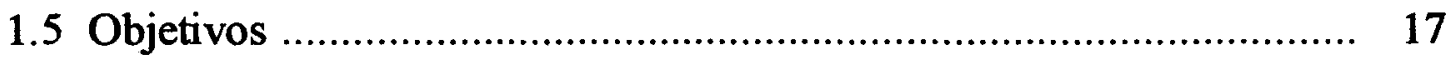

2 Alguns Métodos de Resolução de Integrais em Inferência Bayesiana ...... 19

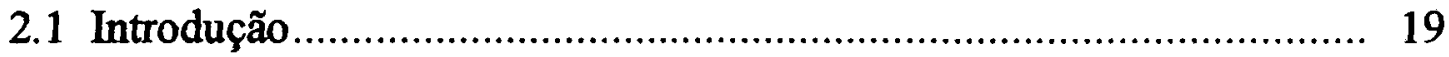

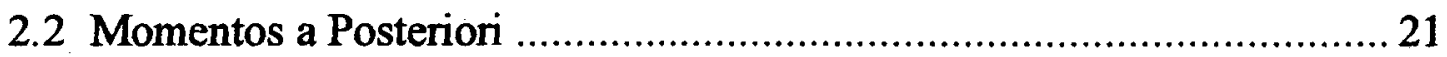

2.2.1 Aproximação de Tierney e Kadane.......................................... 21

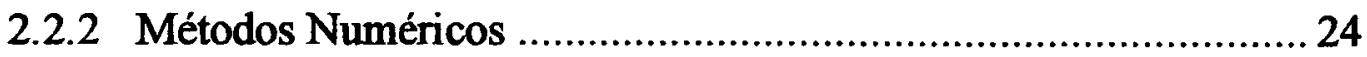

2.2.3 Procedimento de Monte Carlo .................................................... 27

2.3 Densidades a posteriori Marginais................................................. 29

2.3.1 Aproximação de Tierney, Kass e Kadane (TKK) ....................... 30

2.3.2 Aproximação de Leonard, Hsu e Tsui (LHT) ............................... 32

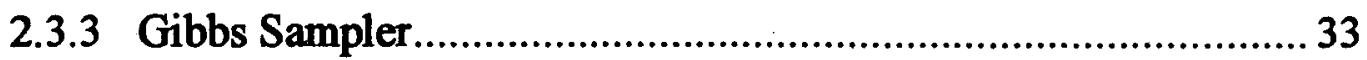

3 Alguns Aspectos de Reparametrização …………...................................................... 35

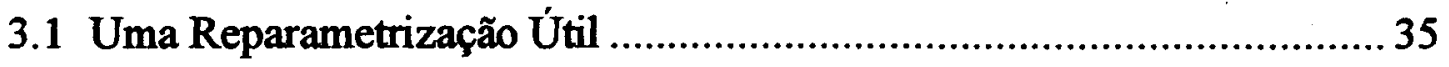

3.2 Uma Medida Diagnóstica de Não- Normalidade ……………................38 
3.2.1 Derivada Terceira Padronizada (STD)

3.3 Reparametrização de Guerrero e Johnson para a Função de

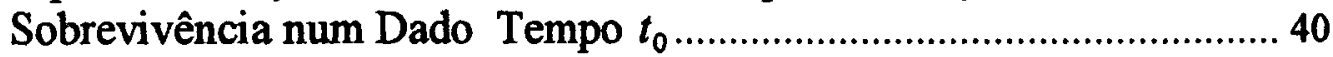

3.4 Diagnóstico Gráfico para Verificar uma Parametrização ......................... 42

4 Modelo de Regressão de Feigl e Zelen ..................................................4

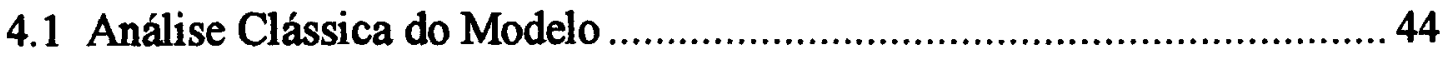

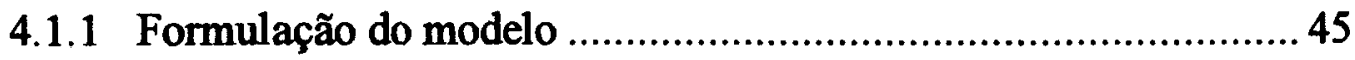

4.1.2 Aproximação Normal para os Estimadores de Máxima Verossimilhança ................................................................. 46

4.1.3 Reparametrização de Guerrero e Johnson para a função de Sobrevivência.

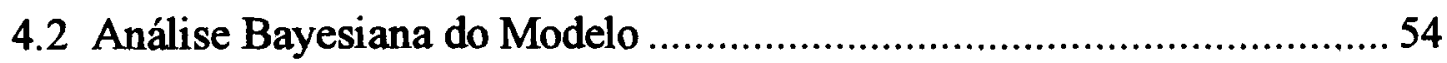

4.2.1 Uma Densidade a Priori Não-Informativa para $\theta_{1}$ e $\theta_{2} \ldots \ldots \ldots \ldots . . .55$

4.2.2 Densidade à Posteriori Conjunta para $\theta_{1}$ e $\theta_{2} \ldots \ldots \ldots \ldots \ldots \ldots \ldots \ldots . . .57$

4.2.3 Densidade a Posteriori Marginal para a Função de

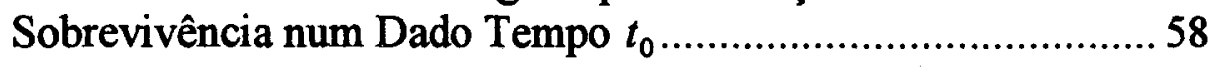

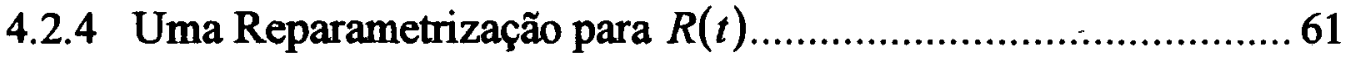

\section{Modelo de Regressão Locação-Escala com Distribuição de}

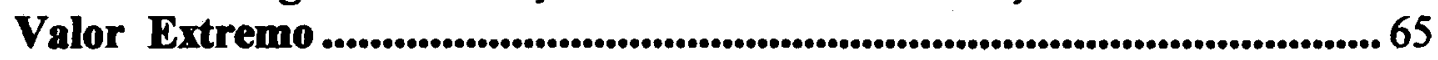

5.1 Aproximação Normal para os Estimadores de Máxima

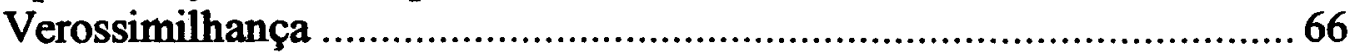

5.2 Estimação da Função de Sobrevivência ..................................................68

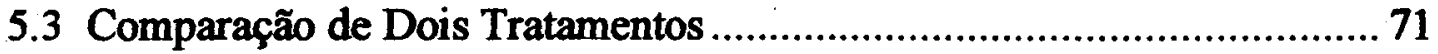

5.3.1 Reparametrização de Guerrero e Johnson para a Função de Sobrevivência 
5.3.2 Análise Bayesiana Assumindo $\beta_{0}, \beta_{1}$ e $\sigma$ Desconhecidos.

5.3.2.1 Densidades a Priori e a Posteriori ................................... 77

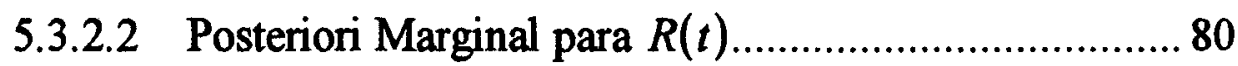

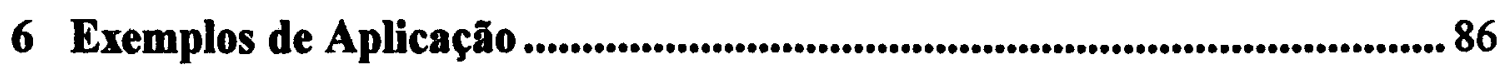

6.1 Um Exemplo com o Modelo de Regressão de Feigl e Zelen. ……........... 86

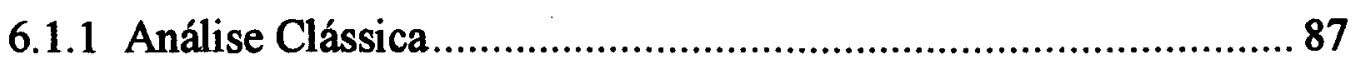

6.1.1.1 Verificação da Normalidade da Função de

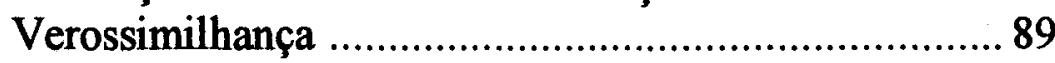

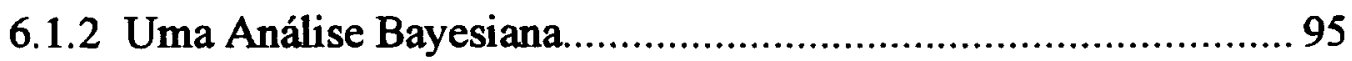

6.2 Um Exemplo com a Distribuição de Valor Extremo .......................... 103

6.2.1 Comparação de Dois Tratamentos ………………................. 103

6.2.1.1 Análise Clássica ................................................... 104

6.2.1.1.1 Verificação da Normalidade da Função de Verossimilhança .......................................... 107

6.2.1.2 Uma Análise Bayesiana....................................... 113

7 Considerações Finais e Perspectivas Futuras ...................................................... 118

Apêndice A.1 Derivadas do Logaritmo da Função de Verossimilhança "Profile" para $\phi_{G J}(\lambda)$ - Caso Exponencial (Modelo de Feigl e Zelen) ............................................................ 120

Apêndice A.2 Obtenção de Intervalo HPD ............................................ 122

Apêndice A.3 Listagem de Programas de Computador ........................... 123

Referências ......................................................................................................................... 133 


\section{CAPÍTULO 1}

\section{INTRODUÇÃO}

A análise estatística para dados de sobrevivência tem sido utilizada em muitas áreas de aplicação, especialmente em engenharia e medicina onde, os pesquisadores usualmente estâo interessados em analisar dados relacionados aos tempos de vida de unidades. Essas unidades podem ser, por exemplo, peças de um determinado equipamento ou pacientes submetidos a um certo tratamento. Neste último caso, o pesquisador pode estar interessado no tempo de vida até à morte de um paciente ou na sua recuperação.

Na prática muitas situações envolvem populações heterogêneas e é importante considerar a relação entre os tempos de sobrevivência com outros fatores. Um modo de fazer isso é através de um modelo de regressão, onde a dependência do tempo de sobrevivência com a covariável é explicitamente identificada. Nessas situações a literatura estatística apresenta muitas técnicas apropriadas para a análise de dados desta natureza, tais como: técnicas de regressão não paramétricas (ver por exemplo, Miller, 1976; Buckley e James, 1979; Lawless,1982; Cox, 1972a; Koul, Susarla e Van Ryzin, 1981; Schmee e Hahn,1979) e várias técnicas de regressão paramétricas (ver por exemplo, Feigl e Zelen, 1965; ou Zippin e Armitage, 1966).

Neste trabalho damos ênfase ao caso paramétrico e vamos assumir que existe um modelo paramétrico que descreve adequadamente a distribuição dos tempos de vida. Algumas dessas técnicas paramétricas assumem usualmente o modelo exponencial para 0 
tempo de sobrevivência, como por exemplo as técnicas de Feigl e Zelen, 1965; de Glasser, 1967 e de Zippin e Armitage, 1966.

A formulação dos modelos paramétricos deve levar em conta que a variável de interesse é representada por uma variável aleatória não negativa. Esta restrição tem influência sobre a escolha da forma pela qual se relacionam as variáveis independentes com as dependentes. Dois tipos de abordagem têm sido utilizadas para tratar o problema. A primeira é baseada na utilização de modelos de riscos proporcionais. A segunda consiste na utilização de transformações, em particular, a logarítimica dos tempos de vida, que fornece modelos do tipo locação-escala.

Neste trabalho será desenvolvido dois casos especiais os quais pertencem às duas classes de modelos de regressão: o modelo de regressão proposto por Feigl e Zelen (1965), que assume uma distribuição exponencial para o tempo de sobrevivência $T$, e o modelo de valor extremo que assume uma distribuição de Weibull para o tempo de sobrevivência $T$.

Em engenharia, em geral o objetivo principal é a análise da confiabilidade de um componente (produto). Em particular, uma quantidade básica de interesse é a confiabilidade no tempo de sobrevivência do componente sob condições normais de funcionamento em que usualmente é submetido, isto é, nas condições ambientes em que o componente em questão é submetido à operação. Em análise de dados médicos geralmente o pesquisador tem interesse em obter inferência sobre a função de sobrevivência de um paciente em um tempo especificado $t_{0}$. Nessas situações é comum a presença de um ou mais fatores o qual pode afetar o tempo de vida de unidade, assim é requerido o uso de um modelo de regressão. 
Um problema de grande interesse médico é a comparação de dois tratamentos. Em geral o pesquisador quer verificar se um tratamento novo é superior a um tratamento tradicional. Com dados de sobrevivência tendo uma distribuição de Weibull, apresentamos neste trabalho uma análise de regressão com dados transformados para uma distribuição de valor extremo, na comparação de dois tratamentos.

Também são adotados aspectos de reparametrização com o intuito de obtermos boa "normalidade" da função de verossimilhança ou densidade a posteriori de interesse, que implicam, em geral em uma boa precisão nos resultados assintóticos.

Em situações práticas de testes de vida, os dados de sobrevivência, em geral, são incompletos ou censurados. Isto pode ser devido ao próprio planejamento do experimento considerado para a coleta dos dados.

Um plano de amostragem muito usado na área de engenharia é dado pelo esquema de censuras de tipo II, onde sob as condições ambientais, o teste é aplicado aos componentes e termina quando um número pré - determinado de falhas é obtido.

No esquema de amostragem completa, todos os componentes da amostra são observados até que todos falhem. Para este plano amostral, as inferências são análogas ao esquema de censuras do tipo II.

Um outro esquema de censuras é dado pelo esquema de censuras de tipo I. Neste esquema, são observados os tempos de sobrevivência de $n$ unidades durante um tempo fixo $t_{0}$. Este plano amostral tem grande aplicação na área médica (ver por exemplo Lawless,1982). 
Nas seções seguintes apresentamos uma breve revisão de resultados sob o enfoque clássico desses modelos de regressão que têm sido constantemente explorados na literatura (ver por exemplo, Lawless,1982).

\subsection{Modelo de Riscos Proporcionais}

Uma família de riscos proporcionais é uma classe de modelos com a propriedade que diferentes indivíduos têm funções de risco proporcionais entre si, isto é, para dois indivíduos com vetores de covariáveis $\mathbf{x}_{1}$ e $\mathbf{x}_{2}$, a razão $h\left(t \mid \mathbf{x}_{1}\right) / h\left(t \mid \mathbf{x}_{2}\right)$ não varia com $t$. Isto implica que os modelos de riscos proporcionais são dados por:

$$
h(t \mid \mathbf{x})=h_{0}(t) g(\mathbf{x})
$$

onde $h(t \mid \mathbf{x})$ é a função de risco dado $\mathbf{x}, \mathbf{x}$ é um vetor de covariáveis $1 \times p$ e $h_{0}(t)$ é a função de risco padrão quando $g(\mathbf{x})=1$. As funções $h_{0}$ e $g$ podem envolver parâmetros desconhecidos.

Um caso particular da família de modelos de riscos proporcionais é proposto por Cox (1972), com

$$
h(t \mid \mathbf{x})=h_{0}(t) e^{\mathbf{x} \beta}
$$


onde $\beta=\left(\beta_{1}, \beta_{2}, \ldots, \beta_{p}\right)$ é o vetor dos coeficientes de regressão. Os coeficientes de regressão $\beta$ e a função de risco $h_{0}(t)$ são desconhecidos.

Com o modelo (1.2), a função de sobrevivência $R(t \mid \mathbf{x})=1-F(t \mid \mathbf{x})$ é dada por:

$$
R(t \mid \mathbf{x})=\exp \left\{-\int_{0}^{t} h(u \mid \mathbf{x}) d u\right\}=\exp \left\{-e^{\mathbf{x} \beta} \int_{0}^{t} h_{0}(u) d u\right\}=\left\{R_{0}(t)\right\}^{e^{\mathrm{x} \beta}}
$$

onde $R_{0}(t)=\exp \left\{-\int_{0}^{t} h_{0}(u) d u\right\}$ é a função de sobrevivência padrão com $g(\mathbf{x})=1$.

Observamos que (1.1) estabelece uma relação de ordem entre as funções de sobrevivências, isto é, para dois indivíduos com covariáveis $\mathbf{x}_{1}$ e $\mathbf{x}_{2}$, temos:

$$
R\left(t \mid \mathbf{x}_{1}\right) \geq R\left(t \mid \mathbf{x}_{2}\right), \text { para todo } t
$$

onde, $g\left(\mathbf{x}_{2}\right) \geq g\left(\mathbf{x}_{1}\right)$.

\subsection{Modelos de Locação-Escala}

Supor que os tempos de vida $T$ são transformados na forma $Y=\log T$. A classe dos modelos de locação-escala corresponde àquela para a qual, dado $\mathbf{x}$, a variável $Y$ tem uma distribuição com um parâmetro de locação $\mu(\mathbf{x})$ que introduz as covariadas no modelo e um parâmetro de escala $\sigma \geq 0$ constante. Estas quantidades relacionam-se através da expressão: 


$$
Y=\mu(\mathbf{x})+\sigma e,
$$

onde $e$ tem uma distribuição que é independente de $\mathbf{x}$.

Em geral $\mu(x)=x^{\prime} \beta$. Note que nos modelos de locação-escala as variáveis independentes atuam linearmente sobre $\mathrm{Y}$ e portanto multiplicativamente sobre $T$, ao passo que nos modelos com riscos proporcionais $\mathbf{x}$ atua multiplicativamente sobre a função de risco de $T$ (ver 1.1).

A função de sobrevivência para $Y$, dado $\mathbf{x}$, é da forma:

$$
G\{[y-\mu(\mathbf{x})] / \sigma\}
$$

onde $G(e)$ è a função de sobrevivência de $e$.

A função de sobrevivência para $T=\exp Y$ é dada por:

$$
R(t \mid \mathbf{x})=G\left(\frac{\log T-\mu(\mathbf{x})}{\sigma}\right)=R_{1}\left[\left(\frac{t}{\alpha(\mathbf{x})}\right)^{\delta}\right]
$$

onde $\alpha(\mathrm{x})=\exp [\mu(\mathrm{x})], \delta=1 / \sigma$, e $R_{1}(w)=G(\log w)$.

O modelo exponencial e o de Weibull são casos particulares destas duas famílias de modelos. 


\subsection{Modelo de Regressão Exponencial}

Supor que as unidades tenham uma função de risco constante que pode depender de uma ou mais covariáveis. Neste caso o modelo de regressão exponencial é apropriado, e a f.d.p. para o tempo de sobrevivência $T$, dada a covariável $\mathbf{x}$, é dada por:

$$
f(t \mid \mathbf{x})=\theta_{\mathbf{x}}^{-1} \exp \left(\frac{-t}{\theta_{x}}\right) \quad t>0
$$

onde $\mathbf{x}$ pode ser um vetor de covariáveis independentes e $\theta_{\mathbf{x}}=E[T \mid \mathbf{x}]$.

Várias formas funcionais para $\theta_{\mathbf{x}}$ são possíveis, em (1.7). Um caso especial é dado por,

$$
\theta_{\mathrm{x}}=e^{\mathrm{x} \beta}
$$

onde $\mathrm{x}=\left(x_{1}, \ldots, x_{p}\right)$ e $\beta^{\prime}=\left(\beta_{1}, \ldots, \beta_{p}\right)$ é um vetor de parâmetros de um modelo de regressão. Esta forma de modelo foi introduzida por Glasser (1967), Cox e Snell (1968), Prentice (1973), Lawless (1976) e outros.

Uma vantagem da forma (1.8) é que $\theta_{\mathbf{x}}>0$ é automaticamente satisfeita para todo $\mathrm{x}$ e $\beta$.

Outras formas também usadas na literatura são dadas por:

i) $\theta_{x}=x \beta$

(ver por exemplo Feigl e Zelen,1965; Zippin e Armitage; 1966) 
ii) $\theta_{\mathbf{x}}=(\mathbf{x} \beta)^{-1}$

(ver por exemplo, Greenberg et al., 1974).

O modelo (1.7) é um modelo de Risco Proporcional mas também pode ser visto como um modelo de locação-escala na transformação $Y=\log T$. De (1.7) e (1.8) a f.d.p. de $Y$, dado $\mathbf{x}$ é dada por:

$$
f(y \mid \mathbf{x})=\exp \{(y-\mathbf{x} \beta)-\exp (y-\mathbf{x} \beta)\},-\infty<y<\infty
$$

Assim, podemos escrever de (1.9) o modelo de locação-escala $y=\mathbf{x} \beta+z$, onde $z$ tem uma distribuição de valor extremo padronizada com a f.d.p. $f(z)=\exp \left(z-e^{z}\right)$, $-\infty<z<\infty$.

A função de verossimilhança para $\beta$, sob o modelo (1.9) e dados censurados para $n$ individuos, é dada por:

$$
L(\beta)=\prod_{i \in D} f(y \mid \mathbf{x}) \prod_{i \in C} R(y \mid \mathbf{x})
$$

onde $f(y \mid \mathbf{x})$ é dada em (1.9) e

$$
R(y \mid \mathbf{x})=\exp [-\exp (y-\mathbf{x} \beta)]
$$

As notações $i \in D$ e $i \in C$ são usadas para denotar individuos $i$ para os quais $t_{i}$ é um tempo de vida ou um tempo de censura, respectivamente. 
Substituindo (1.9) e (1.11) na equação (1.10), a função de verossimilhança para $\beta$ é dada por:

$$
L(\beta)=\prod_{i \in D} \exp \left[\left(y_{i}-\mathbf{x}_{i} \beta\right)-\exp \left(y_{i}-\mathbf{x}_{i} \beta\right)\right] \prod_{i \in C} \exp \left[-\exp \left(y_{i}-\mathbf{x}_{i} \beta\right)\right]
$$

Os estimadores de máxima verossimilhança dos componentes do vetor $\beta$ são dados por:

$$
\frac{\partial \ell(\beta)}{\partial \beta_{r}}=0 \quad r=1, \ldots, p,
$$

onde $\ell(\beta)=\ln L(\beta)$ é dado por:

$$
\begin{aligned}
\ell(\beta)= & \ln \left\{\prod_{i \in D} \exp \left[\left(y_{i}-x_{i} \beta\right)-\exp \left(y_{i}-\mathbf{x}_{i} \beta\right)\right] \prod_{i \in C} \exp \left[-\exp \left(y_{i}-\mathbf{x}_{i} \beta\right)\right]\right\} \\
& =\sum_{i \in D}\left(y_{i}-\mathbf{x}_{i} \beta\right)-\sum_{i=1}^{n} \exp \left(y_{i}-\mathbf{x}_{i} \beta\right) .
\end{aligned}
$$

Portanto, os estimadores de máxima verossimilhança de $\beta_{r}, r=1, \ldots, p$ são dados pela solução das equações:

$$
\frac{\partial \ell(\beta)}{\partial \beta_{r}}=-\sum_{i \in D} x_{i r}+\sum_{i=1}^{n} x_{i r} \exp \left(y_{i}-x_{i} \beta\right)=0, r=1, \ldots, p
$$


Para solucionar numericamente as equações de verossimilhança (1.15) devemos recorrer a métodos iterativos.

$O$ estimador de máxima verossimilhança $\hat{\beta}$ para uma amostra aleatória de tamanho $n$ com dados com censuras de tipo II tem uma distribuição normal assintótica dada por:

$$
\hat{\boldsymbol{\beta}} \sim N\left(\boldsymbol{\beta}, I^{-1}\right)
$$

onde $I$ é matriz de informação de Fisher, dada por:

$$
I=E\left(-\frac{\partial^{2} \log L}{\partial \beta_{r} \partial \beta_{s}}\right)=\sum_{i=1}^{n} x_{i r} x_{i s}\left[1-\exp \left(-L_{i} e^{-x_{i} \beta}\right)\right] \quad r, s=1, \ldots, p
$$

onde $L_{i}$ são os tempos de censuras.

Note que $I^{-1}$, o inverso da matriz de informação de Fisher, é a matriz de variância e covariância assintótica de $\hat{\beta}$ ( ver por exemplo, Bickel e Doksum, 1977).

Testes de significância e estimativas por intervalos para $\beta$ podem ser baseados no método da razão de verossimilhança ou numa aproximação normal para $\hat{\beta}$. Na prática é mais conveniente usar $\hat{\boldsymbol{\beta}} \sim N\left(\boldsymbol{\beta}, I_{0}^{-1}\right)$, onde $I_{0}=\left(-\partial^{2} \log L / \partial \beta_{r} \partial \beta_{s}\right)_{\hat{\beta}}$ é a matriz de informação observada.

Considere problemas de testes de hipóteses na forma $H_{0}: \beta_{1}=\beta_{1}^{0}$, onde $\beta$ é particionado por $\left(\beta_{1}, \beta_{2}\right)^{\prime}, \beta_{1}$ é $(k \times 1)(k<p)$ e $\beta_{1}^{0}$ é um vetor especificado. Para testar $H_{0}$ contra $H_{1}: \beta_{1} \neq \beta_{1}^{0}$ poderíamos usar a estatística da razão de verossimilhanças, 


$$
\Lambda=-2 \log \left(\frac{L\left(\beta_{1}^{0}, \tilde{\beta}_{2}\right)}{L\left(\hat{\beta}_{1}, \hat{\beta}_{2}\right)}\right),
$$

onde $\tilde{\beta}_{2}$ é o EMV de $\beta_{2}$ sob a hipótese $H_{0}$ e $\hat{\beta}=\left(\hat{\beta}_{1}, \hat{\beta}_{2}\right)$ é o EMV de $\beta$ sem restrição. $O$ estimador $\tilde{\beta}_{2}$ é encontrado resolvendo o sistema de equações $\partial \log L / \partial \beta_{r}=0$ $(r=k+1, \ldots, p)$ com $\beta_{1}$ igual a $\beta_{1}^{0}$. Valores grandes de $\Lambda$ evidenciam contra $H_{0}$. Para uma amostra de tamanho grande, o nível de significância pode ser calculado por uma aproximação assintótica $\Lambda \stackrel{a}{\sim} \chi_{k}^{2}$.

Uma estatística alternativa para testar $H_{0}$, baseada na aproximação normal assintótica $\hat{\boldsymbol{\beta}} \stackrel{a}{\sim} N\left(\beta, I_{0}^{-1}\right)$ é dada por:

$$
\Lambda_{1}=\left(\hat{\beta}_{1}-\beta_{1}^{0}\right)^{\prime} C_{11}^{-1}\left(\hat{\beta}_{1}-\beta_{1}^{0}\right)
$$

onde $C_{11}$ é uma matriz $(k \times k)$ de covariância assintótica para $\hat{\beta}_{1}$ sob $H_{0}$ e $C=I_{0}^{-1}$ é particionada por:

$$
C=\left(\begin{array}{ll}
C_{11} & C_{12} \\
C_{12} & C_{22}
\end{array}\right)
$$

Para amostras grandes sob $H_{0}, \Lambda_{1}$ é também aproximadamente $\chi_{k}^{2}$. A aproximação $\chi^{2}$ para $\Lambda$ é em geral melhor para amostras médias ou pequenas do que a aproximação $\dot{\chi}^{2}$ para $\Lambda_{1}$. 
Em geral, a precisão desses resultados assintóticos é discutível, especialmente para amostras pequenas ou moderadas. Uma alternativa para melhorar estes resultados, é considerar reparametrizações que melhorem a normalidade da função de verossimilhança (ver por exemplo Anscombe, 1964 ou Sprott, 1973).

Análises Bayesianas para o modelo (1.9), também tem sido consideradas por vários autores, (ver por exemplo Achcar e Bolfarine, 1986; Martz e Waller, 1982; ou Barlow e Proschan, 1979 ).

\subsection{Modelo de Regressão de Weibull e de Valor Extremo}

A distribuição de Weibull é mais flexível do que o modelo exponencial pois podemos incluir várias formas de risco. A f.d.p. de Weibull é dada por:

$$
f(t)=\frac{\delta}{\alpha}\left(\frac{t}{\alpha}\right)^{\delta-1} \exp \left[-\left(\frac{t}{\alpha}\right)^{\delta}\right] \quad t \geq 0
$$

onde $\delta>0$ e $\alpha>0$ são parâmetros de forma e escala, respectivamente (ver por exemplo Lawless, 1982).

A f.d.p. do tempo de vida, dado o vetor $\mathbf{X}$ de variáveis regressoras é dada por:

$$
f(t)=\frac{\delta}{\alpha(\mathbf{x})}\left(\frac{t}{\alpha(\mathbf{x})}\right)^{\delta-1} \exp \left[-\left(\frac{t}{\alpha(\mathbf{x})}\right)^{\delta}\right], t \geq 0
$$


Observe que estamos assumindo que somente o parâmetro de escala $\alpha$ depende de $\mathbf{x}$. Em particular, o fato de $\delta$ não depender de $\mathbf{x}$ implica risco proporcional para o tempo de vida e variância constante para o logaritmo do tempo de vida dos indivíduos (ver por exemplo, Peto e Lee, 1973 ).

Muitas vezes, em lugar da distribuição de Weibull é mais conveniente trabalharmos com a forma da distribuição de valor extremo, onde a f.d.p. de $Y=\log T$ é dada por:

$$
f(y ; \mu, b)=\frac{1}{b} \exp \left\{\frac{(y-\mu)}{b}\right\} \exp \left\{-\exp \left(\frac{y-\mu}{b}\right)\right\},
$$

onde $-\infty<y<\infty,-\infty<\mu<\infty$ e $b>0$. Se $T$ tem f.d.p. (1.20), então $Y=\log T$ tem f.d.p. (1.22) $\operatorname{com} \mu=\log \alpha$ e $b=\delta^{-1}$.

$$
\begin{aligned}
& \text { A f.d.p. de } Y=\log T \text {, dado o vetor } \mathbf{x}, \text { é da forma: } \\
& f(y \mid \mathbf{x})=\frac{1}{\sigma} \exp \left[\frac{y-\mu(\mathrm{x})}{\sigma}-\exp \left(\frac{y-\mu(\mathbf{x})}{\sigma}\right)\right]-\infty<y<\infty,
\end{aligned}
$$

onde $\mu(x)=\log \alpha(x)$ e $\sigma=\frac{1}{\delta}$. O modelo de regressão mais frequentemente usado é dado na forma linear,

$$
\mu(\mathbf{x})=\mathbf{x} \beta,
$$


onde $\mathrm{x}=\left(x_{1}, \ldots, x_{p}\right)$, é o vetor de dimensão $p$ de variáveis regressoras e $\beta=\left(\beta_{1}, \ldots, \beta_{p}\right)^{\prime}$ é o vetor dos coeficientes de regressão.

Com $\mu(x)=x \beta$, podemos escrever o modelo na forma,

$$
y=\mathbf{x} \beta+\sigma z
$$

onde $z$ tem uma distribuição de valor extremo padronizada com f.d.p. dada por $f(z)=\exp \left(z-e^{z}\right),-\infty<z<\infty$. A constância de $\delta$ em (1.20) corresponde a constância de $\sigma \mathrm{em}(1.23)$.

Considerando o modelo dado em (1.23) com $n$ unidades em testes, a função de verossimilhança para $\beta$ e $\sigma$ é dada por:

$$
L(\beta, \sigma)=\prod_{i \in D} \frac{1}{\sigma} \exp \left[\left(\frac{y_{i}-\mathbf{x}_{i} \beta}{\sigma}\right)-\exp \left(\frac{y_{i}-\mathbf{x}_{i} \beta}{\sigma}\right)\right] \prod_{i \in C} \exp \left[-\exp \left(\frac{y_{i}-\mathbf{x}_{i} \beta}{\sigma}\right)\right],
$$

onde a notação $C$ e $D$ foi definida na seção 1.3 .

O logaritmo da função de verossimilhança para $\beta$ e $\sigma$ é dado por:

$$
\ell(\beta, \sigma)=-r \log \sigma+\sum_{i \in D}\left(\frac{y_{i}-\mathbf{x}_{i} \beta}{\sigma}\right)-\sum_{i}^{n} \exp \left(\frac{y_{i}-\mathbf{x}_{i} \beta}{\sigma}\right)
$$

onde $r$ é o número observado de tempos de vida. 
Com $z_{i}=\left(y_{i}-x_{i} \beta\right) / \sigma$, os estimadores de máxima verosimilhança de $\beta$ e $\sigma$ são dados pela solução do sistema de equações:

$$
\left\{\begin{array}{l}
\frac{\partial \log L}{\partial \beta_{l}}=-\frac{1}{\sigma} \sum_{i \in D} x_{i l}+\frac{1}{\sigma} \sum_{i=1}^{n} x_{i l} e^{z_{i}}=0 \quad l=1, \ldots, p \\
\frac{\partial \log L}{\partial \sigma}=-\frac{r}{\sigma}-\frac{1}{\sigma} \sum_{i \in D} z_{i}+\frac{1}{\sigma} \sum_{i=1}^{n} z_{i} e^{z_{i}}=0
\end{array}\right.
$$

Para solucionarmos numericamente as equações (1.26) podemos recorrer a métodos iterativos, como por exemplo o método iterativo de Newton-Raphson.

A matriz de informação observada $I_{0}((p+1) \times(p+1))$ numa forma particionada é dada por:

$$
I_{0}=-\left(\begin{array}{ll}
\frac{\partial^{2} \log L}{\partial \beta_{l} \partial \beta_{s}} & \frac{\partial^{2} \log L}{\partial \beta_{l} \partial \sigma} \\
\frac{\partial^{2} \log L}{\partial \beta_{s} \partial \sigma} & \frac{\partial^{2} \log L}{\partial \sigma^{2}}
\end{array}\right)_{(\hat{\hat{\beta}}, \hat{\mathrm{\sigma}})}
$$

onde as derivadas segundas são dadas por: 


$$
\left\{\begin{array}{l}
\frac{\partial^{2} \log L}{\partial \beta_{l} \partial \beta_{s}}=-\frac{1}{\sigma^{2}} \sum_{i=1}^{n} x_{i l} x_{i s} e^{z_{i}} \quad l, s=1, \ldots, p \\
\frac{\partial^{2} \log L}{\partial \sigma^{2}}=\frac{r}{\sigma^{2}}+\frac{2}{\sigma^{2}} \sum_{i \in D} z_{i}-\frac{2}{\sigma^{2}} \sum_{i=1}^{n} z_{i} e^{z_{i}}-\frac{1}{\sigma^{2}} \sum_{i=1}^{n} z_{i}^{2} e^{z_{i}} \\
\frac{\partial^{2} \log L}{\partial \beta_{l} \partial \sigma}=\frac{1}{\sigma^{2}} \sum_{i \in D} x_{i l}-\frac{1}{\sigma^{2}} \sum_{i=1}^{n} x_{i l} e^{z_{i}}-\frac{1}{\sigma^{2}} \sum_{i=1}^{n} x_{i l} z_{i} e^{z_{i}} \quad l=1, \ldots, p
\end{array}\right.
$$

Testes de hipóteses e estimativas por intervalos para os parâmetros podem ser obtidos usando o método da razão de verosimilhança ou uma aproximação normal para os estimadores de máxima verosimilhança em amostras grandes (ver Lawless, 1982).

Para uma amostra aleatória de tamanho $n$ grande, se $\hat{\beta}$ e $\hat{\sigma}$ são estimadores de máxima verossimilhança de $\beta$ e $\sigma$, temos,

$$
(\hat{\beta}, \hat{\sigma})^{a} \sim N\left((\beta, \sigma), I_{0}^{-1}\right)
$$

Novamente, enfatizamos que o uso desta distribuição assintótica deve ser feita com cuidado, especialmente para amostras pequenas.

Consideramos problemas onde hipóteses podem ser feitas sobre $\beta$ na seguinte forma: $H_{0}: \beta_{1}=\beta_{1}^{0}$ onde $\beta^{\prime}=\left(\beta_{1}, \beta_{2}\right)^{\prime}, \beta_{1}$ é $k \times 1 \quad(k<p)$ e $\beta_{1}^{0}$ é um vetor especificado. Para testar $H_{0}$ contra a alternativa $\beta_{1} \neq \beta_{1}^{0}$, podemos usar a estatística da razão de verossimilhanças, 


$$
\Delta=-2 \log \left(\frac{L\left(\beta_{1}^{0}, \tilde{\beta}_{2}, \tilde{\sigma}\right)}{L\left(\hat{\boldsymbol{\beta}}_{1}, \hat{\boldsymbol{\beta}}_{2}, \hat{\sigma}\right)}\right),
$$

onde $\tilde{\beta}_{2}$ e $\tilde{\sigma}$ são os EMV de $\beta_{2}$ e $\sigma$ sob $H_{0}$ e $\hat{\sigma}$ e $\hat{\beta}=\left(\hat{\beta}_{1} \hat{\beta}_{2}\right)$ são os EMV de $\sigma$ e $\beta$ sob o modelo completo.

Da mesma forma que foi considerado o modelo de regressão exponencial, Weibull e o de valor extremo, podemos fazer inferências para modelos com outras distribuições, como por exemplo, a distribuição log-normal.

Análises Bayesianas do modelo (1.22) tem sido consideradas por vários autores, (ver por exemplo Achcar e Bolfarine, 1985).

\subsection{Objetivos}

Nesta dissertação, daremos ênfase às distribuições exponencial e a de Weibull para os dados, tempos de sobrevivência, sob o esquema de amostragem completa.

Um problema usual na análise Bayesiana desses modelos de regressão é encontrar densidades a posteriori marginais, que usualmente requer a determinação numérica de integrais. Uma alternativa seria o uso de. métodos de aproximações para integrais (ver por exemplo, Lindley, 1980; Tierney e Kadane,1986; Tierney, Kass e Kadane, 1989; Leonard, Hsu e Tsui, 1989). Apresentamos, no capítulo 2, alguns métodos usuais para integração em inferências Bayesianas, especialmente para calcular densidades a posteriori marginais e momentos a posteriori. 
É prática freqüente entre os estatísticos e pesquisadores fazer inferências sobre funções de parâmetros como por exemplo a função de sobrevivência, considerando a normalidade dos estimadores de máxima verossimilhança. Esses resultados podem não ser precisos quando trabalhamos com amostras pequenas ou moderadas. No capítulo 3 consideramos diferentes reparametrizações que melhoram a normalidade da função de verossimilhança (ver por exemplo Anscombe,1964 ou Sprott,1973). Também exploramos métodos gráficos que podem ser usados para verificar se uma parametrização proposta melhora a normalidade da densidade a posteriori ou da função de verossimilhança ( ver por exemplo Hills e Smith, 1993).

No capítulo 4 apresentamos análises clássica e Bayesiana do modelo de regressão de Feigl e Zelen (1965) assumindo uma distribuição de sobrevivência exponencial (1.7). A regra de Jeffreys (ver por exemplo, Box e Tiao, 1973) é usada para acharmos uma densidade a priori não-informativa para os parâmetros envolvidos.

No capítulo 5 apresentamos uma análise clássica do modelo de regressão locaçãoescala com a distribuição de valor extremo (1.23) assumindo uma distribuição de sobrevivência de Weibull (1.20). Também apresentamos técnicas de regressão na comparação de dois tratamentos usando'procedimentos de inferência clássica assim como de inferência Bayesiana.

Exemplos de aplicação das metodologias obtidas são apresentados no capítulo 6.

Finalmente no capítulo 7, são apresentadas conclusões e comentários sobre perspectivas futuras. 


\section{CAPÍTULO 2}

\section{ALGUNS MÉTODOS DE RESOLUÇÃO DE INTE- GRAIS EM INFERÊNCIA BAYESIANA.}

\subsection{Introdução}

Neste capítulo, apresentamos alguns métodos usuais para integração em inferência Bayesiana, especialmente para calcular características de densidades preditivas e posteriori marginais como suas médias e variâncias. Se o problema sob consideração não envolve densidade a priori conjugada, essa tarefa não pode ser realizada de forma fechada e métodos de aproximação analítica ou numérica são necessários. (ver por exemplo Naylor e Smith, 1982; Klock e Van Dijk, 1978; Zellner e Rossi, 1982; Lindley, 1980; Tierney e Kadane, 1986, Tierney, Kass e Kadane, 1989; Leonard, Hsu e Tsui, 1989; Casella e George, 1992).

Usualmente o estatístico Bayesiano tem interesse em encontrar momentos a posteriori para funções $g(\theta)$ da forma,

$$
\mathrm{E}(g(\theta) \mid D)=\frac{\int g(\theta) \pi(\theta) L(\theta \mid D) d \theta}{\int \pi(\theta) L(\theta \mid D) d \theta}
$$


onde $g(\theta)$ é uma função selecionada de $\theta \in R^{m}, \pi(\theta)$ é uma densidade a priori e $L(\theta \mid D)$ é a função de verossimilhança para $\theta$ dado o conjunto de dados $D$.

Da mesma forma, temos interesse em determinar densidades a posteriori marginais,

$$
\pi\left(\theta_{1} \mid D\right)=\int \pi\left(\theta_{1}, \theta_{2} \mid D\right) d \theta_{2}
$$

onde $\pi\left(\theta_{1}, \theta_{2} \mid D\right)$ é a densidade a posteriori conjunta de $\theta=\left(\theta_{1}, \theta_{2}\right), \quad \theta_{1} \in R^{k}$ e $\theta_{2} \in R^{m-k}$.

Na determinação de $E(g(\theta) \mid D)$ ou $\pi\left(\theta_{1} \mid D\right)$, podemos ter dificuldades na integração, que pode ser resolvida usando métodos numéricos ou de aproximação.

Outro problema de integração envolve a determinação de densidades preditivas. Sejam $X_{1}, \ldots X_{n}$ variáveis aleatórias com função densidade conhecida associada com os parâmetros $\theta_{1}, \ldots, \theta_{m}$ pertencente ao espaço paramétrico $\Theta$. A densidade preditiva para uma observação futura $X_{n+1}$ é dada por:

$$
f\left(x_{n+1} \mid x_{1}, \ldots, x_{n}\right)=\int_{\theta} f\left(x_{n+1} \mid \theta_{1}, \ldots, \theta_{m}\right) \pi\left(\theta_{1}, \ldots, \theta_{m} \mid x_{1}, \ldots, x_{n}\right) d \theta_{1}, \ldots d \theta_{m} .
$$

Assim, $f\left(x_{n+1} \mid x_{1}, \ldots, x_{n}\right)=E\left\{f\left(x_{n+1} \mid \theta_{1}, \ldots, \theta_{m}\right)\right\}$,onde $f\left(x_{n+1} \mid \theta_{1}, \ldots, \theta_{m}\right)$ é a função de densidade de $X_{n+1}$ e $\pi\left(\theta_{1}, \ldots, \theta_{m} \mid x_{1}, \ldots, x_{n}\right)$ é a densidade a posteriori dos parâmetros $\theta_{1}, \ldots, \theta_{m}$ (ver por exemplo Aitchison e Dunsmore, 1975). 


\subsection{Momentos a Posteriori}

\subsubsection{Aproximação de Tierney e Kadane}

Em problemas onde a densidade a posteriori conjunta é conhecida e há interesse em se fazer inferências a respeito de um parâmetro particular, o método introduzido por Tierney e Kadane (1986), baseado no método de aproximação de Laplace fornece resultados simples e com boa precisão.

O método de Laplace para aproximação de integrais é usado para resolver integrais da forma:

$$
I=\int f(\theta) \exp [-n h(\theta)] d \theta,
$$

onde $-n h(\theta)$ é uma função com um máximo $\hat{\theta}$ e satisfaz algumas condições usuais de regularidade.

Para aproximar integrais da forma (2.4), o método de Laplace assume um desenvolvimento de $h$ e $f$ em série de Taylor, em torno de $\hat{\theta}$ (ver Tierney e Kadane, 1986; Tierney, Kass e Kadane, 1989).

\section{Caso 1: $\theta$ Uniparamétrico}

$\operatorname{Com} \theta$ uniparamétrico, a aproximação de Laplace para $I$ é dada por : 


$$
\hat{I} \cong\left(\frac{2 \pi}{n}\right)^{1 / 2} \sigma f(\hat{\theta}) \exp \{-n h(\hat{\theta})\}, \text { onde } \sigma=\left\{h^{\prime \prime}(\hat{\theta})\right\}^{-1 / 2}
$$

Caso 2: $\theta \in R^{m} m$-dimensional

$\operatorname{Com} \theta \in R^{m}$, a aproximação de Laplace para $I$ é dada por:

$$
\hat{I} \cong(2 \pi)^{m / 2}\left\{\operatorname{det}\left(m D^{2} h(\hat{\theta})\right)\right\}^{-1 / 2} f(\hat{\theta}) \exp \{-n h(\hat{\theta})\}
$$

onde $D^{2} h(\hat{\theta})$ é a matriz Hessiana de $h$ calculada em $\hat{\theta}$ dada por :

$$
D^{2} h(\theta)=\left[\begin{array}{cccc}
\frac{\partial^{2} h}{\partial \theta_{1}^{2}} & \frac{\partial^{2} h}{\partial \theta_{1} \partial \theta_{2}} & \cdots & \frac{\partial^{2} h}{\partial \theta_{1} \partial \theta_{m}} \\
& \frac{\partial^{2} h}{\partial \theta_{2}^{2}} & \cdots & \frac{\partial^{2} h}{\partial \theta_{2} \partial \theta_{m}} \\
& & & \vdots \\
\text { simétrica } & & \ddots & \frac{\partial^{2} h}{\partial \theta_{m}^{2}}
\end{array}\right] .
$$

Para aproximar momentos a posteriori da forma (2.1) usando o método de Laplace, podemos considerar $\pi(\theta) L(\theta \mid D)=\exp \{-n h(\theta)\}$ para o numerador e denominador de (2.1), com $f$ igual a $g$ e 1 respectivamente. Assim, podemos ter uma aproximação modal da forma,

$$
\hat{E}\{g(\theta) \mid D\} \cong g(\hat{\theta})\left\{1+9\left(n^{-1}\right)\right\}
$$


onde $\hat{\theta}$ é a moda de $\pi(\theta \mid D)$ (ver por exemplo Tierney e Kadane,1986).

Podemos considerar outras escolhas para $f \mathrm{em}$ (2.4), nas integrais do numerador e denominador de (2.1.) para obtermos aproximações mais precisas para o momento a posteriori (2.1).

Considerando $f=1$ em (2.4) temos outra aproximação mais precisa, para $E\{g(\theta \mid D)\}$ em (2.1). Assim,

$$
E\{g(\theta) \mid D\}=\frac{\int e^{-n h^{*}(\theta)} d \theta}{\int e^{-n h(\theta)} d \theta}
$$

onde $g(\theta)$ é uma função positiva, $-n h(\theta)=\ln \pi(\theta)+\ln L(\theta \mid D) \quad$ e $-n h^{*}(\theta)=\ln g(\theta)-n h(\theta)$.

Usando o método de Laplace em (2.8) temos:

$$
\hat{E}\{g(\theta) \mid D\} \cong\left(\frac{\sigma^{*}}{\sigma}\right) \exp \left\{-\left[h^{*}\left(\hat{\theta}^{*}\right)-h(\hat{\theta})\right]\right\}
$$

onde $\hat{\theta}$ maximiza $-h(\theta), \quad \hat{\theta}^{*} \operatorname{maximiza}-h^{*}(\theta), \quad \sigma=\left\{\operatorname{det}\left(n D^{2} h(\hat{\theta})\right)\right\}^{-1 / 2} \mathrm{e}$ $\sigma^{*}=\left\{\operatorname{det}\left(n D^{2} h\left(\hat{\theta}^{*}\right)\right)\right\}^{-1 / 2}$. Esta aproximação satisfaz:

$$
E\{g(\theta) \mid D\}=\hat{E}\{g(\theta) \mid D\}\left(1+\vartheta\left(n^{-2}\right)\right) .
$$


Para uma amostra de tamanho $n$ grande, em (2.9), temos uma aproximação com grande precisão. Apesar disso, esta aproximação pode ser melhorada em muitos casos considerando uma reparametrização apropriada (ver por exemplo Achcar e Smith, 1990; ou Kass e Slate, 1992).

A parametrização escolhida é fundamental para uma boa precisão de Laplace, especialmente para pequenas amostras.

\subsubsection{Métodos Numéricos}

O uso de métodos numéricos é outra estratégia explorada por muitos estatísticos Bayesianos ( ver por exemplo Naylor e Smith, 1982). Usualmente, esses métodos são apropriados para problemas com número pequeno de parâmetros. Dos métodos utilizados podemos mencionar:

\section{a) Regra de Simpson}

Caso Uniparamétrico. O método de Simpson é dado por:

$$
\int_{a}^{b} f(\theta) d \theta=\frac{h}{3}\left(f_{0}+4 f_{1}+2 f_{2}+\ldots .+2 f_{2 n-2}+4 f_{2 n-1}+f_{2 n}\right),
$$

onde o intervalo finito $[a, b]$ é dividido em $2 n$ (número par) de subintervalos cada um de comprimento $h$, tal que $b-a=2 n h$. O erro de (2.11) é dado por: 


$$
R_{n}=-\frac{1}{180}(b-a) h^{\prime \prime} f^{(4)}(\xi), \quad \text { onde } \quad a<\xi<b
$$

A regra de Simpson é apropriada quando o intervalo $[a, b]$ é finito e $f(\theta)$ é diferenciável, sendo que isto pode não ser possível para muitas aplicações Bayesianas, ou $f(\theta)$ pode ser muito complicada.

\section{b) Regra Gaussiana}

Naylor e Smith (1982), exploram o uso de métodos de quadratura Gaussiana para calcular integrais Bayesianas.

Para usar a regra Gaussiana, devemos escolher uma função integrável não negativa $\omega(\theta)$, tal que,

$$
\int_{a}^{b} f(\theta) d \theta=\int_{a}^{b} g_{G}(\theta) \omega(\theta) d \theta
$$

onde $g_{G}(\theta)=f(\theta) / \omega(\theta)$ é uma função bem comportada.

Um resultado clássico em integração numérica é que se $P_{2 n-1}(\theta)$ denota um polinômio de grau $(2 n-1)$ em $\theta$ o qual é bem definido em $[a, b]$ e $\left\{f_{n}(\theta)\right\}$ denota a sequêencia de polinômios ortogonais em $[a, b]$, relativo a uma função de peso positivo $\omega(x)$, tal que $f_{n}(\theta)$ seja um polinômio positivo então,

$$
\int_{a}^{b} \omega(\theta) P_{2 n-1}(\theta) d \theta=\sum_{k=1}^{n} \alpha_{k} P_{2 n-1}\left(\theta_{k}\right)
$$


onde $\alpha_{k}$ são os coeficientes e os $\theta_{k s}^{\prime}$ são as raízes de $f_{n}(\theta)=0$. Os coeficientes são dados por:

$$
\alpha_{k}=\int_{a}^{b} \frac{\omega(\theta) f_{n}(\theta) d \theta}{\left(\theta-\theta_{k}\right) f_{n}\left(\theta_{k}\right)}
$$

Na prática, podemos usar diferentes sistemas de polinômios ortogonais. Uma possibilidade seria selecionarmos a classe de polinômios ortogonais de Hermite que são polinômios relativos à função de peso $\omega(\theta)=\exp \left(-\theta^{2}\right)$ no intervalo $[a, b]=[-\infty, \infty]$.

Nesse caso (2.13.) resulta na aproximação,

$$
\int_{-\infty}^{\infty} e^{-\theta^{2}} g_{G}(\theta) d \theta \cong \sum_{k=1}^{n} \alpha_{k} g_{G}\left(\theta_{k}\right)+R_{n}
$$

onde $\theta_{1}, \ldots, \theta_{n}$ são as raízes da equação polinomial de Hermite $H_{n}(\theta)=0$, e

$$
\alpha_{k}=\frac{2^{n-1} n ! \sqrt{\pi}}{n^{2}\left[H_{n-1}\left(\theta_{k}\right)\right]^{2}}
$$

Os valores das raízes $\theta_{k}$ e os coeficientes $\alpha_{k}$ são dados em tabelas (ver por exemplo Abramowitz e Stegun, 1965, p.924). Na tabela 1 temos alguns valores de $\theta_{k}$ e $\alpha_{k}$ para quadratura de Gauss-Hermite com $n \leq 5$. 
Tabela 1: Raiz da Equação Polinomial de Hermite $H_{n}(\theta)$ e Coeficiente para Quadratura de Gauss-Hermite com $n \leq 5$

\begin{tabular}{|lll|}
\hline$n$ & $\theta_{k}$ & $\alpha_{k}$ \\
\hline 1 & 0 & 1.772454 \\
3 & \pm 0.707107 & 0.886227 \\
& 0 & 1.181636 \\
4 & \pm 1.224745 & 0.295410 \\
& \pm 0.524648 & 0.804914 \\
5 & \pm 1.650680 & 0.081313 \\
& 0 & 0.945309 \\
& \pm 0.958572 & 0.393619 \\
& \pm 2.020183 & 0.019953 \\
\hline
\end{tabular}

\subsubsection{Procedimento de Monte Carlo}

Os métodos numéricos discutidos na seção (2.2.2) não são adequados para problemas de dimensão muito grande. Quando a ordem de integração (número de parâmetros) excede 5 ou 6 , uma maior precisão pode ser encontrada com integração de Monte Carlo (ver por exemplo Kloek e Van Dijk, 1978; ou Press, 1989).

Para o uso do procedimento de Monte Carlo de integrais para aproximar momentos a posteriori (2.1), devemos escolher uma densidade geradora $h^{*}(\theta)$ definida como uma Importance Density. A escolha da Importance Density $h^{*}(\theta)$, usualmente seguem dois pontos importantes:

1. $h^{*}(\theta)$ se aproxima da densidade a posteriori para $\theta$,

2. A amostra de tamanho M é facilmente gerada por $h^{*}(\theta)$. 
Suponha $\theta(k \times 1)$ o vetor de parâmetros de uma distribuição amostral e deseja-se calcular integrais da forma:

$$
I\left(x_{1}, \ldots, x_{n}\right)=\int g(\theta) \pi\left(\theta \mid x_{1}, \ldots, x_{n}\right) d \theta
$$

sendo $g(\theta)$ uma função de $\theta$.

Podemos rescrever, (2.16) por,

$$
I(D)=E_{\theta}[g(\theta) \mid D]
$$

onde $D$ denota os dados $\left(x, \ldots, x_{n}\right)$ e $E(\cdot)$ denota a esperança tomada sobre o espaço k-dimensional.

Seja $\left(\theta_{1}, \ldots, \theta_{M}\right) M$ pontos gerados independentemente da Importance Density $h^{*}(\theta)$. O momento a posteriori (2.1) pode ser rescrito como:

$$
\begin{aligned}
I(D) & =\frac{\int g(\theta) \pi(\theta) L(\theta \mid D) d \theta}{\int \pi(\theta) L(\theta \mid D) d \theta} \\
& =\int g(\theta) \omega(\theta) d \theta
\end{aligned}
$$

onde $\omega(\theta)=\frac{\pi(\theta) L(\theta \mid D)}{\int \pi(\theta) L(\theta \mid D) d \theta}$

Como uma aproximação para $I(\cdot)$ na equação (2.17), tomamos a média ponderada, 


$$
\hat{I}(D)=\sum_{m=1}^{M} \hat{\omega}\left(\theta_{m}\right) g\left(\theta_{m}\right)
$$

onde os pesos $\hat{\omega}(\theta)$ são dados por:

$$
\hat{\omega}\left(\theta_{m}\right)=\frac{\pi\left(\theta_{m}\right) L\left(\theta_{m} \mid D\right) / h^{*}\left(\theta_{m}\right)}{\sum_{m=1}^{M}\left[\pi\left(\theta_{m}\right) L\left(\theta_{m} \mid D\right) / h^{*}\left(\theta_{m}\right)\right]}
$$

Portanto, usando o método de Monte Carlo, encontramos uma aproximação para (2.1) dada por:

$$
\hat{E}[g(\theta) \mid D] \cong \sum_{m=1}^{M} \hat{\omega}\left(\theta_{m}\right) g\left(\theta_{m}\right)
$$

Observe que a soma dos pesos $\hat{\omega}\left(\theta_{m}\right)$ é igual à unidade. Também notamos que como $\pi\left(\theta_{m}\right)$ e $L\left(\theta_{m} \mid D\right)$ são dados em forma de razão, somente necessitamos de seus "Kernels" em lugar da densidade completa. A aproximação (2.18) depende da escolha de $h^{*}(\theta)$.

\subsection{Densidades a Posteriori Marginais}

Nesta seção, apresentaremos aproximações assintóticas de densidades marginais de funções não lineares $\eta=\boldsymbol{g}(\theta)$, onde podemos encontrar a densidade a posteriori marginal de $\eta$ baseada na especificação completa de uma transformação, ou podemos usar um dos procedimentos de aproximação para funções não lineares proposta por Tierney, Kass e Kadane (1989) e por Leonard, Hsu e Tsui (1989). 


\subsubsection{Aproximação de Tierney, Kass e Kadane (TKK)}

Quando consideramos a partição de $\theta \in R^{m}$ na forma $\theta=\left(\theta_{1}, \theta_{2}\right), \theta_{1} \in R^{k} \mathrm{e}$ $\theta_{2} \in R^{m-k}$, podemos usar o método de Laplace diretamente para obtermos aproximações para densidades a posteriori marginais de $\eta=g(\theta)=\theta_{1}$, dadas por:

$$
\pi\left(\theta_{1} \mid D\right)=\int \pi\left(\theta_{1}, \theta_{2} \mid D\right) d \theta_{2}
$$

onde $\pi\left(\theta_{1}, \theta_{2} \mid D\right)=c f\left(\theta_{1}, \theta_{2}\right) \exp \left\{-n h\left(\theta_{1}, \theta_{2}\right)\right\}$.

Usando o método de Laplace temos,

$$
\hat{\pi}_{1}\left(\theta_{1} \mid D\right) \cong(2 \pi)^{\frac{-k}{2}}\left\{\frac{\operatorname{det} \Psi\left(\theta_{1}\right)}{\operatorname{det} \Sigma}\right\}^{\frac{1}{2}} \frac{\pi\left(\theta_{1}, \hat{\theta}_{2}\left(\theta_{1}\right) \mid D\right)}{\pi\left(\hat{\theta}_{1}, \hat{\theta}_{2} \mid D\right)}
$$

onde $\mathcal{Z}$ é a inversa da matriz Hessiana de $n h\left(\theta_{1}, \theta_{2}\right)$ em $\hat{\theta}=\left(\hat{\theta}_{1}, \hat{\theta}_{2}\right)$, o máximo local de $-n h\left(\theta_{1}, \theta_{2}\right), \hat{\theta}_{2}\left(\theta_{1}\right)$ maximiza $-n h_{\theta_{1}}\left(\theta_{2}\right)=-n h\left(\theta_{1}, \theta_{2}\right)$ com $\theta_{1}$ fixo e $\mathbb{Z}\left(\theta_{1}\right)$ é a inversa da matriz Hessiana de $n h\left(\theta_{1}, \theta_{2}\right)$ em $\hat{\theta}_{2}\left(\theta_{1}\right)$. Geralmente consideramos $f\left(\theta_{1}, \theta_{2}\right)=1 \mathrm{em}(2.19)$.

Em muitas aplicações temos interesse em aproximar densidades a posteriori marginais de funções mais gerais $\eta=g(\theta), \theta \in R^{m}$, onde $g$ é uma função não linear suposta suave e ter o gradiente diferente de zero ou o jacobiano de posto $k$, na vizinhança da moda da densidade a posteriori para $\theta$. Em algumas aplicações, é possível usar a transformação global $\phi=\phi(\theta)$, tal que $\phi=\left(g(\theta), \theta_{2}\right)$, mas em muitos casos isso é difícil ou impossível de ser obtido. 
Tierney, Kass e Kadane (1989) propõe uma aproximação que não depende de uma especificação explícita de uma transformação. Considerando uma função $g(\theta)$ k-dimensional a aproximação TKK é dada por:

$$
\hat{\pi}_{I K K}(\eta \mid D) \cong(2 \pi)^{-K / 2}\left\{\frac{\operatorname{det} \mathcal{Z}(\eta)}{\operatorname{det}(\mathcal{Z}) \operatorname{det}\left[(D g)^{\prime} Z(\eta)(D g)\right]}\right\}^{1 / 2} \frac{\pi(\hat{\theta}(\eta) \mid D)}{\pi\left(\hat{\theta}_{1} \mid D\right)}
$$

onde $\mathcal{Z}$ é a inversa da matriz Hessiana de $n h(\theta)$ em $\hat{\theta}, \hat{\theta}(\eta)$ maximiza $-n h(\theta)$ sujeita à restrição $\eta=g(\theta), \Sigma(\eta)$ é a inversa da matriz Hessiana de $n h(\theta)$ em $\hat{\theta}(\eta)$ e $D g$ é o gradiente ou jacobiano de $g$ calculado em $\hat{\theta}(\eta)$.

Observe que como em (2.20) a constante normalizadora $c$ de (2.21) não necessita ser calculada porque é calculada no numerador e denominador de (2.21). Também note que se $g$ é k-dimensional, então $D g$ é um vetor coluna com componentes $\partial g\{\hat{\theta}(\eta)\} / \partial \theta_{i}$, para $i=1,2, \ldots, m$ e se $g$ é k-dimensional, então $D g$ é uma matriz $m \times k$ com elementos $\partial g_{j}\{\hat{\theta}(\eta)\} / \partial \theta_{i}$, para $i=1,2, \ldots, m$ e $j=1,2, \ldots, k$.

Na prática podemos escrever (2.21) na forma:

$$
\hat{\pi}_{T k k}(\eta \mid D) \propto\left\{\frac{\operatorname{det}(\mathcal{Z}(\eta))}{\operatorname{det}\left[(D g)^{\prime} \Sigma(\eta)(D g)\right]}\right\}^{1 / 2} \pi(\hat{\theta}(\eta)) .
$$




\subsubsection{Aproximação de Leonard, Hsu e Tsui (LHT)}

Leonard, Hsui e Tsui (1989) propõem outra forma de aproximar densidades a posteriori marginais para funções não lineares $g(\theta)$.

A aproximação LHT da densidade a posteriori marginal de $\eta=g(\theta)$ é considerada a partir do desenvolvimento do logaritmo da densidade a posteriori conjunta para $\theta$ em torno do máximo $\hat{\theta}(\eta)$ de $-n h(\theta)$, sujeita à restrição $\eta=g(\theta)$. A aproximação LHT é dada por:

$$
\hat{\pi}_{L H T}(\eta \mid D) \propto\{\operatorname{det} \mathbb{Z}(\eta)\}^{1 / 2} \pi(\hat{\theta}(\eta) \mid D) \exp \left\{\frac{1}{2} \ell_{\eta}^{\prime} \mathbb{Z}(\eta) \ell_{\eta}^{\prime}\right\} f(\eta \mid \hat{\theta}(\eta), \mathbb{Z}(\eta)),
$$

onde $\ell_{\eta}$ é o gradiente de $n h(\theta)$ em $\hat{\theta}(\eta), \tilde{\theta}(\eta)=\hat{\theta}(\eta)-\Sigma(\eta) \ell_{\eta}$ e $f(\eta \mid \mu, \mathbb{C})$ é a densidade de $\eta=g(\theta)$ em $\eta$ quando $\theta$ tem uma distribuição normal com média $\mu$ e matriz de covariância $\mathbb{C}$.

Leonard, Hsu e Tsui (1989) sugerem aproximar $f(\eta \mid \hat{\theta}(\eta), \mathcal{Z}(\eta))$ por uma distribuição normal com média $g(\tilde{\theta}(\eta))$ e variância $[D g(\hat{\theta}(\eta))]^{\prime} \mathcal{L}(\eta)[D g(\hat{\theta}(\eta))]$.

A derivação de (2.23) não assume que $g$ seja bem comportada. Em particular $g$ não precisa ser diferenciável na vizinhança de $\hat{\theta}$.

Se a função $g$ é diferenciável e o jacobiano é de posto completo próximo de $\hat{\theta}$, então o erro em usar a aproximação LHT será de $9\left(n^{-1}\right)$ numa vizinhança fixada de $\hat{\theta}$. Se $g$ é não linear poderia resultar no erro da ordem $\vartheta\left(n^{-1 / 2}\right)$ na vizinhança de $\hat{\theta}$. Por outro lado se $g$ não é suave e o jacobiano não é de posto completo próximo 
de $\hat{\theta}$ então, Leonard, Hsu e Tsui mostram que usando (2.23) como uma aproximação específica para $f$, podemos obter melhor resultado que (2.22).

\subsection{3 "Gibbs Sampler"}

O "Gibbs Sampler" é uma técnica para gerar a variável aleatória de uma distribuição marginal indiretamente sem ter que calcular sua densidade (ver por exemplo Casella e George, 1992). Essa técnica explora algumas propriedades das cadeias de Markov.

Consideraremos $\pi\left(\theta_{1}, \ldots, \theta_{n} \mid D\right)$ a densidade a posteriori conjunta para $\theta_{1}, \theta_{2}, \ldots, \theta_{n}$ e assumimos que temos interesse em encontrar alguma característica da densidade a posteriori marginal,

$$
\pi\left(\theta_{1} \mid D\right)=\int \ldots \int \pi\left(\theta_{1}, \ldots, \theta_{n} \mid D\right) d \theta_{2} \ldots d \theta_{n}
$$

como sua média a posteriori $E\left\{\theta_{1} \mid D\right\}$ ou sua variância a posteriori $\operatorname{Var}\left\{\theta_{1} \mid D\right\}$.

Quando calculamos ou aproximamos $\pi\left(\theta_{1} \mid D\right)$, o método de "Gibbs-Samples" gera uma amostra $\theta_{11}, \theta_{12}, \ldots, \theta_{1 n}$ de $\pi\left(\theta_{1} \mid D\right)$ onde podemos calcular sua média e sua variância, ou outras características de $\pi\left(\theta_{1} \mid D\right)$.

Para ilustrar a técnica de "Gibbs Sampler", primeiro exploramos o caso de duas variáveis. Iniciando com um par de variáveis aleatórias $\theta=\left(\theta_{1}, \theta_{2}\right), \theta \in R^{2}$. $O$ método de "Gibbs Sampler" gera uma amostra de $\pi\left(\theta_{1} \mid D\right)$ baseado nas distribuições 
condicionais $\pi\left(\theta_{1} \mid \theta_{2}, D\right)$ e $\pi\left(\theta_{2} \mid \theta_{1}, D\right)$, usualmente conhecidas. A seqüência de "Gibbs" de variáveis aleatórias é dada por:

$$
\theta_{20}^{\prime}, \theta_{10}^{\prime}, \theta_{21}^{\prime}, \theta_{11}^{\prime}, \theta_{22}^{\prime}, \theta_{12}^{\prime}, \ldots, \theta_{2 k}^{\prime}, \theta_{1 k}^{\prime}
$$

O valor inicial $\theta_{20}^{\prime}$ é especificado e os demais de (2.25) são obtidos iterativamente, por valores gerados por,

$$
\theta_{1 j}^{\prime} \sim \pi\left(\theta_{1} \mid \theta_{2 j}^{\prime}, D\right)
$$

e

$$
\theta_{2(j+1)}^{\prime} \sim \pi\left(\theta_{2} \mid \theta_{1 j}^{\prime}, D\right)
$$

A distribuição de $\theta_{1 k}$ converge para $\pi\left(\theta_{1} \mid D\right)$ quando $k \rightarrow \infty$. Uma prova simples desse resultado é dada por Casella e George (1992).

Gelfand e Smith (1990) propõem gerar $m$ seqüências de "Gibbs" independentes de comprimento $k$, e então usar o valor final de cada seqüência. Se $\mathbf{k}$ é suficientemente grande, temos uma amostra independente identicamente distribuída. aproximada de $\pi\left(\theta_{1} \mid D\right)$.

Nos próximos capítulos teremos aplicações dos métodos de resoluções de integrais apresentados neste capítulo, considerando alguns modelos particulares de regressão com dados de sobrevivência, como por exemplo o modelo de Feigl e Zelen. 


\section{CAPÍTULO 3}

\section{ALGUNS ASPECTOS DE REPARAMETRIZAÇÃO}

Neste capítulo mostramos o efeito de diferentes parametrizações na precisão dos resultados assintóticos baseados na "normalidade" da função de verossimilhança ou densidade a posteriori. $O$ desempenho de técnicas numéricas ou resultados assintóticos baseado na "normalidade" dos estimadores de máxima verossimilhança (ver por exemplo, Kass e Slate, 1992), podem não ser apropriados principalmente quando trabalhamos com amostras pequenas ou moderadas.

Uma alternativa para melhorar esses resultados, é considerar reparametrizaఢ̧ões apropriadas que melhorem a "normalidade" da função de verossimilhança ou densidade posteriori de interesse (ver por exemplo Anscombe, 1964 ou Sprott, 1973).

Também é apresentado um método gráfico proposto por Hills e Smith (1993) para verificar se a parametrização proposta é apropriada.

\subsection{Uma Reparametrização Útil}

Considere o caso uniparamétrico, onde queremos encontrar uma transformação um-a-um de $\theta$ para $\phi$ que melhore a normalidade da função de verossimi- 
Ihança. Uma possibilidade é procurarmos uma reparametrização $\phi$, tal que o valor esperado da derivada segunda do logaritmo da função de verossimilhança seja constante (ver Sprott, 1973). Com essa parametrização $\phi$, temos informação de Fisher constante, isto é, no contexto Bayesiano temos uma densidade a priori de Jeffreys localmente uniforme para $\phi$ (ver por exemplo, Box e Tiao, 1973).

Na parametrização original $\theta$, a densidade a priori não informativa de Jeffreys é dada por :

$$
\pi(\theta) \propto\{I(\theta)\}^{1 / 2}
$$

onde $I(\theta)$ é a informação de Fisher.

Com uma transformação um-a-um, temos:

$$
\pi(\theta) \propto \text { constante }\left|\frac{d \phi}{d \theta}\right|,
$$

onde podemos encontrar $\phi$ resolvendo a equação diferencial,

$$
\phi \propto \int\{I(\theta)\}^{1 / 2} d \theta
$$

(ver, Achcar, 1993). 
No contexto Bayesiano para o caso de dois parâmetros podemos procurar uma transformação um-a-um de $\left(\theta_{1}, \theta_{2}\right)$ para $\left(\phi_{1}, \phi_{2}\right)$, onde temos uma densidade a priori de Jeffreys localmente uniforme para $\theta_{1}$ e $\theta_{2}$ dada por:

$$
\pi\left(\theta_{1}, \theta_{2}\right) \propto\left\{\operatorname{det} I\left(\theta_{1}, \theta_{2}\right)\right\}^{1 / 2}
$$

e onde $I\left(\theta_{1}, \theta_{2}\right)$ é a matriz de informação de Fisher.

Da transformação um-a-um de $\left(\theta_{1}, \theta_{2}\right)$ para $\left(\phi_{1}, \phi_{2}\right)$ temos,

$$
\left\{\frac{\partial \phi_{1} \partial \phi_{2}}{\partial \theta_{1} \partial \theta_{2}}-\frac{\partial \phi_{1} \partial \phi_{2}}{\partial \theta_{1} \partial \theta_{2}}\right\} \propto \text { constante }\left\{\operatorname{det} I\left(\theta_{1}, \theta_{2}\right)\right\}^{1 / 2}
$$

com $E\left(-\partial^{2} \ell / \partial \phi_{1}^{2}\right)=$ constante, $E\left(-\partial^{2} \ell / \partial \phi_{2}^{2}\right)=$ constante e $E\left(-\partial^{2} \ell / \partial \phi_{1} \partial \phi_{2}\right)=$ constante, onde $\ell$ é o logaritmo da função de verossimilhança para $\phi_{1}$ e $\phi_{2}$.

Uma aplicação deste resultado é dado por Achcar (1991), considerando uma reparametrização para os parâmetros da distribuição de valor extremo.

Em geral temos grandes dificuldades para resolver a equação diferencial (3.5) para encontrarmos uma reparametrização apropriada. Em trabalhos práticos, usualmente consideramos diferentes parametrizações e usando algumas medidas diagnóstico para não-normalidade, como propostas por Kass e Slate (1992), podemos decidir pela melhor parametrização como será considerado neste trabalho, onde o parâmetro de interesse será dado pela função de sobrevivência num dado tempo. 


\subsection{Uma Medida Diagnóstica de Não-Normalidade}

Um caminho para verificarmos a "normalidade" da função de verossimilhança, ou da densidade a posteriori para parâmetros de interesse, é através de gráficos e contornos no caso bivariado. Isto é, concluímos pela "normalidade" avaliando a simetria e elipsidade respectivamente. No caso quando temos interesse num dado parâmetro e existem vários parâmetros auxiliares (nuisance parameters), podemos considerar a verossimilhança "profile" e verificar sua "normalidade" através da simetria dos gráficos.

Kass e Slate (1992) propõem algumas medidas diagnóstico de nãonormalidade da densidade a posteriori ou função de verosimilhança. Entre essas medidas, destacamos a "derivada terceira padronizada", que foi originalmente proposta por Sprott, 1973, e que reduz as análises a um simples escalar.

\subsubsection{Derivada Terceira Padronizada (STD)}

\section{i) Caso Uniparamétrico}

Para verificarmos a não-normalidade da função de verossimilhança na parametrização $\theta$, consideramos a derivada terceira da função de verossimilhança na forma padronizada dada por: 


$$
S T D=\left|\ell^{\prime \prime \prime}(\hat{\theta})\left[-\ell^{\prime \prime}(\hat{\theta})\right]^{-3 / 2}\right|
$$

onde $\ell^{\prime \prime}(\hat{\theta})$ e $\ell^{\prime \prime \prime}(\hat{\theta})$ são, respectivamente, a segunda e terceira derivadas do logaritmo da função de verossimilhança no estimador de máxima verossimilhança $\hat{\theta}$ (ver por exemplo Kass e Slate, 1992; Sprott, 1973 ou Achcar, 1993).

Se a função de verossimilhança apresentar boa "normalidade", esperamos encontrar $\operatorname{STD}(\hat{\theta})$, dado em (3.7), aproximadamente ou igual a zero.

De forma similar podemos calcular a derivada terceira padronizada da densidade a posteriori substituindo $\hat{\theta}$ por $\tilde{\theta}$, onde $\tilde{\theta}$ é a moda da densidade a posteriori.

A importância da derivada terceira padronizada dada por $S T D(\hat{\theta})$, em (3.7) é que ela é invariante para transformações afins dos parâmetros.

\section{ii) Caso Multiparamétrico}

No caso de dois parâmetros, podemos verificar a "normalidade" da função de verossimilhança ou densidade a posteriori com auxílio de gráficos de contorno. Em situações com três ou mais parâmetros podemos usar uma medida sumária das derivadas terceiras para diagnosticar a "normalidade" conjunta (ver Kass e Slate 1992) dada por:

$$
m^{2} \bar{B}^{2}=\sum_{i, j, k, j, m, n} b_{i j} b_{l m} b_{k n} d_{i j k} d_{l m n}
$$


onde $b_{i j}$ são os elementos da inversa da matriz de informação, $d_{i j k}=\partial^{3} \ell(\hat{\theta}) / \partial \theta_{i} \partial \theta_{j} \partial \theta_{k}$, para um parâmetro $\theta$ m-dimensional e o somatório sobre todos os índices. Observe que essa medida reduz-se para o quadrado da derivada terceira padronizada no caso uniparamétrico dada em (3.7).

No mesmo caminho podemos usar (3.8) para verificar a "normalidade" da densidade a posteriori substituindo $\hat{\theta}$ por $\tilde{\theta}$, a moda da densidade a posteriori.

Neste trabalho, utilizaremos a derivada terceira padronizada do logaritmo da função de verossimilhança para verificar a "normalidade" para as reparametrizações consideradas.

\subsection{Reparametrização de Guerrero e Johnson para a Função de Sobrevivência num Dado Tempo t}

Se o parâmetro de interesse é dado pela função de sobrevivência $R(t)$ num dado tempo $t$, algumas famílias de transformações paramétricas para proporções (ver por exemplo Atkinson, 1985, ou Achcar, 1992) podem ser exploradas para melhorar a "normalidade" da verossimilhança de $R(t)$. Nesta seção exploramos uma reparametrização introduzida por Guerrero e Johnson (1982).

Para obtermos uma família inversivel de transformações a qual inclui a transformação logito, Guerrero e Johnson (1982) sugeriram uma aplicação da transformação potência de Box e Cox (1964) para a "odds ratio", $R /(1-R)$, dada por: 


$$
\phi_{G J}^{*}(\lambda)=\left\{\left(\frac{R}{1-R}\right)^{\lambda}-1\right\} / \lambda, \quad-1 \leq \phi_{G J}(\lambda)<\infty
$$

onde $R=R(t)$.

Para um dado $\lambda$, podemos considerar uma forma modificada da transformação de Guerrero e Johnson dada por:

$$
\phi_{G J}(\lambda)=\left(\frac{R}{1-R}\right)^{\lambda}-1
$$

que não deve produzir resultados diferentes como considerado em (3.9).

A vantagem da transformação (3.10) é ser facilmente inversível. Com $\phi_{G J}=\phi_{G J}(\lambda)$, obtemos,

$$
R=\frac{\left(\phi_{G J}+1\right)^{1 / \lambda}}{1+\left(\phi_{G J}+1\right)^{1 / \lambda}}
$$

Desta forma, a transformação pode ser facilmente usada para obtermos estimativas e intervalos de confiança para os dados na escala original. 


\subsection{Diagnóstico Gráfico para Verificar uma}

\section{Parametrização}

Como já foi citado anteriormente ao verificarmos a "normalidade" da função de verossimilhança analisamos a assimetria ou a elipsidade dos gráficos, não tendo como medir o grau de "normalidade", ou seja, o quanto estamos próximos, ou não, da "normalidade" desejada.

Um outro método gráfico que podemos usar para verificarmos se uma parametrização proposta é adequada, ou seja, se existe "normalidade" da função de verossimilhança ou densidade a posteriori na parametrização proposta é dado pelo "tplot" proposto por Hills e Smith (1993), que verifica a "normalidade" através da linearidade do gráfico.

O diagnóstico proposto é dado por:

$$
T^{*}\left(\theta_{j}\right)=\operatorname{sgn}\left(\theta_{j}-\hat{\theta}_{j}\right)\left[-2 \log \left\{\frac{p\left(\theta_{j} \mid x\right)}{p(\hat{\theta} \mid x)}\right]^{1 / 2},\right.
$$

onde $\hat{\theta}_{j}$ é a moda da densidade a posteriori $p\left(\theta_{j} \mid x\right)$ e $\operatorname{sgn}\left(\theta_{j}-\hat{\theta}_{j}\right)$ é a função sinal. 0 gráfico resultante de $T^{*}\left(\theta_{j}\right)$ contra $\theta_{j}$ será uma linha reta se a distribuição a posteriori para $\theta_{j}$ é de fato normal. Conseqüentemente o gráfico pode ser usado para identificar desvios da "normalidade".

No caso unidimensional o t-plot de Hills e Smith é definido (de 3.12) por, 


$$
T(\theta)=\operatorname{sgn}(\theta-\hat{\theta})\left\{-2 \log \left[\frac{p(\theta \mid x)}{p(\hat{\theta} \mid x)}\right]\right\}^{1 / 2}
$$

No caso bidimensional a extensão de (3.12) apresenta dificuldades, mas fazendo a expansão em série de Taylor de $\log p\left(\theta_{1}, \theta_{2} \mid x\right)$ em torno de $\hat{\theta}=\left(\hat{\theta}_{1}, \hat{\theta}_{2}\right)$ e procedendo como no caso uni-dimensional, um gráfico de contorno de $T^{*}$ contra $\theta_{1}$ e $\theta_{2}$ pode ser construído. Se $\theta_{1}$ e $\theta_{2}$ têm distribuição aproximadamente normal, então o contorno deve ter a forma de uma elipse.

Em nosso trabalho usaremos o "t-plot" de Hills e Smith para avaliar a "normalidade" da função de verossimilhança $L(\theta)$, a partir da seguinte relação:

$$
T^{*}(\theta)=\operatorname{sgn}(\theta-\hat{\theta})\left\{-2 \log \left[\frac{L(\theta)}{L(\hat{\theta})}\right]\right\}^{1 / 2}
$$

onde $\hat{\theta}$ é o estimador de máxima verossimilhança de $\theta$. 


\section{CAPÍTULO 4}

\section{MODELO DE REGRESSÃO DE FEIGL E ZELEN}

Neste capitulo será apresentada uma análise clássica e uma análise Bayesiana para os parâmetros do modelo de regressão de Feigl e Zelen, considerando dados de uma distribuição exponencial. Em particular, consideramos inferências sobre a função de sobrevivência num dado tempo $t$.

\subsection{Análise Clássica do Modelo}

Nesta seção, será apresentada uma análise clássica para determinação de estimadores de máxima verossimilhança e intervalos de confiança para os parâmetros do modelo de regressão de Feigl e Zelen (1965), considerando que os dados provêm de uma amostra com distribuição exponencial. Através desses estimadores, serão feitas inferências sobre a função de sobrevivência $R(t)$. Para obtermos melhores resultados assintóticos, exploramos o uso de diferentes reparametrizações. 


\subsubsection{Formulação do Modelo}

Seja $T$ uma variável aleatória não-negativa denotando o tempo de sobrevivência suposto ter uma distribuição exponencial com função densidade de probabilidade (f.d.p.) dada por:

$$
f(t, \lambda)=\frac{1}{\lambda} \exp \left\{-\frac{t}{\lambda}\right\}
$$

onde, $\lambda>0$ é um parâmetro desconhecido representando a taxa constante de falha.

O tempo médio de sobrevivência é dado por:

$$
E(T)=\lambda
$$

Seja $\lambda=\theta_{1} e^{\theta_{2} x}$ e $x$ é uma covariável associada à $t$. Assim,

$$
f\left(t ; \theta_{1}, \theta_{2}\right)=\frac{1}{\theta_{1} e^{\theta_{2} x}} \exp \left\{-\frac{t}{\theta_{1} e^{\theta_{2} x}}\right\} \quad t>0
$$

é o modelo proposto por Feigl e Zelen (1965) (ver capítulo 1).

A função de sobrevivência num dado tempo $t_{0}$ é dada por:

$$
R\left(t_{0}\right)=P\left[T>t_{0}\right]=\exp \left\{-\frac{t_{0}}{\theta_{1} e^{\theta_{2} x_{0}}}\right\}
$$


onde $t_{0}$ é fixo e $x_{0}$ é o valor da covariável fixada.

Temos agora um modelo estatístico e a relação entre o parâmetro do modelo e a covariável. Torna-se possível então estimarmos os parâmetros da distribuição que geram os dados e os parâmetros de interesse.

O método de máxima verossimilhança permite estimar os parâmetros $\theta_{1}$ e $\theta_{2}$, e conseqüentemente estimar a função de sobrevivência $R$. Este método consiste em encontrar o valor dos parâmetros $\theta_{1}$ e $\theta_{2}$ que, com maior probabilidade, produzem os dados.

\subsubsection{Aproximação Normal para os Estimadores de Máxima Verossimilhança}

É prática freqüente entre os estatísticos e pesquisadores fazer inferências sobre parâmetros ou funções de parâmetros, como por exemplo a função de sobrevivência num dado tempo $t$. Geralmente nessas situações, não é possível obtermos resultados exatos, sendo comum considerarmos a "normalidade" assintótica de estimador de máxima verossimilhança, baseada na informação de Fisher ou informação observada (ver por exemplo Bickel e Doksum, 1977).

A função de verossimilhança dos parâmetros sob o modelo (4.2) com dados sem censuras é dada por:

$$
L\left(\theta_{1}, \theta_{2}\right)=\prod_{i=1}^{n} \theta_{1}^{-1} e^{-\theta_{2} x_{i}} \exp \left\{-\frac{1}{\theta_{1}} t_{i} e^{-\theta_{2} x_{i}}\right\}
$$


Isto é,

$$
L\left(\theta_{1}, \theta_{2}\right)=\theta_{1}^{-n} e^{-n \pi \theta_{2}} \exp \left\{-\frac{1}{\theta_{1}} \sum_{i=1}^{n} t_{i} e^{-\theta_{2} x_{i}}\right\} .
$$

Suponhamos que exista um valor $\left(\hat{\theta}_{1}, \hat{\theta}_{2}\right)$ que maximize $L\left(\theta_{1}, \theta_{2}\right)$. Então $\left(\hat{\theta}_{1}, \hat{\theta}_{2}\right)$ é chamado de estimador de máxima verossimilhança de $\left(\theta_{1}, \theta_{2}\right)$ (ver por exemplo, Bickel e Doksum, 1977).

O logaritmo da função de verossimilhança (4.4) é dado por:

$$
\ell\left(\theta_{1}, \theta_{2}\right)=-n \ln \theta_{1}-n \bar{x} \theta_{2}-\frac{1}{\theta_{1}} \sum_{i=1}^{n} t_{i} e^{-\theta_{2} x_{i}}
$$

Os estimadores de máxima verossimilhança $\left(\hat{\theta}_{1}, \hat{\theta}_{2}\right)$ obtidos de $\partial \ell\left(\theta_{1}, \theta_{2}\right) / \partial \theta_{1}=0$ e $\partial \ell\left(\theta_{1}, \theta_{2}\right) / \partial \theta_{2}=0$, são soluções das equações,

$$
\frac{-n}{\theta_{1}}+\frac{1}{\theta_{1}^{2}} \sum_{i=1}^{n} t_{i} e^{-\theta_{2} x_{i}}=0
$$

e

$$
-n \bar{x}+\frac{1}{\theta_{1}} \sum_{i=1}^{n} t_{i} x_{i} e^{-\theta_{2} x_{i}}=0, \text { respectivamente. }
$$

Assim, $\hat{\theta}_{1}$ e $\hat{\theta}_{2}$ são obtidos por, 


$$
\left\{\begin{array}{l}
\hat{\theta}_{1}=\left(\sum_{i=1}^{n} t_{i} e^{-\hat{\theta}_{2} x_{i}}\right) / n \\
\bar{x} \sum_{i=1}^{n} t_{i} e^{-\hat{\theta}_{2} x_{i}}=\sum_{i=1}^{n} t_{i} x_{i} e^{-\hat{\theta}_{2} x_{i}} .
\end{array}\right.
$$

Para solucionar numericamente a equação em relação a $\hat{\theta}_{2}$ podemos recorrer a um método iterativo, como por exemplo o método iterativo de Newton-Raphson.

Usando a teoria assintótica para grandes amostras, testes de hipóteses ou intervalos de confiança podem ser obtidos para os parâmetros a partir dos estimadores de máxima verossimilhança $\hat{\theta}_{1}$ e $\hat{\theta}_{2}$.

Suponhamos que a função de verossimilhança $L\left(\theta_{1}, \theta_{2}\right)$, de uma amostra aleatória de tamanho $n$, seja diferenciável em $\theta_{1}$ e $\theta_{2}$ até a segunda ordem e se $\left(\hat{\theta}_{1}, \hat{\theta}_{2}\right)$ são estimadores de máxima verossimilhança para $\left(\theta_{1}, \theta_{2}\right)$ então $\left(\hat{\theta}_{1}, \hat{\theta}_{2}\right)$ têm distribuição Normal assintótica dada por:

$$
\left(\hat{\theta}_{1}, \hat{\theta}_{2}\right) \stackrel{a}{\sim} N\left\{\left(\theta_{1}, \theta_{2}\right) ; I^{-1}\left(\hat{\theta}_{1}, \hat{\theta}_{2}\right)\right\}
$$

onde $I\left(\theta_{1}, \theta_{2}\right)$ é a matriz de informação de Fisher, dada por:

$$
I\left(\theta_{1}, \theta_{2}\right)=\left(E\left(-\frac{\partial^{2} \ell}{\partial \theta_{1}^{2}}\right) \quad E\left(-\frac{\partial^{2} \ell}{\partial \theta_{1} \partial \theta_{2}}\right)\right) .
$$


Notemos que $I^{-1}\left(\hat{\theta}_{1}, \hat{\theta}_{2}\right)$, o inverso da matriz de informação de Fisher, é a matriz de variância e covariância assintótica de $\hat{\theta}_{1}$ e $\hat{\theta}_{2}$, dada por:

$$
I^{-1}\left(\hat{\theta}_{1}, \hat{\theta}_{2}\right)=\left(\begin{array}{cc}
\hat{\sigma}_{\theta_{1}}^{2} & \hat{\sigma}_{\theta_{1} \theta_{2}} \\
\hat{\sigma}_{\theta_{2} \theta_{1}} & \hat{\sigma}_{\theta_{2}}^{2}
\end{array}\right),
$$

(ver por exemplo, Bickel e Doksum, 1977).

A partir da função de verossimilhança dada em (4.4) e notando que $E\left(t_{i}\right)=\theta_{1} e^{\theta_{2} x_{i}}$, temos,

$$
\begin{aligned}
& E\left(-\frac{\partial^{2} \ell}{\partial \theta_{1}^{2}}\right)=\frac{n}{\theta_{1}^{2}} \\
& E\left(-\frac{\partial^{2} \ell}{\partial \theta_{2}^{2}}\right)=\sum_{i=1}^{n} x_{i}^{2} \mathrm{e} \\
& E\left(-\frac{\partial^{2} \ell}{\partial \theta_{1} \partial \theta_{2}}\right)=\frac{1}{\theta_{1}} \sum_{I=1}^{n} x_{i} .
\end{aligned}
$$

Portanto a matriz de informação de Fisher é dada por:

$$
I\left(\theta_{1}, \theta_{2}\right)=\left(\begin{array}{cc}
n / \theta_{1}^{2} & \sum x_{i} / \theta_{1} \\
\sum x_{i} / \theta_{1} & \sum x_{i}^{2}
\end{array}\right)
$$


Intervalos de confiança para os parâmetros $\theta_{1}$ e $\theta_{2}$, considerando um nível de confiança $100(1-\alpha) \%$ são dados por:

$$
\begin{aligned}
& P\left[\hat{\theta}_{1}-z_{\alpha / 2} \hat{\sigma}_{\theta_{1}} \leq \theta_{1} \leq \hat{\theta}_{1}+z_{\alpha / 2} \hat{\sigma}_{\theta 1}\right]=1-\alpha \\
& P\left[\hat{\theta}_{2}-z_{\alpha / 2} \hat{\sigma}_{\theta_{2}} \leq \theta_{2} \leq \hat{\theta}_{2}+z_{\alpha / 2} \hat{\sigma}_{\theta_{2}}\right]=1-\alpha,
\end{aligned}
$$

onde $z_{\alpha / 2}$ é um percentil da distribuição normal padronizada.

Para inferências pontuais sobre a função de sobrevivência, usamos a propriedade da invariância dos estimadores de máxima verossimilhança.

Notemos que o estimador de máxima verossimilhança para a função de sobrevivência dada em (4.2), pela propriedade de invariância dos estimadores de máxima verossimilhança é dado por:

$$
\hat{R}\left(t_{0}\right)=\exp \left\{-\frac{t_{0}}{\hat{\theta}_{1} e^{\hat{\theta}_{2} x_{0}}}\right\}
$$

Considere a transformação $\left(\theta_{1}, \theta_{2}\right)$ em $\left(R, \phi_{2}\right)$, onde $R=R\left(t_{0}\right)$ num dado valor $x_{0}$ da covariável, dada por:

$$
\left\{\begin{array}{l}
R=\exp \left\{-\frac{t_{0}}{\theta_{1} e^{\theta_{2} x_{0}}}\right\} \\
\phi_{2}=\theta_{2}
\end{array}\right\}
$$


O logaritmo da função de verossimilhança em termos de $R=R\left(t_{0}\right)$ é dado por:

$$
\ell\left(R, \phi_{2}\right)=-n \ln t_{0}+n \ln [(-\ln R)]+n \phi_{2}\left(x_{0}-\bar{x}\right)+\frac{\ln R}{t_{0}} \sum_{i=1}^{n} t_{i} e^{-\phi_{2}\left(x_{i}-x_{0}\right)}
$$

Portanto, os estimadores de máxima verossimilhança para $\left(R, \phi_{2}\right)$, são soluções das equações:

$$
\left\{\begin{array}{l}
\frac{\partial \ell}{\partial R}=\frac{n}{R \ln R}+\frac{1}{R t_{0}} \sum_{i=1}^{n} t_{i} e^{-\phi_{2}\left(x_{i}-x_{0}\right)} \\
\frac{\partial \ell}{\partial \phi_{2}}=-n\left(x_{0}+\bar{x}\right)+\frac{(-\ln R)}{t_{0}} \sum_{i=1}^{n} t_{i}\left(x_{i}-x_{0}\right) e^{-\phi_{2}\left(x_{i}-x_{0}\right)}
\end{array}\right.
$$

A equação (4.14) é não linear. Para solucionar numericamente esta equação podemos recorrer a métodos iterativos, como por exemplo o método iterativo de Newton-Raphson.

Os estimadores de máxima verossimilhança determinados $\left(\hat{R}, \hat{\phi}_{2}\right)$, têm uma distribuição normal assintótica dada por:

$$
\left(\hat{R}, \hat{\phi}_{2}\right) \stackrel{a}{\sim} N\left\{\left(R, \phi_{2}\right) ; I_{0}^{-1}\left(\hat{R}, \hat{\phi}_{2}\right)\right\}
$$

Em geral o pesquisador deve tomar muito cuidado no uso desses resultados assintóticos, especialmente para amostras pequenas ou moderadas, pois a aproxima- 
ção pode ser muito ruim comprometendo a precisão das estimativas. Uma alternativa então seria obtermos uma reparametrização que forneça boa "normalidade" para a função de verossimilhança. Em nosso trabalho exploramos a reparametrização de Guerrero e Johnson (ver capítulo 3).

Considerando $\phi_{2}$ desconhecido podemos calcular a verossimilhança "profile" $\ell_{\hat{\ell}_{2}}(R)$ para cada valor de $R$, onde o valor de $R$ que maximiza $\ell_{\hat{\phi}_{2}}(R)$ é dado por:

$$
\hat{R}=\exp \left[-n t_{0} / \sum_{i=1}^{n} t_{i} e^{-\hat{\phi}_{2}\left(x_{i}-x_{0}\right)}\right]
$$

Observar que podemos usar a "normalidade" assintótica de $\hat{R}$ para construir testes de hipóteses e intervalos de confiança para $R$.

Para avaliar a "normalidade" da função de verossimilhança podemos recorrer à derivada terceira padronizada do logaritmo da função de verossimilhança, localmente na estimativa de máxima verossimilhança ou outros métodos descritos no capítulo 3.

\subsubsection{Reparametrização de Guerrero e Johnson para a Função de Sobrevivência}

Os resultados assintóticos mencionados anteriormente podem não ser apropriados quando trabalhamos com amostras pequenas ou moderadas, principalmente quando estamos interessados numa sobrevivência muito baixa ou muito alta. Uma 
alternativa para melhorar estes resultados, é considerar reparametrizações que melhorem a "normalidade" da função verossimilhança (ver por exemplo Anscombe, 1964 ou Sprott, 1973).

Nesta subseção exploramos uma reparametrização introduzida por Guerrero e Johnson (1982), onde temos a seguinte transformação dada em (3.10):

$$
\left\{\begin{array}{l}
\phi_{G J}(\lambda)=\left(\frac{R}{1-R}\right)^{\lambda}-1 \\
e \\
\phi_{2}=\phi_{2},
\end{array}\right.
$$

O logaritmo da função de verossimilhança para $\phi_{G J}$ e $\phi_{2}$ é dado por:

$$
\ell\left(\phi_{G J}, \phi_{2}\right)=-n \ln t_{0}+\ln B\left(\phi_{G J}\right)+n \phi_{2}\left(x_{0}-\bar{x}\right)-\frac{B\left(\phi_{G J}\right)}{t_{0}} \sum_{i=1}^{n} t_{i} e^{-\phi_{2}\left(x_{i}-x_{0}\right)}
$$

onde, $A\left(\phi_{G J}\right)=\left(\phi_{G J}+1\right)^{-1 / \lambda}$ e $B\left(\phi_{G J}\right)=\ln \left[1+A\left(\phi_{G J}\right)\right]$.

Considerando $\phi_{2}$ desconhecido podemos calcular a verossimilhança "profile" $\ell_{\hat{\phi}_{2}}\left(\phi_{G J}\right)$ para cada valor de $\phi_{G J}$, onde podemos encontrar o valor de $\phi_{G J}$ que maximiza $\ell_{\hat{\phi}_{2}}\left(\phi_{G J}\right)$ resolvendo a seguinte equação:

$$
\frac{n}{B\left(\phi_{G J}\right)}=\frac{\sum_{i=1}^{n} t_{i} e^{-\hat{\phi}_{2}\left(x_{i}-x_{0}\right)}}{t_{0}}
$$


Podemos fazer 0 gráfico de $\ell_{\hat{\phi}_{2}}\left(\phi_{G J}\right)$ versus $\phi_{G J}$ e verificar a "normalidade" da verossimilhança "profile" $\ell_{\hat{\phi}_{2}}\left(\phi_{G J}\right)$ através da figura obtida.

Para determinarmos qual é o valor mais apropriado de $\lambda$ que forneça boa normalidade para a função de verossimilhança, utilizamos a derivada terceira padronizada do logaritmo da verossimilhança definida em (3.7).

A derivada terceira padronizada (STD) do logaritmo da função de verossimilhança localmente em $\hat{\phi}_{G J}$, é dada por:

$$
S T D\left(\hat{\phi}_{G J}\right)=\left|n^{-1 / 2}\left(2-\frac{3 B\left(\hat{\phi}_{G J}\right) B^{\prime \prime}\left(\hat{\phi}_{G J}\right)}{\left(B^{\prime}\left(\hat{\phi}_{G J}\right)\right)^{2}}\right)\right|
$$

Assim, o valor de $\lambda$ tal que $\operatorname{STD}\left(\hat{\phi}_{G J}\right) \cong 0$, pode ser encontrado através de um gráfico de $g(\lambda)=B\left(\hat{\phi}_{G J}\right) B^{\prime \prime}\left(\hat{\phi}_{G J}\right) /\left[B^{\prime}\left(\hat{\phi}_{G J}\right)\right]^{2}-2 / 3$ versus $\lambda$.

Com $\lambda$ obtido podemos considerar a "normalidade" assintótica de $\hat{\phi}_{G J}$ e conseguir melhores inferências para a função de sobrevivência $R$, especialmente para amostras pequenas ou moderadas.

\subsection{Análise Bayesiana do Modelo}

Nesta seção será apresentada uma análise Bayesiana para o modelo de regressão de Feigl e Zelen (1965). Considerando uma densidade a priori não-informativa de Jeffreys, será obtida a densidade a posteriori para os parâmetros $\theta_{1}$ e $\theta_{2}$. Conside- 
rando inicialmente $\theta_{1}$ e $\theta_{2}$ parâmetros desconhecidos, obtemos a densidade a posteriori conjunta para esses parâmetros e utilizando métodos de aproximações e métodos numéricos para integrais descritas no capitulo 3 , encontramos a densidade a posteriori para o parâmetro de interêsse $R$. Também exploramos a reparametrização de Guerrero e Johnson para melhorar a normalidade da densidade a posteriori para $R$.

Em análise de sobrevivência quando assumimos um modelo paramétrico para o tempo de vida de pacientes, temos interesse em estimar o valor do parâmetro que o define. Embora este parâmetro seja considerado fixo, não conhecemos o seu verdadeiro valor . Uma boa maneira de expressar nossa incerteza antes de relacionar uma amostra é através de uma distribuição de probabilidade a priori para o parâmetro, que sob este ponto de vista é considerado uma variável aleatória.

O teorema de Bayes leva à determinação da densidade de probabilidade a posteriori do parâmetro, incorporando a informação dos dados observados, com a informação adicional dada pela densidade a priori (ver Box e Tiao, 1973).

\subsubsection{Uma Densidade a Priori Não-Informativa para $\theta_{1}$ e $\theta_{2}$}

Antes de analisar os dados podemos incorporar conhecimento prévio a respeito dos parâmetros do modelo. Uma forma de se fazer isto é em termos de uma densidade de probabilidade para o parâmetro. Essa densidade de probabilidade é identificada como densidade a priori. Quando não temos nenhuma informação a respeito dos parâmetros devemos formular uma priori tal que toda informação venha exclusivamente dos dados, ou seja, devemos considerar uma priori não-informativa. Para 
isso, existem várias regras para se formular uma priori não-informativa; neste trabalho destacamos o uso da priori não- informativa de Jeffreys.

A escolha da priori não-informativa de Jeffreys é justificada por suas boas propriedades (ver Box e Tiao, 1973).

Assumindo que o pesquisador não tenha informação a respeito dos parâmetros $\theta_{1}$ e $\theta_{2}$, a regra de Jeffreys determina uma densidade a priori, não informativa,dada por:

$$
\pi\left(\theta_{1}, \theta_{2}\right) \propto\left\{\operatorname{det} I\left(\theta_{1}, \theta_{2}\right)\right\}^{1 / 2},
$$

onde $I\left(\theta_{1}, \theta_{2}\right)$ é a matriz de informação de Fisher (ver, Box e Tiao, 1973).

Considerando o modelo de Feigl e Zelen dado em (4.2), a densidade a priori de Jeffreys para $\theta_{1}$ e $\theta_{2}$ é dada por:

$$
\pi\left(\theta_{1}, \theta_{2}\right) \propto \frac{1}{\theta_{1}} \quad \theta_{1} \text { e } \theta_{2} \in \Theta
$$

onde $\Theta=\left\{\left(\theta_{1}, \theta_{2}\right) ; \theta_{1}>0,-\infty<\theta_{2}<\infty\right\}$. 


\subsubsection{Densidade a Posteriori Conjunta para $\theta_{1}$ e $\theta_{2}$}

Uma vez definido o modelo para os dados e a distribuição a priori, devemos combinar a informação prévia sobre o parâmetro com a informação contida nos dados, obtendo assim uma densidade a posteriori.

Com a priori (4.12), a densidade a posteriori conjunta para $\theta_{1}$ e $\theta_{2}$ é dada por:

$$
\pi\left(\theta_{1}, \theta_{2} \mid D\right)=\frac{L\left(\theta_{1}, \theta_{2}\right) \pi\left(\theta_{1}, \theta_{2}\right)}{\int_{-\infty}^{\infty} \int_{0}^{\infty} L\left(\theta_{1}, \theta_{2}\right) \pi\left(\theta_{1}, \theta_{2}\right) d \theta_{1} d \theta_{2}},
$$

onde $L\left(\theta_{1}, \theta_{2}\right)$ é a função de verossimilhança (4.4).

Pelo teorema de Bayes, se a função de verossimilhança for multiplicada por uma constante arbitrária, as inferências sobre a distribuição da densidade a posteriori não serão afetadas. Assim,

$$
\pi\left(\theta_{1}, \theta_{2} \mid D\right) \propto L\left(\theta_{1}, \theta_{2}\right) \pi\left(\theta_{1}, \theta_{2}\right) .
$$

Substituindo (4.4) e (4.20) temos a densidade a posteriori conjunta para $\theta_{1}$ e $\theta_{2}$, dada por:

$$
\pi\left(\theta_{1}, \theta_{2} \mid D\right) \propto \theta_{1}^{-(n+1)} \exp \left\{-\theta_{2} \sum_{i=1}^{n} x_{i}-\frac{1}{\theta_{1}} \sum_{i=1}^{n} t_{i} e^{-\theta_{2} x_{i}}\right\}
$$


onde $\theta_{1}$ e $\theta_{2} \in \Theta$.

Em análise Bayesiana, inferências são tipicamente baseadas nas densidades a posteriori marginais dos parâmetros envolvidos. Portanto com a posteriori conjunta (4.22) podemos encontrar as densidades a posteriori marginais para $\theta_{1}$ e $\theta_{2}$. Em termos práticos os objetivos dos pesquisadores estão mais voltados para inferências sobre a função de sobrevivência $R$.

\subsubsection{Densidade a Posteriori Marginal para a Função de Sobrevivência num Dado Tempo $t_{0}$}

Nesta subseção, considerando os tempos de sobrevivência originais, é possível encontrar a densidade a posteriori para o parâmetro de interesse $R\left(t_{0}\right)$, a função de sobrevivência num dado tempo $t_{0}$ e num valor especificado $x_{0}$ da covariável.

Considere a transformação de variáveis, dada por:

$$
\left\{\begin{array} { l } 
{ R = \operatorname { e x p } \{ - \frac { t _ { 0 } } { \theta _ { 1 } e ^ { \theta _ { 2 } x _ { 0 } } } \} } \\
{ \phi _ { 2 } = \theta _ { 2 } }
\end{array} \quad \text { então } \quad \left\{\begin{array}{l}
\theta_{1}=\frac{t_{0}}{(-\ln R) e^{\phi_{2} x_{0}}} \\
\theta_{2}=\phi_{2}
\end{array}\right.\right.
$$

onde a matriz Jacobiana é dada por: 


$$
J=\left(\begin{array}{ll}
\frac{\partial \theta_{1}}{\partial R} & \frac{\partial \theta_{1}}{\partial \phi_{2}} \\
\frac{\partial \theta_{2}}{\partial R} & \frac{\partial \theta_{2}}{\partial \phi_{2}}
\end{array}\right)
$$

ou seja,

$$
J=\left(\begin{array}{cc}
\frac{t_{0}}{\operatorname{Re}^{\phi_{2} x_{0}}(-\ln R)^{2}} & -\frac{t_{0} x_{0}}{\ln \operatorname{Re}^{\phi_{2} x_{0}}} \\
0 & 1
\end{array}\right)
$$

Assim, $\operatorname{det}(J)=\frac{t_{0}}{\operatorname{Re}^{\phi_{2} x_{0}}(-\ln R)^{2}}$.

Portanto, a densidade a priori para $R$ e $\phi_{2}$ é dada (de (4.20)), por:

$$
\pi\left(R, \phi_{2}\right) \propto \frac{1}{R(-\ln R)} \quad 0 \leq R \leq 1,-\infty<\phi_{2}<\infty .
$$

Com essa priori, a densidade a posteriori conjunta para $R$ e $\phi_{2}$ é dada por:

$$
\pi\left(R, \phi_{2} \mid D\right) \propto \frac{(-\ln R)^{n-1} e^{n \phi_{2} x_{0}}}{R} \exp \left\{-\phi_{2} \sum_{i=1}^{n} x_{i}+\frac{\ln R}{t_{0}} \sum_{i=1}^{n} t_{i} e^{-\left(x_{i}-x_{0}\right) \phi_{2}}\right\},
$$

onde $0<R<1,-\infty<\phi_{2}<\infty$. 
Integrando a densidade conjunta (4.25) em relação ao parâmetro $\phi_{2}$, obtemos a densidade a posteriori marginal para $R$,

$$
\pi(R \mid D) \propto \frac{(-\ln R)^{n-1}}{R} \int_{-\infty}^{\infty} e^{n \phi_{2} x_{0}} \exp \left\{-\phi_{2} \sum_{i=1}^{n} x_{i}+\frac{\ln R}{t_{0}} \sum_{i=1}^{n} t_{i} e^{-\left(x_{i}-x_{0}\right) \phi_{2}}\right\} d \phi_{2}
$$

Como existe dificuldade para resolvermos analiticamente a integral em (4.26), usamos um método para aproximação de integrais (ver capítulo 2). Assim, usando o método de Laplace, a densidade (4.26) reescrita de forma adequada é dada por:

$$
\pi(R \mid D) \propto \frac{(-\ln R)^{n-1}}{R} \int_{-\infty}^{\infty} \exp \left\{-n h_{R}\left(\phi_{2}\right)\right\} d \phi_{2},
$$

onde $-n h_{R}\left(\phi_{2}\right)=n \phi_{2} x_{0}-\phi_{2} \sum_{i=1}^{n} x_{i}+\frac{\ln R}{t_{0}} \sum_{i=1}^{n} t_{i} e^{-\left(x_{i}-x_{0}\right) \phi_{2}}$.

A densidade a posteriori marginal para $R$ aproximada pelo método de Laplace (ver (2.5)) é dada por:

$$
\pi(R \mid D) \propto \frac{(-\ln R)^{n-3 / 2} e^{n \hat{\phi}_{2} x_{0}}}{R\left\{\sum_{i=1}^{n}\left(x_{0}-x_{i}\right)^{2} t_{i} e^{-\left(x_{i}-x_{0}\right) \hat{\phi}_{2}}\right\}^{-1 / 2}} \exp \left\{-\hat{\phi}_{2} \sum_{i=1}^{n} x_{i}+\frac{\ln R}{t_{0}} \sum_{i=1}^{n} t_{i} e^{-\left(x_{i}-x_{0}\right) \hat{\phi}_{2}}\right\},
$$

onde $0 \leq R \leq 1$ e $\hat{\phi}_{2}$ maximiza $-n h_{R}\left(\phi_{2}\right)$ (ver (4.27)). 
Usando a densidade a posteriori marginal para $R$ em (4.28), é possível fazer inferências sobre o parâmetro. Por exemplo, é possível construir intervalos HPD (ver apêndice A.2) para $R$, ou ainda determinar a moda a posteriori da densidade (4.28).

\subsubsection{Uma Reparametrização para $R(t)$}

No contexto Bayesiano é importante verificarmos se a utilização de diferentes reparametrizações proporciona alguma vantagem computacional e/ou melhoria em termos de aproximações na obtenção da densidade a posteriori para o parâmetro de interesse $R$.

Como podemos observar no capítulo 3, a reparametrização de Guerrero e Johnson (ver Guerrero e Johnson, 1982) considerada para o parâmetro $R$, apresenta a vantagem da transformação ser inversível.

Assim, considerando a reparametrização (3.10), temos a transformação de variáveis,

$$
\left\{\begin{array} { l } 
{ \phi _ { G J } ( \lambda ) = ( \frac { R } { 1 - R } ) ^ { \lambda } - 1 } \\
{ \phi _ { 2 } = \phi _ { 2 } , }
\end{array} \left\{\begin{array}{l}
R=\frac{1}{1+\left(\phi_{G J}+1\right)^{-1 / \lambda}} \\
\phi_{2}=\phi_{2} .
\end{array}\right.\right.
$$

e a matriz Jacobiana dessa transformação é dada por: 
$J\left(R, \phi_{2}\right)=\left|\begin{array}{cc}\frac{1 / \lambda\left(\phi_{G J}+1\right)^{(-1 / \lambda)-1}}{\left(1+\left(\phi_{G J}+1\right)^{-1 / \lambda}\right)^{2}} & 0 \\ 0 & 1\end{array}\right|$.

Portanto, $\operatorname{det}\left(J\left(R, \phi_{2}\right)\right)=\frac{\left(\phi_{G J}+1\right)^{(-1 / \lambda)-1}}{\lambda\left(1+\left(\phi_{G J}+1\right)^{-1 / \lambda}\right)^{2}}$.

A densidade a posteriori conjunta para $\phi_{G J}$ e $\phi_{2}$, determinada a partir da densidade a posteriori conjunta (4.25), é dada por:

$$
\begin{aligned}
& \pi\left(\phi_{G J}, \phi_{2} \mid D\right) \propto \frac{\left[\ln \left(1+\left(\phi_{G j}+1\right)^{-1 / \lambda}\right)\right]^{n-1}}{\left[1+\left(\phi_{G j}+1\right)^{-1 / \lambda}\right]}\left(e^{x_{0} \phi_{2}}\right)^{n}\left(\phi_{G J}+1\right)^{-(1 / \lambda+1)} \times \\
& \times \exp \left\{-\phi_{2} \sum_{i=1}^{n} x_{i}-\frac{\ln \left[1+\left(\phi_{G J}+1\right)^{-1 / \lambda}\right]}{t_{0}} \sum_{i=1}^{n} t_{i} e^{-\left(x_{i}-x_{0}\right) \phi_{2}}\right\}
\end{aligned}
$$

onde $-1<\phi_{G J}<\infty,-\infty<\phi_{2}<\infty$.

A densidade a posteriori marginal para $\phi_{G J}$ é dada por:

$$
\begin{aligned}
\pi\left(\phi_{G J} \mid D\right) \propto & \frac{\left[\ln \left(1+\left(\phi_{G J}+1\right)^{-1 / \lambda}\right)\right]^{n-1}}{\left(1+\left(\phi_{G J}+1\right)^{-1 / \lambda}\right)}\left(\phi_{G J}+1\right)^{-\left(\frac{1}{\lambda}+1\right)} \times \\
& \times \int_{-\infty}^{\infty}\left(e^{x_{0}}\right)^{n \phi_{2}} \exp \left\{-\phi_{2} \sum_{i=1}^{n} x_{i}-\frac{\ln \left[1+\left(\phi_{G J}+1\right)^{-1 / \lambda}\right]}{t_{0}} \sum_{i=1}^{n} t_{i} e^{-\left(x_{i}-x_{0}\right) \phi_{2}}\right\} d \phi_{2} .
\end{aligned}
$$


Para usar o método de Laplace para aproximações de integrais (ver capítulo 2), a densidade (4.32) pode ser reescrita da seguinte forma:

$$
\pi\left(\phi_{G J} \mid D\right) \propto \frac{\left[B\left(\phi_{G J}\right)\right]^{n-1}}{\left[1+A\left(\phi_{G J}\right)\right]}\left(\phi_{G J}+1\right)^{-\left(\frac{1}{\lambda}+1\right)} \int_{-\infty}^{\infty} \exp \left\{-n h_{\phi_{G J}}\left(\phi_{2}\right)\right\} d \phi_{2}
$$

onde,

$$
\left\{\begin{array}{l}
A\left(\phi_{G J}\right)=\left(\phi_{G J}+1\right)^{-1 / 2} \\
B\left(\phi_{G J}\right)=\ln \left[1+A\left(\phi_{G J}\right)\right] \\
A^{*}\left(\phi_{2}\right)=\sum_{i=1}^{n} t_{i} e^{-\left(x_{i}-x_{0}\right) \phi_{2}} \\
\mathrm{e}-n h_{\phi_{G}}\left(\phi_{2}\right)=n \phi_{2} x_{0}-\phi_{2} \sum_{i=1}^{n} x_{i}-\frac{B\left(\phi_{G J}\right) A^{*}\left(\phi_{2}\right)}{t_{0}}
\end{array}\right.
$$

A densidade a posteriori marginal para $\phi_{G J}$ obtida pelo método de Laplace (ver capítulo 2) é dada por:

$$
\pi\left(\phi_{G J} \mid D\right) \propto \frac{\left[B\left(\phi_{G J}\right)\right]^{n-3 / 2}\left(e^{x_{0} \phi_{2}}\right)^{n}\left(\phi_{G J}+1\right)^{-\left(\frac{1}{2}+1\right)}}{\left[1+A\left(\phi_{G J}\right)\right]\left[A^{* \prime}\left(\hat{\phi}_{2}\right)\right]^{1 / 2}} \exp \left\{-\hat{\phi}_{2} \sum_{i=1}^{n} x_{i}-\frac{B\left(\phi_{G J}\right) A^{*}\left(\hat{\phi}_{2}\right)}{t_{0}}\right\}
$$

onde, $-1<\phi_{G J}<\infty$ e $\hat{\phi}_{2}$ maximiza $-n h_{G J}\left(\phi_{2}\right)$ dado em (4.33). 
Para escolhermos o valor apropriado de $\lambda$ na parametrização de GuerreroJohnson $\phi_{G J}(\lambda)$, podemos usar a terceira derivada padronizada da verossimilhança "profile" ou da densidade a posteriori marginal para $\phi_{G J}(\lambda)$ (ver (3.7)) tal que este valor seja próximo de zero. Também podemos utilizar o "t-plot" de Hills e Smith (1993) (ver (3.12)) para verificar graficamente a adequabilidade da reparametrização considerada.

Usando a densidade a posteriori marginal (4.34) é possível obtermos melhores inferências sobre a função de sobrevivência $R$. 


\section{CAPÍTULO 5}

\section{MODELO DE REGRESSÃO LOCAÇÃO- ESCALA COM DISTRIBUIÇÃO DE VALOR EXTREMO}

A distribuição de valor extremo é um dos modelos mais utilizados em confiabilidade, dada a sua relação com a distribuição Weibull. Para inferências sobre os parâmetros deste modelo ou funções desses parâmetros, usualmente são considerados resultados assintóticos. Um desses resultados é dado pela normalidade assintótica dos estimadores de máxima verossimilhança.

Um problema prático e de grande interesse para os estatísticos está relacionado aos resultados assintóticos precisos, considerando tamanhos de amostras pequenas ou moderadas.

Neste capítulo novamente exploramos uma reparametrização que melhora a aproximação normal da função de verossimilhança especialmente para tamanhos de amostras pequenas ou moderadas, melhorando assim os resultados assintóticos. Também apresentaremos uma análise Bayesiana do modelo de regressão locaçãoescala com a distribuição de valor extremo introduzida no capítulo 1. 


\subsection{Aproximação Normal para os Estimadores de Máxima Verossimilhança}

Um método comum para estimar parâmetros de uma distribuição de sobrevivência é o método de máxima verossimilhança, onde os estimadores são valores que maximizam a função de verossimilhança e têm boas propriedades assintóticas quando os tamanhos amostrais são suficientemente grandes. Nesta seç̧ão trataremos da utilização da normalidade assintótica dos estimadores de máxima verossimilhança para os parâmetros da distribuição de valor extremo e o caso especial do estimador de máxima verossimilhança para a função de sobrevivência.

Consideremos o modelo de locação-escala com a distribuição de valor extremo dado em (1.23) capítulo 1 ,

$$
f(y \mid \mathbf{x})=\frac{1}{\sigma} \exp \left[\frac{y-\mu(\mathbf{x})}{\sigma}-\exp \left(\frac{y-\mu(\mathbf{x})}{\sigma}\right)\right]-\infty<y<\infty .
$$

Considerando o modelo de regressão $\mu(x)=\mathbf{x} \beta$, o logaritmo da função de verossimilhança para $\beta$ e $\sigma$ com dados censurados é dado por:

$$
\ell(\beta, \sigma)=-r \ln \sigma+\sum_{i \in D} \frac{\left(y_{i}-\mathbf{x}_{i} \beta\right)}{\sigma}-\sum_{i=1}^{n} \exp \left\{\frac{y_{i}-\mathbf{x}_{i} \beta}{\sigma}\right\}
$$

onde ré o número de observações não-censuradas (ver Lawless, 1982).

Para o caso especial do modelo de regressão linear simples dado por: 


$$
x_{i} \beta=\beta_{0}+\beta_{1} x_{i}
$$

o logaritmo da função de verossimilhança para $\beta_{0}, \beta_{1}$ e $\sigma$, considerando dados com censuras, é dado por:

$$
\ell\left(\beta_{0}, \beta_{1}, \sigma\right)=-n \log \sigma+\sum_{i \in D}\left(\frac{y_{i}-\beta_{0}-\beta_{1} x_{i}}{\sigma}\right)-\sum_{i=1}^{n} \exp \left(\frac{y_{i}-\beta_{0}-\beta_{1} x_{1}}{\sigma}\right)
$$

e a matriz de informação observada $I_{0}$ é dada por:

$$
I_{0}=\frac{1}{\hat{\sigma}^{2}}\left(\begin{array}{ccc}
r & \sum_{i \in D} x_{i} & r+\sum_{i \in D} \hat{z}_{i} \\
\sum_{i \in D} x_{i} & \sum_{i=1}^{n} x_{i}^{2} e^{\hat{z}_{i}} & \sum_{i=1}^{n} x_{i} \hat{z}_{i} e^{\hat{z}_{i}} \\
r+\sum_{i \in D} \hat{z}_{i} & \sum_{i=1}^{n} x_{i} \hat{z}_{i} e^{\hat{z}_{i}} & r+\sum_{i=1}^{n} \hat{z}_{i}^{2} e^{\hat{z}_{i}}
\end{array}\right),
$$

onde $\hat{z}_{i}=\left(y_{i}-x_{i} \hat{\beta}\right) / \hat{\sigma}$.

Supondo dados sem censuras e a variável aletória $z$ com a densidade de valor extremo padronizada $\exp \left(z-e^{z}\right),-\infty<z<\infty$, temos $E\left(z_{i}\right)=-\gamma, E\left(e^{z_{i}}\right)=1$, $E\left(z_{i} e^{z_{i}}\right)=1-\gamma, E\left(z_{i}^{2} e^{z_{i}}\right)=\frac{\pi^{2}}{6}+\gamma^{2}-2 \gamma$, onde $(\gamma=0.5772 \cdots$ constante de Euler $)$ (ver Lawless, 1982).

Daí, a matriz de informação de Fisher para $\beta$ e $\sigma$ do modelo (5.3) é dada por: 


$$
I=\frac{1}{\sigma^{2}}\left(\begin{array}{ccc}
n & \sum_{i=1}^{n} x_{i} & n(1-\gamma) \\
\sum_{i=1}^{n} x_{i} & \sum_{i=1}^{n} x_{i}^{2} & (1-\gamma) \sum_{i=1}^{n} x_{i} \\
n(1-\gamma) & (1-\gamma) \sum_{i=1}^{n} x_{i} & n\left[1+\left(\frac{\pi^{2}}{6}+\gamma^{2}-2 \gamma\right)\right]
\end{array}\right)
$$

Para valores grandes de $n$, os estimadores de máxima verossimilhança $\hat{\beta}_{0}, \hat{\beta}_{1}$ e $\hat{\sigma}$ têm uma distribuição normal assintótica $N\left\{\left(\beta_{0}, \beta_{1}, \sigma\right) ; I^{-1}\left(\hat{\beta}_{0}, \hat{\beta}_{1}, \hat{\sigma}\right)\right\}$. Em trabalhos práticos é usual considerar a matriz de informação observada $I_{0}$ no lugar da matriz de informação de Fisher $I$.

Intervalos de confiança e testes de hipóteses para os parâmetros $\beta_{0}, \beta_{1}$ e $\sigma$ também podem ser determinados pela distribuição normal assintótica dos estimadores de máxima verossimilhança.

\subsection{Estimação da Função de Sobrevivência}

Em análises de dados médicos do tempo de vida ou no estudo de confiabilidade na engenharia, em geral o pesquisador tem interesse em obter inferências sobre a função de sobrevivência de pacientes ou componentes respectivamente, em um tempo especificado $t_{0}$. Nesta secção, desenvolveremos técnicas de estimação para a função de sobrevivência $R\left(y^{*}\right)$, considerando a distribuição de valor extremo para o logaritmo dos tempos de vida $\left(y^{*}=\ln \left(t_{0}\right)\right)$. Assumindo o modelo $\log$-linear (5.3), a função de sobrevivência em um tempo fixado $y^{*}=\ln \left(t_{0}\right) \operatorname{com} x=x^{*}$ é dada por: 


$$
R\left(y^{*}\right)=P\left(y>y^{*}\right)=\exp \left\{-\exp \left(\frac{y^{*}-\beta_{0}-\beta_{1} x^{*}}{\sigma}\right)\right\}
$$

onde, $\ln \left(-\ln R\left(y^{*}\right)\right)=\frac{1}{\sigma}\left(y^{*}-\beta_{0}-\beta_{1} x^{*}\right)$.

Considere a transformação $\left(\beta_{0}, \beta_{1}, \sigma\right)$ em $\left(\beta_{0}, R, \sigma\right)$, onde $R=R\left(y^{*}\right)$ num dado valor $x^{*}$ da covariável, dada por:

$$
\left\{\begin{array}{l}
\ln \left(-\ln (R)=\frac{1}{\sigma}\left(y^{*}-\beta_{0}-\beta_{1} x^{*}\right)\right. \\
\beta_{0}=\beta_{0} \\
\sigma=\sigma
\end{array}\right.
$$

O logaritmo da função de verossimilhança em termos de $R=R\left(y^{*}\right)$ é dado por:

$\ell\left(R, \beta_{0}, \sigma\right)=-n \ln \sigma+\frac{n \bar{y}}{\sigma}-\frac{m y^{*} \bar{x}}{\sigma x^{*}}-\frac{n \beta_{0}}{\sigma}+\frac{n \beta_{0} \bar{x}}{\sigma x^{*}}+\frac{n \bar{x}}{x^{*}} \ln (-\ln R)-e^{-\beta_{0} / \sigma} \sum_{i=1}^{n} e^{A_{i}(R)}$

onde, $A_{i}(R)=\frac{1}{\sigma}\left(y_{i}-\frac{y^{*} x_{i}}{x^{*}}\right)+\frac{\beta_{0} x_{i}}{\sigma x^{*}}+\frac{x_{i}}{x^{*}} \ln (-\ln R)$.

Assumindo $\sigma$ e $\beta_{0}$ conhecidos, o logaritmo da função de verossimilhança para $R$ é dado por: 


$$
\ell(R) \propto \frac{n \bar{x}}{x^{*}} \ln (-\ln R)-e^{-\beta_{0} / \sigma} \sum_{i=1}^{n} e^{A_{i}(R)},
$$

onde $A_{i}(R)$ é dado em (5.9).

Nesse caso, o estimador de máxima verossimilhança para a função de sobrevivência $R$ satisfaz a equação:

$$
\hat{R}(-\ln \hat{R}) \sum_{i=1}^{n} A_{i}^{\prime}(\hat{R}) e^{A_{l}(R)}=-\frac{n \bar{x} e^{\beta_{0} / \sigma}}{x^{*}}
$$

onde, $A_{i}^{\prime}(R)=-x_{i} / x^{*} R(-\ln R)$.

Geralmente, inferências em $R$ são baseadas na "normalidade" assintótica dos estimadores de máxima verossimilhança de $R$. A precisão desses resultados assintóticos pode ser muito pobre, especialmente para tamanhos de amostras pequenas ou moderadas. Para verificar a precisão dos resultados assintóticos, podemos considerar uma medida de não-normalidade para a função de verossimilhança baseada na derivada terceira padronizada do logaritmo da função de verossimilhança (ver Capítulo 3) dada por:

$$
S T D(\hat{R})=\left|\frac{\sum_{i=1}^{n} x_{i}^{3} e^{A_{i}(\hat{R})}-3 x^{*}(1+\ln \hat{R}) \sum_{i=1}^{n} x_{i}^{2} e^{A_{i}(\hat{R})}}{\left(e^{-\beta_{0} / \sigma}\right)^{1 / 2}\left[\sum_{i=1}^{n} x_{i}^{2} e^{A_{i}(\hat{R})}\right]^{3 / 2}}\right| .
$$

A "normalidade" da função de verossimilhança é apropriada se $S T D(\hat{R}) \cong 0$. Se, baseado no valor obtido para $\operatorname{STD}(\hat{R})$, concluirmos que a "normalidade" da função de verossimilhança não é apropriada como alternativa podemos explorar diferen- 
tes parametrizações na transformação de $R$, para melhorar a "normalidade" da função de verosssimilhança. Quando $\sigma$ e $\beta_{0}$ são desconhecidos, podemos considerar a função de verossimilhança "profile" $L_{\hat{\beta}_{0}, \hat{\sigma}}(R)$, onde $\hat{\beta}_{0}$ e $\hat{\sigma}$ maximizam a função de verossimilhança para cada valor de $R$.

\subsection{Comparação de Dois Tratamentos}

Em muitas situações de ensaios clínicos, pacientes com doenças graves são colocados em dois ou mais tratamentos, para determinar qual ou se algum dos tratamentos prolonga significativamente o tempo de vida ou de remissão do paciente. Em geral o pesquisador quer verificar se um tratamento novo é melhor que um tratamento tradicional. A literatura apresenta várias técnicas não-paramétricas e paramétricas, com dados censurados ou não, para este problema. Uma grande dificuldade em utilizar modelos paramétricos consiste na identificação de um modelo adequado para os dois grupos. A distribuição de valor extremo apresenta muitos resultados inclusive com dados censurados. Nesta secção apresentamos técnicas de regressão na comparação dos dois tratamentos usando procedimentos de inferência clássica assim como de inferência Bayesiana.

Suponha $n=n_{1}+n_{2}$ pacientes alocados aleatoriamente em dois tratamentos diferentes. Desses pacientes, $n_{1}$ recebem um tratamento padrão (controle) e $n_{2}$ pacientes recebem um tratamento experimental (novo tratamento).

Considere duas amostras com dados sem censuras para os dois grupos de tratamentos: 
Amostra 1: $\quad y_{11}, y_{12}, \ldots, y_{1 m} \quad$ para o tratamento 1 (controle), com $x_{1 i}=0$

Amostra 2: $\quad y_{21}, y_{22}, \ldots, y_{2 m_{2}}$ para o tratamento 2 (novo tratamento), com $x_{2 i}=1$.

Assumindo o modelo log-linear (5.3), o logaritmo da função de verossimilhança para $\beta_{0}, \beta_{1}$ e $\sigma$ para os dois grupos de tratamentos é dado por:

$$
\begin{aligned}
\ell\left(\beta_{0}, \beta_{1}, \sigma\right)= & -n \ln \sigma+\sum_{i=1}^{m_{1}}\left(\frac{y_{1 i}-\beta_{0}}{\sigma}\right)+\sum_{i=1}^{m_{2}}\left(\frac{y_{2 i}-\beta_{0}-\beta_{1}}{\sigma}\right)- \\
& -\sum_{i=1}^{m_{1}} \exp \left(\frac{y_{1 i}-\beta_{0}}{\sigma}\right)-\sum_{i=1}^{m_{2}} \exp \left(\frac{y_{2 i}-\beta_{0}-\beta_{1}}{\sigma}\right)
\end{aligned}
$$

onde $n=n_{1}+n_{2}$ é o tamanho amostral nos dois grupos.

A partir de (5.7), a função de sobrevivência para um paciente num tempo especificado $y^{*}=\ln \left(t_{0}\right)$ no tratamento $2\left(x^{*}=1\right)$ é dado por:

$$
R\left(y^{*}\right)=\exp \left\{-\exp \left(\frac{y^{*}-\beta_{0}-\beta_{1}}{\sigma}\right)\right\}
$$

Assumindo $\beta_{0}$ e $\sigma$ conhecidos, o logaritmo da função de verossimilhança para $R$ (ver (5.10)) é dado por:

$$
\ell(R) \propto n_{2} \ln (-\ln R)+\ln R \sum_{i=1}^{m_{2}} \exp \left(\frac{y_{2 i}-y^{*}}{\sigma}\right) .
$$


Neste caso, o estimador de máxima verossimilhança para a função de sobrevivência $R$ é dado por:

$$
\hat{R}=\exp \left\{-\frac{n_{2}}{\sum_{i=1}^{m_{2}} \exp \left(\frac{y_{2 i}-y^{*}}{\sigma}\right)}\right\} .
$$

Assim, a derivada terceira padronizada do logaritmo da função de verossimilhança localmente em $\hat{R}$ (ver 5.12) é reduzido para:

$$
S T D(\hat{R})=\left|-n_{2}{ }^{-1 / 2}(2+3 \ln \hat{R})\right| \text {. }
$$

A partir de (5.17) observamos que os valores da derivada terceira padronizada localmente no estimador de máxima verossimilhança $\hat{R}$ podem ser grandes se $R \approx 0$ ou $R \approx 1$, especialmente com $n_{2}$ pequeno.

Considerando $\sigma$ e $\beta_{0}$ desconhecidos podemos calcular a verossimilhança "profile" $\ell_{\hat{\beta}_{0}, \hat{\sigma}}(R)$ para cada valor de $R$ da seguinte forma:

$$
\begin{aligned}
\ell_{\hat{\beta}_{0} \hat{\sigma}}(R)=- & n \ln \hat{\sigma}+\sum_{i=1}^{m_{1}}\left(\frac{y_{1 i}-\hat{\beta}_{0}}{\hat{\sigma}}\right)+\sum_{i=1}^{m_{2}}\left(\frac{y_{2 i}-y^{*}}{\hat{\sigma}}\right)+n_{2} \ln (-\ln R)- \\
& -\sum_{i=1}^{m_{1}} \exp \left(\frac{y_{1 i}-\hat{\beta}_{0}}{\hat{\sigma}}\right)-(-\ln R) \sum_{i=1}^{m_{2}} \exp \left(\frac{y_{2 i}-y^{*}}{\hat{\sigma}}\right) .
\end{aligned}
$$


Podemos encontrar o valor de $R$ que maximiza $\ell_{\hat{\beta}_{0}, \hat{\sigma}}(R)$. Este valor pode ser encontrado através de um gráfico de $\ell_{\hat{\boldsymbol{\beta}}_{0}, \hat{\sigma}}(R)$ versus $R$ e assim, calcular $\operatorname{STD}(\hat{R})$. Teremos resultados análogos aos obtidos assumindo $\sigma$ e $\beta_{0}$ conhecidos.

\subsubsection{Reparametrização de Guerrero e Johnson para a Função de Sobrevivência}

Quando temos valores grandes para $S T D(\hat{R})$, podemos explorar diferentes parametrizações para melhorar a "normalidade" da função de verossimilhança. Assim como foi mencionado no capítulo 4, exploramos nesta secção uma parametrização especial que foi introduzida por Guerrero e Johnson (1982) (ver Capítulo 3).

Considerando a reparametrização de Guerrero e Jonhson dada em (3.10) temos a seguinte transformação:

$$
\left\{\begin{array} { l } 
{ \phi _ { G J } = ( \frac { R } { 1 - R } ) ^ { \lambda } - 1 } \\
{ \beta _ { 0 } = \beta _ { 0 } } \\
{ \sigma = \sigma }
\end{array} \Rightarrow \left\{\begin{array}{l}
R=\left[1+\left(\phi_{G J}+1\right)^{-1 / \lambda}\right]^{-1} \\
\beta_{0}=\beta_{0} \\
\sigma=\sigma
\end{array}\right.\right.
$$

Seja $\quad \ln R=-\ln \left[1+A\left(\phi_{G J}\right)\right], \quad$ onde $\quad A\left(\phi_{G J}\right)=\left(\phi_{G J}+1\right)^{-1 / \lambda} \quad \mathrm{e}$ $B\left(\phi_{G J}\right)=\ln \left[1+A\left(\phi_{G J}\right)\right]$. O logaritmo da função de verossimilhança para $\phi_{G J}, \beta_{0} \mathrm{e}$ $\sigma \operatorname{com} x=x^{*}$ é dado por: 
$\ell\left(\phi_{G J}, \beta_{0}, \sigma\right)=-n \ln \sigma+\frac{n \bar{y}}{\sigma}-\frac{n y^{*} \bar{x}}{\sigma x^{*}}-\frac{n \beta_{0}}{\sigma}+\frac{n \beta_{0} \bar{x}}{\sigma x^{*}}+\frac{n \bar{x}}{x} \ln B\left(\phi_{G j}\right)-e^{-\beta_{0} / \sigma} \sum_{i=1}^{n} e^{A_{i}\left(\phi_{G /} \beta_{0}, \sigma\right)}$,

onde $A_{i}\left(\phi_{G J}, \beta_{0}, \sigma\right)=\frac{1}{\sigma}\left(y_{i}-\frac{y^{*} x_{i}}{x^{*}}\right)+\frac{\beta_{0} x_{i}}{\sigma x^{*}}+\frac{x_{i}}{x^{*}} \ln B\left(\phi_{G J}\right)$.

Assumindo $\beta_{0}$ e $\sigma$ conhecidos, o logaritmo da função de verossimilhança para $\phi_{G J}$, a partir de (5.20), é dado por:

$$
\ell\left(\phi_{G J}\right) \propto \frac{n \bar{x}}{x^{*}} \ln B\left(\phi_{G J}\right)-e^{-\beta_{0} / \sigma} \sum_{i=1}^{m_{2}} e^{A_{i}\left(\phi_{G J}\right)}
$$

onde $A_{i}\left(\phi_{G J}\right)=\frac{1}{\sigma}\left(y_{i}-\frac{y^{*} x_{i}}{x^{*}}\right)+\frac{\beta_{0} x_{i}}{\sigma x^{*}}+\frac{x_{i}}{x^{*}} \ln B\left(\phi_{G J}\right)$ e $B\left(\phi_{G J}\right)$ é definido em (5.19).

Neste caso o estimador de máxima verossimilhança para $\phi_{G J}$, obtido de $\ell^{\prime}\left(\phi_{G J}\right)=0$, satisfaz a equação:

$$
\sum_{i=1}^{n} x_{i} e^{A_{i}\left(\phi_{G J}\right)}=n \bar{x} e^{\beta_{0} / \sigma}
$$

onde podemos solucionar (5.22) usando um método iterativo como por exemplo o método iterativo de Newton-Raphson.

Considerando o caso especial de comparação de dois tratamento onde $x^{*}=1$ (novo tratamento), o logaritmo da função de verossimilhança (5.21) é dado por:

$$
\ell\left(\phi_{G J}\right) \propto n_{2} \ln B\left(\phi_{G J}\right)-B\left(\phi_{G J}\right) \sum_{i=1}^{m} \exp \left(\frac{y_{2 i}-y^{*}}{\sigma}\right)
$$


Neste caso o estimador de máxima verossimilhança para $\phi_{G J}$ satisfaz a equação,

$$
B\left(\hat{\phi}_{G J}\right)=\frac{m_{2}}{\sum_{i=1}^{m_{2}} \exp \left(\frac{y_{2 i}-y^{*}}{\sigma}\right)} .
$$

Para determinar qual é o valor mais apropriado de $\lambda$ que forneça boa "normalidade" para o logaritmo da função de verossimilhança $\ell\left(\phi_{G J}\right)$ dado em (5.23), utilizamos a derivada terceira padronizada do logaritmo da função de verossimilhança localmente no estimador de máxima verossimilhança $\hat{\phi}_{G J}(\lambda)$ dado por:

$$
\operatorname{STD}\left(\hat{\phi}_{G J}\right)=\mid n_{2}^{-1 / 2} \frac{B\left(\hat{\phi}_{G J}\right)}{B^{\prime}\left(\hat{\phi}_{G J}\right)}\left[2-\frac{3 B^{\prime \prime}\left(\hat{\phi}_{G J}\right)}{B^{\prime}\left(\hat{\phi}_{G J}\right)}\right] .
$$

Assim, o valor de $\lambda$ apropriado que melhora a "normalidade" da função de verossimilhança nas duas situações amostrais (ver secção 5.3) tal que - $\operatorname{STD}\left(\hat{\phi}_{G J}\right) \cong 0$, é obtido através de um método gráfico ou um método iterativo.

Com $\lambda$ obtido tal que $\operatorname{STD}\left(\hat{\phi}_{G J}\right) \cong 0$, podemos considerar a "normalidade" assintótica de $\hat{\phi}_{G J}(\lambda)$ e conseguir melhores inferências especialmente para amostras pequenas ou moderadas.

Quando $\beta_{0}$ e $\sigma$ são desconhecidos, podemos considerar o logaritmo da função de verossimilhança "profile" $\ell_{\hat{\beta}_{0}, \hat{\sigma}}\left(\phi_{G J}(\lambda)\right)$ para cada valor de $\ell_{\hat{\beta}_{0}, \hat{\sigma}}\left(\phi_{G J}(\lambda)\right)$ dada por: 


$$
\begin{aligned}
\ell_{\hat{\beta}_{0}, \hat{\sigma}}\left(\phi_{G J}\right)= & -n \ln \hat{\sigma}+\sum_{i=1}^{m_{1}}\left(\frac{y_{1 i}-\hat{\beta}_{0}}{\hat{\sigma}}\right)+\sum_{i=1}^{m_{2}}\left(\frac{y_{2 i}-y^{*}}{\hat{\sigma}}\right)+n_{2} \ln B\left(\phi_{G J}\right) \\
& -\sum_{i=1}^{m_{1}} \exp \left(\frac{y_{1 i}-\hat{\beta}_{0}}{\hat{\sigma}}\right)-B\left(\phi_{G J}\right) \sum_{i=1}^{m_{2}} \exp \left(\frac{y_{2 i}-y^{*}}{\hat{\sigma}}\right)
\end{aligned}
$$

O valor de $\phi_{G J}(\lambda)$ que maximiza $\ell_{\hat{\beta}_{0}, \hat{\sigma}}\left(\phi_{G J}(\lambda)\right)$ pode ser encontrado através de um gráfico de $\ell_{\hat{\beta}_{0}, \hat{\sigma}}\left(\phi_{G J}(\lambda)\right)$ versus $\phi_{G J}(\lambda)$.

\subsubsection{Análise Bayesiana Assumindo $\beta_{0}, \beta_{1}$ e $\sigma$ Desconhecidos}

Assim como foi mencionado no capítulo 4 secção (4.2), nesta subsecção será desenvolvida uma análise Bayesiana para o modelo de locação-escala com distribuição de valor extremo considerando o caso especial de dois tratamentos. Considerando uma priori não-informativa, será obtida a densidade a posteriori para parâmetros de interesse.

\subsubsection{Densidades a Priori e a Posteriori}

Vamos assumir que o pesquisador não tem conhecimento a priori a respeito da distribuição dos parâmetros $\beta_{0}, \beta_{1}$ e $\sigma$. Quando não temos nenhuma informação a respeito dos parâmetros, devemos formular uma priori tal que toda informação 
sobre os parâmetros venha através dos dados, ou seja, devemos considerar uma priori não-informativa, em seguida combinando a informação a priori com a informação contida nos dados obtemos a densidade a posteriori para o parâmetro de interesse.

Usando a regra de Jeffreys (ver por exemplo, Box e Tiao, 1973), a densidade a priori para $\beta_{0}, \beta_{1}$ e $\sigma$ é dada por:

$$
\pi\left(\beta_{0}, \beta_{1}, \sigma\right) \propto\left\{\operatorname{det} I\left(\beta_{0}, \beta_{1}, \sigma\right)\right\}^{1 / 2},
$$

onde $I\left(\beta_{0}, \beta_{1}, \sigma\right)$ é a matriz de informação de Fisher dada por:

$$
I\left(\beta_{0}, \beta_{1}, \sigma\right)=\left(\begin{array}{ccc}
E\left\{-\frac{\partial^{2} \ell}{\partial \beta_{0}^{2}}\right\} & E\left\{-\frac{\partial^{2} \ell}{\partial \beta_{0} \partial \beta_{1}}\right\} & E\left\{-\frac{\partial^{2} \ell}{\partial \beta_{0} \partial \sigma}\right\} \\
E\left\{-\frac{\partial^{2} \ell}{\partial \beta_{1} \partial \beta_{0}}\right\} & E\left\{-\frac{\partial^{2} \ell}{\partial \beta_{1}^{2}}\right\} & E\left\{-\frac{\partial^{2} \ell}{\partial \beta_{1} \partial \sigma}\right\} \\
E\left\{-\frac{\partial^{2} \ell}{\partial \sigma \partial \beta_{0}}\right\} & E\left\{-\frac{\partial^{2} \ell}{\partial \sigma \partial \beta_{1}}\right\} & E\left\{-\frac{\partial^{2} \ell}{\partial \sigma^{2}}\right\}
\end{array}\right),
$$

e $\ell$ é o logaritmo da função de verossimilhança definido em (5.13).

Considerando o problema de duas amostras, a matriz de informação de Fisher é dada por: 


$$
I\left(\beta_{0}, \beta_{1}, \sigma\right)=\frac{1}{\sigma^{2}}\left[\begin{array}{ccc}
n & n_{2} & n(1-\gamma) \\
n_{2} & n_{2} & n_{2}(1-\gamma) \\
n(1-\gamma) & n_{2}(1-\gamma) & n\left(\frac{\pi^{2}}{6}+\gamma^{2}-3 \gamma+1\right)
\end{array}\right]
$$

No cálculo do determinante de $I\left(\beta_{0}, \beta_{1}, \sigma\right)$, isto é, det $I\left(\beta_{0}, \beta_{1}, \sigma\right)$, note que a expressão $n\left(n_{2}^{2}-n_{2} n\right)\left[(1-\gamma)^{2}-\left(\frac{\pi^{2}}{6}+\gamma^{2}-3 \gamma+1\right)\right]$ é constante em relação aos parâmetros. Assim,

$$
\operatorname{det} I\left(\beta_{0}, \beta_{1}, \sigma\right) \propto \frac{1}{\sigma^{2}}
$$

portanto, uma priori não informativa de Jeffreys para $\beta_{0}, \beta_{1}$ e $\sigma$ de (5.27) é dada por:

$$
\pi\left(\beta_{0}, \beta_{1}, \sigma\right) \propto \frac{1}{\sigma} \quad \sigma>0
$$

Podemos combinar a informação a priori sobre os parâmetros com as informações obtida nos dados, obtendo assim uma distribuição a posteriori da seguinte forma:

$$
\pi\left(\beta_{0}, \beta_{1}, \sigma \mid D\right) \propto L\left(\beta_{0}, \beta_{1}, \sigma\right) \pi\left(\beta_{0}, \beta_{1}, \sigma\right)
$$


Assim, considerando a densidade a priori (5.28) e a função de verossimilhança (5.13), a densidade a posteriori conjunta para esses parâmetros é dada por:

$$
\begin{aligned}
\pi\left(\beta_{0}, \beta_{1}, \sigma \mid D\right) & \propto \sigma^{-(n+1)} \exp \left\{\sum_{i=1}^{m_{1}}\left(\frac{y_{1 i}-\beta_{0}}{\sigma}\right)+\sum_{i=1}^{m_{2}}\left(\frac{y_{2 i}-\beta_{0}-\beta_{1}}{\sigma}\right)-\right. \\
& \left.-\sum_{i=1}^{m_{1}} \exp \left(\frac{y_{1 i}-\beta_{0}}{\sigma}\right)+\sum_{i=1}^{m_{2}} \exp \left(\frac{y_{2 i}-\beta_{0}-\beta_{1}}{\sigma}\right)\right\},
\end{aligned}
$$

onde $\beta_{0}, \beta_{1} \in \Theta$ e $\sigma>0$.

\subsubsection{Posteriori Marginal para $\boldsymbol{R}$}

A partir da densidade a posteriori conjunta para os parâmetros $\beta_{0}, \beta_{1}$ e $\sigma$, $\pi\left(\beta_{0}, \beta_{1}, \sigma \mid D\right)$ (ver (5.29)) e considerando que os dados tem distribuição de valor extremo, podemos encontrar a densidade a posteriori marginal para $R$, onde $R$ é a função de sobrevivência dos dados com distribuição de valor extremo dado em (5.14), assumindo duas amostras com $x^{*}=1$.

Considerando a transformação dada em (5.8) e usando a técnica de transformação de variáveis, temos:

$$
\left\{\begin{array}{l}
\beta_{1}=y^{*}-\beta_{0}-\sigma \ln (-\ln R) \\
\beta_{0}=\beta_{0} \\
\sigma=\sigma
\end{array}\right.
$$


e a matriz Jacobiana é dada por:

$$
J=\left(\begin{array}{lll}
\partial \beta_{1} / \partial R & \partial \beta_{1} / \partial \beta_{0} & \partial \beta_{1} / \partial \sigma \\
\partial \beta_{0} / \partial R & \partial \beta_{0} / \partial \beta_{0} & \partial \beta_{0} / \partial \sigma \\
\partial \sigma / \partial R & \partial \sigma / \partial \beta_{0} & \partial \sigma / \partial \sigma
\end{array}\right)
$$

ou seja,

$$
J=\left(\begin{array}{ccc}
\frac{\sigma}{R(-\ln R)} & -1 & \ln (-\ln R) \\
0 & 1 & 0 \\
0 & 0 & 1
\end{array}\right)
$$

portanto, $\operatorname{det}(J)=\frac{\sigma}{R \ln (-\ln R)}$

A partir da transformação de variáveis, a densidade a posteriori conjunta para $R, \beta_{0}$ e $\sigma$ é dada por:

$$
\begin{aligned}
\pi\left(R, \beta_{0}, \sigma\right) \propto & \frac{\sigma^{-n}}{R \ln (-\ln R)} \exp \left\{\sum_{i=1}^{m_{1}}\left(\frac{y_{1 i}-\beta_{0}}{\sigma}\right)+\sum_{i=1}^{m_{2}}\left(\frac{y_{2 i}-y^{\bullet}}{\sigma}\right)-\right. \\
& \left.-\sum_{i=1}^{m_{1}} \exp \left(\frac{y_{1}-\beta_{0}}{\sigma}\right)+\ln R \sum_{i=1}^{m_{2}} \exp \left(\frac{y_{2 i}-y^{\bullet}}{\sigma}\right)\right\}
\end{aligned}
$$

onde $0 \leq R \leq,-\infty<\beta_{0}<\infty$ e $\sigma>0$. 
A densidade a posteriori marginal para $R$ é dada por:

$$
\begin{gathered}
\pi(R \mid D) \propto \frac{(-\ln R)^{m_{2}-1}}{R} \iint \sigma^{-n} \exp \left\{\sum_{i=1}^{m_{1}}\left(\frac{y_{1 i}-\beta_{0}}{\sigma}\right)+\sum_{i=1}^{m_{2}}\left(\frac{y_{2 i}-y^{*}}{\sigma}\right)-\right. \\
\left.-\sum_{i=1}^{m_{1}} \exp \left(\frac{y_{1 i}-\beta_{0}}{\sigma}\right)-\sum_{i=1}^{m_{2}} \exp \left(\frac{y_{2 i}-y^{*}}{\sigma}\right)\right\} d \beta_{0} d \sigma .
\end{gathered}
$$

Para usar o método de Laplace para aproximações de integrais (ver capítulo 2), a densidade $\pi(R \mid D)$ deve ser rescrita da seguinte forma:

$$
\pi(R \mid D) \propto \frac{1}{R(-\ln R)^{1-m_{2}}} \iint \exp \left\{-n h_{R}\left(\beta_{0}, \sigma\right)\right\} d \beta_{0} d \sigma
$$

onde,

$-n h_{R}\left(\beta_{0}, \sigma\right)=-n \ln \sigma+\sum_{i=1}^{n_{1}}\left(\frac{y_{1 i}-\beta_{0}}{\sigma}\right)+\sum_{i=1}^{m_{2}}\left(\frac{y_{2 i}-y^{*}}{\sigma}\right)-\sum_{i=1}^{m_{1}} \exp \left(\frac{y_{1 i}-\beta_{0}}{\sigma}\right)-\sum_{i=1}^{m_{2}} \exp \left(\frac{y_{2 i}-y^{*}}{\sigma}\right)$

Assim pelo método de Laplace, a densidade a posteriori marginal para $R$ é dada por:

$$
\begin{aligned}
\pi(R \mid D) \propto & \frac{(-\ln \hat{R})^{m_{2-1}}}{\hat{R} \Delta^{m_{1}}} \hat{\sigma}^{-n} \times \exp \left\{\sum_{i=1}^{m_{1}}\left(\frac{y_{1 i}-\hat{\beta}_{0}}{\sigma}\right)+\right. \\
& \left.+\sum_{i=1}^{m_{2}}\left(\frac{y_{2 i}-y^{*}}{\hat{\sigma}}\right)-\sum_{i=1}^{m_{1}} \exp \left(\frac{y_{1 i}-\hat{\beta}_{0}}{\hat{\sigma}}\right)-(-\ln R) \sum_{i=1}^{m_{2}} \exp \left(\frac{y_{2 i}-y^{*}}{\hat{\sigma}}\right)\right\}(5
\end{aligned}
$$


onde, $\Delta=\left\{\operatorname{det}\left(n D^{2} h_{R}\left(\beta_{0}, \sigma\right)\right)\right\}$.

Também podemos usar outros métodos para obter a densidade a posteriori marginal para $R$, como por exemplo a aproximação de Tierney, Kass e Kadane (1989), a aproximação de Leonard, Hsu e Tsui (1989) e os métodos numéricos descritos no capítulo 2.

Usando a densidade a posteriori marginal para $R$ dada em (5.31), é possível fazer inferência sobre $R$. Por exemplo, é possível construir intervalos de credibilidade para $R$, ou ainda, determinar a moda a posteriori da densidade (5.31), onde a moda é um estimador Bayesiano para o parâmetro de interesse.

Podemos obter outras estimativas Bayesianas considerando funções de perda para o parâmetro de interesse. Uma função de perda muito explorada é a função de perda quadrática dada por:

$$
G(R)=(R-\hat{R})^{2},
$$

onde $\hat{R}$ é o estimador que minimiza a perda esperada com relação à densidade a posteriori.

Considerando a função de perda quadrática, podemos mostrar facilmente que o estimador Bayesiano para o parâmetro de interesse é dado pelo valor esperado da densidade a posteriori.

Como os estimadores Bayesianos não são possíveis de se obter analiticamente, devemos recorrer a métodos numéricos ou de aproximação, como por exemplo o método de Laplace ( ver capítulo 2). 
Considerando a transformação de Guerrero e Johnson dada em (3.10) e com $\lambda$ obtido através de métodos gráficos ou métodos iterativos descritos anteriormente, a densidade a posteriori conjunta para $\phi_{G J}(\lambda), \beta_{0}$ e $\sigma$, assumindo uma priori nãoinformativa de Jeffreys, é dada por:

$$
\begin{aligned}
\pi\left(\phi_{G J}, \beta_{0}, \sigma \mid D\right) \propto & {\left[B\left(\phi_{G J}\right)\right]^{m_{2}-1}\left[1+A\left(\phi_{G J}\right)\right] \sigma^{-n}\left(1+\phi_{G J}\right)^{-1 / \lambda-1} \times \exp \left\{\sum_{i=1}^{m_{1}}\left(\frac{y_{1 i}-\beta_{0}}{\sigma}\right)+\right.} \\
+ & \left.\sum_{i=1}^{m_{2}}\left(\frac{y_{2 i}-y^{*}}{\sigma}\right)-\sum_{i=1}^{m_{1}} \exp \left(\frac{y_{1 i}-\beta_{0}}{\sigma}\right)-B\left(\phi_{G J}\right) \sum_{i=1}^{m_{2}} \exp \left(\frac{y_{2 i}-y^{*}}{\sigma}\right)\right\}
\end{aligned}
$$

onde, $A\left(\phi_{G J}\right)=\left(1+\phi_{G J}\right)^{-1 / \lambda}$ e $B\left(\phi_{G J}\right)=\ln \left[1+A\left(\phi_{G J}\right)\right]$.

A densidade a posteriori marginal para $\phi_{G J}(\lambda)$, é dada por:

$$
\begin{aligned}
\pi\left(\phi_{G J} \mid D\right) \propto & \frac{\left[B\left(\phi_{G J}\right)\right]^{n_{2}-1}}{1+A\left(\phi_{G J}\right)}\left(\phi_{G J}+1\right)^{-1 / \lambda-1} \iint \sigma^{-n} \exp \left\{\sum_{i=1}^{m_{1}}\left(\frac{y_{1 i}-\beta_{0}}{\sigma}\right)+\sum_{i=1}^{m_{2}}\left(\frac{y_{2 i}-y^{*}}{\sigma}\right)-\right. \\
& \left.-\sum_{i=1}^{n_{1}} \exp \left(\frac{y_{1 i}-\beta_{0}}{\sigma}\right)-B\left(\phi_{G J}\right) \sum_{i=1}^{n_{2}} \exp \left(\frac{y_{2 i}-y^{*}}{\sigma}\right)\right\} d \beta_{0} d \sigma .
\end{aligned}
$$

A densidade $\pi\left(\phi_{G J} \mid D\right)$ reescrita de forma adequada é dada por:

$$
\pi\left(\phi_{G J} \mid D\right) \propto \frac{\left[B\left(\phi_{G J}\right)\right]^{m_{2}-1}}{1+A\left(\phi_{G J}\right)}\left(\phi_{G J}+1\right)^{-1 / \lambda-1} \iint e^{-n h_{\phi_{G}}\left(\beta_{0}, \sigma\right)} d \beta_{0} d \sigma
$$

onde,

$$
-n h_{i \sigma}\left(\beta_{0}, \sigma\right)=-n \ln \sigma+\sum_{i=1}^{n_{1}}\left(\frac{y_{1 i}-\beta_{0}}{\sigma}\right)+\sum_{i=1}^{m_{2}}\left(\frac{y_{2 i}-y^{*}}{\sigma}\right)-\sum_{i=1}^{m_{1}} \exp \left(\frac{y_{1 i}-\beta_{0}}{\sigma}\right)-B\left(\phi_{\omega j}\right) \sum_{i=1}^{m_{2}} \exp \left(\frac{y_{2 i}-y^{*}}{\sigma}\right)
$$


Assim, a densidade a posteriori marginal aproximada para $\phi_{G J}(\lambda)$ obtida pelo método de Laplace é dada por:

$$
\pi\left(\phi_{G J} \mid D\right) \propto \frac{\left[B\left(\phi_{G J}\right)\right]^{\eta_{2-1}}}{\left[1+A\left(\phi_{G J}\right)\right] \Delta^{1 / 2}}\left(\phi_{G J}+1\right)^{-1 / \lambda} \exp \left\{-n h_{\phi_{G}}\left(\hat{\beta}_{0}, \hat{\sigma}\right)\right\}
$$

onde, $0 \leq \phi_{G J} \leq 1, \Delta=\left\{\operatorname{det} n D^{2} h_{\phi_{G J}}\left(\hat{\beta}_{0}, \hat{\sigma}\right)\right\}$ e $-n h_{\phi_{G J}}\left(\hat{\beta}_{0}, \hat{\sigma}\right)$ dado acima é calculado $\operatorname{em}\left(\hat{\beta}_{0}, \hat{\sigma}\right)$.

Para encontrar a densidade a posteriori marginal de $\phi_{G J}(\lambda)$ também poderíamos usar outros métodos de aproximações de integrais ou métodos numéricos descritos no capítulo 2 .

Usando a densidade a posteriori marginal para $\phi_{G J}(\lambda)$ dada em (5.34), é possível fazer inferências sobre o parâmetro de interesse. Por exemplo, é possível construir intervalos HPD ou ainda determinar a moda a posteriori da densidade (5.34) e seu valor esperado. 


\section{CAPÍTULO 6}

\section{EXEMPLOS DE APLICAÇÃO}

Após apresentarmos nos capitulos anteriores as idéias sobre o uso de métodos Bayesianos em modelos de regressão com dados de sobrevivência, vamos apresentar a título de ilustração alguns exemplos de aplicação das técnicas obtidas.

\subsection{Um Exemplo com o Modelo de Regressão de Feigl e Zelen}

Os resultados obtidos no capítulo 4 referentes às análises clássica e Bayesiana são aplicados neste primeiro exemplo em um conjunto de dados (ver tabela II) sob um teste de sobrevivência de pacientes com leucemia introduzido por Feigl e Zelen (1965). Esses dados são supostos terem distribuição exponencial (4.1), e a relação entre o parâmetro da distribuição de vida $\lambda$ e a covariável $x$ é dada pelo modelo de regressão de Feigl e Zelen (4.2). 


\subsubsection{Análise Clássica}

Nesta seção, aplicamos aos dados da tabela II, a metodologia de análise clássica baseada na aproximação normal assintótica dos estimadores de máxima verossimilhança dos parâmetros envolvidos (ver capítulo 4).

Consideramos o modelo introduzido em (4.1) onde $x$ é o logaritmo natural da contagem de glóbulos brancos (WBC), medida em unidades de $10000, \theta_{1}$ representa o tempo médio de um paciente com a contagem de globulos brancos de 10000 unidades e $\theta_{2}$ representa o ganho no tempo médio de sobrevivência correspondendo a um acréscimo no percentual da contagem de células de glóbulos brancos. As amostras consistem de pacientes classificados como AG positivo ou AG negativo baseado no exame das células com leucemia.

Na tabela II, temos os dados de 17 pacientes com AG positivo. A função de sobrevivência para dois anos ( $t=96$ semana ) em pacientes com a contagem de glóbulos brancos em unidade de 50.000 (ver 4.3) é dada por:

$$
R=g\left(\theta_{1}, \theta_{2}\right)=\exp \left\{-\frac{96}{\theta_{1} 5^{\theta_{2}}}\right\}
$$

A função de verossimilhança para $\theta_{1}$ e $\theta_{2}$ (ver (4.4)) é dada por:

$$
L\left(\theta_{1}, \theta_{2}\right)=\theta_{1}^{-17} \exp \left\{-3.7552 \theta_{2}-\frac{1}{\theta_{1}} \sum_{i=1}^{17} t_{i} e^{-\theta_{2} x_{i}}\right\} .
$$


Tabela II: Dados de Feigl e Zelen (AG positivo)

\begin{tabular}{|c|c|}
\hline WBC/10.000 & $\begin{array}{c}\text { Tempo de Sobrevivência } \\
\text { (em Semanas) }\end{array}$ \\
\hline 0.230 & 65 \\
0.075 & 156 \\
0.430 & 100 \\
0.600 & 134 \\
1.050 & 16 \\
1.000 & 108 \\
1.700 & 121 \\
0.540 & 4 \\
0.700 & 39 \\
0.940 & 143 \\
3.200 & 56 \\
3.500 & 26 \\
10.000 & 22 \\
10.000 & 1 \\
5.200 & 1 \\
10.000 & 5 \\
& 65 \\
\hline
\end{tabular}

Como já foi citado em capitulos anteriores, em geral os pesquisadores têm interesse em inferências sobre a função de sobrevivência $R$. Assim considerando a transformação (4.16) a função de verossimilhança para $R$ e $\phi_{2}$ é dada por:

$$
L\left(R, \phi_{2}\right)=\left[\frac{96}{(-\ln R) 5^{\phi_{2}}}\right]^{-17} \exp \left\{-3.7552 \phi_{2}+\frac{\ln R}{96} \sum_{i=1}^{n} t_{i}\left(5 e^{-x_{i}}\right)^{\phi_{2}}\right\}
$$

O logaritmo da função de verossimilhança para $R$ e $\phi_{2}$ é dado por: 


$$
\ell\left(R, \phi_{2}\right)=-77.59392+17 \ln (-\ln R)+23.60525 \phi_{2}+\frac{\ln R}{96} \sum_{i=1}^{n} t_{i}\left(5 e^{-x_{i}}\right)^{\phi_{2}}(6.4)
$$

Os estimadores de máxima verossimilhança para $R$ e $\phi_{2}$ são dados por: $\hat{R}=0.02555$ e $\hat{\phi}_{2}=-0.48183$, respectivamente. Considerando a "normalidade" assintótica dos estimadores de máxima verossimilhança para $R$ e $\phi_{2}$ baseada na matriz de informação de Fisher, os intervalos de confiança $95 \%$ para $R$ e $\phi_{2}$ são $i c(R)=(-0.01899 ; 0.07009)$ e $i c\left(\phi_{2}\right)=(-0.72316 ;-0.23634)$, onde observamos que o intervalo de confiança para $R$ está fora do limite de variação de $R$. Como mencionado no capítulo 3, a aproximação assintótica, em geral, pode não ter boa precisão. Assim na seção seguinte faremos uma análise para verificarmos a "normalidade" da função de verossimilhança de interesse.

\subsubsection{Verificação da Normalidade da Função de Verossimilhança}

Um modo simples para verificarmos a "normalidade" da função de verossimilhança é através de gráficos univariados e contornos no caso bivariado. No caso bivariado, concluimos pela "normalidade" quando encontramos contornos com formas aproximadas de elipses. Em situações quando temos interesse num dado parâmetro e existem vários parâmetros pertubadores "nuisance parameters", podemos, como uma alternativa, considerar a verossimilhança "profile" e verificar sua normalidade através da simetria dos gráficos ou usando um gráfico "t-plot" sugerido por Hills e Smith (1993) (ver capítulo 3). Também podemos verificar a "normalidade" através da derivada terceira padronizada (ver 3.7). 
Considere a função de verossimilhança padronizada $L^{*}\left(R, \phi_{2}\right)$ (ver Box $\mathrm{e}$ Tiao,1973) dada por:

$$
L^{*}\left(R, \phi_{2}\right)=\frac{\ell\left(R, \phi_{2}\right)}{\iint \ell\left(R, \phi_{2}\right) d R d \phi_{2}}
$$

Uma análise gráfica do contorno da função de verossimilhança padronizada $L^{*}\left(R, \phi_{2}\right)$ mostra que a "normalidade" não é razoável (ver figura 6.3). O contorno não se apresenta numa forma elíptica, indicando que para este caso a aproximação normal para os estimadores $\hat{R}$ e $\hat{\phi}_{2}$ não é apropriada.

Considerando o parâmetro de interesse a função de sobrevivência $R$ em $t=96$ semanas, o logaritmo da função de verossimilhança "profile" (ver Box e Tiao, 1973) para $R$, é dado por:

$$
\ell_{\hat{\phi}_{2}}(R) \propto 17 \ln (-\ln R)+17 \hat{\phi}_{2}(\ln 5-\bar{x})-\frac{\ln R}{96} \sum_{i=1}^{17} t_{i}\left(5 e^{-x_{i}}\right)^{\hat{\phi}_{2}}
$$

onde $0 \leq \mathrm{R} \leq 1$. A moda da função de verossimilhança para $R$ é dada por $\tilde{R} \cong 0.02800$ (ver figura 6.4). Na figura 6.4 observamos claramente uma forma assimétrica para a função de verossimilhança "profile" $L_{\hat{\phi}_{2}}(R)$.

Assumindo $\phi_{2}=-0.48183$ conhecido, na figura (6.1) temos o gráfico da função de verossimilhança $L(R)$ onde observamos também uma curva muito assimétrica um pouco mais fechada do que a verossimilhança "profile", significando que a verossimilhança com $\phi_{2}$ conhecido contém menos informação que a verossimilhança "profile" (ver figura 6.4). 


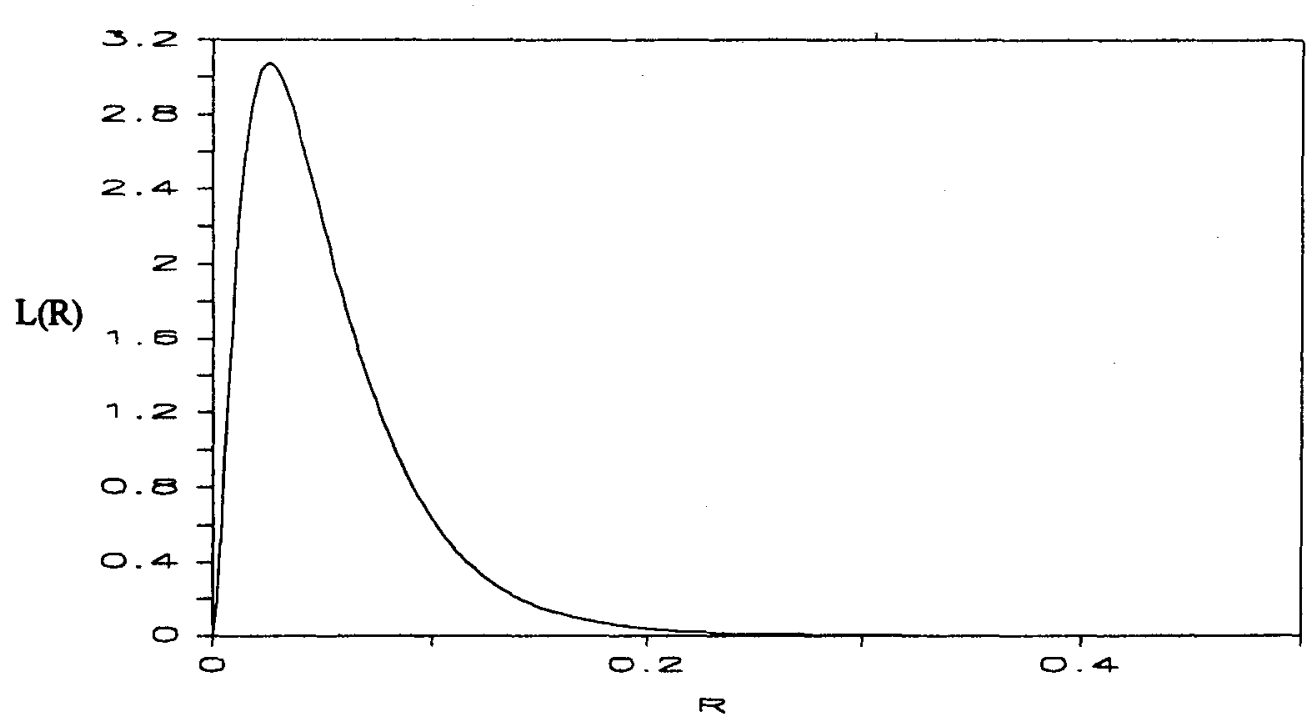

Figura 6.1: Gráfico da verossimilhança para $R \operatorname{com} \phi_{2}$ conhecido

Considerando os estimadores de máxima verossimilhança $\hat{R}=0.0255$ e $\hat{\phi}_{2}=-0.48183$ para $\mathrm{R}$ e $\phi_{2}$, a derivada terceira padronizada do logaritmo da função de verossimilhança (ver 3.7), localmente na estimativa de máxima verossimilhança $\hat{R}$ é dada por:

$$
\operatorname{STD}(\hat{R})=\left|n^{-1 / 2}(2+3 \ln \hat{R})\right|=2.1831
$$

onde observamos um valor bem diferente de zero da derivada terceira padronizada indicando que a "normalidade" não é boa.

Também podemos verificar a "normalidade" da função de verossimilhança "profile" $L_{\hat{\phi}_{2}}(R)$ usando o " $t$-plot" de Hills e Smith (1993) dado em (3.14), onde o gráfico de $T^{*}(R)$ contra alguns valores de $R$ na figura 6.5 mostra claramente uma forma curvilínea, isto é, a aproximação normal para a função de verossimilhança não é boa e $T^{*}(R)$ é dado por: 


$$
T^{*}(R)=\operatorname{sgn}(R-0.0255)\{-34 \ln (-\ln R)-10.180+9.27141(-\ln R)\}^{1 / 2}(6.9
$$

Através dos gráficos $6.1,6.2,6.3,6.5$ e da derivada terceira padronizada concluimos que a função de verossimilhança (6.3) não tem uma boa "normalidade". Para melhorar a "normalidade" consideramos a reparametrização de Guerrero e Johnson (ver 3.1) onde o logaritmo da função de verossimilhança "profile" (6.7) é dado por:

$$
\ell_{\hat{\phi}_{2}}\left(\phi_{G J}\right) \propto 17 \ln \left\{\ln \left[1+\left(\phi_{G J}+1\right)^{-1 / \lambda}\right]\right\}-\frac{\left\{\ln \left[1+\left(\phi_{G J}+1\right)^{-1 / \lambda}\right]\right\}}{96} \sum_{i=1}^{n} t_{i}\left(5 e^{-x_{i}}\right)^{\hat{\phi}_{2}} .
$$

Para determinar o valor de $\lambda$ mais apropriado que forneça boa "normalidade" para a função de verossimilhança, utilizamos a derivada terceira padronizada do logaritmo da função de verossimilhança (6.10) tal que,

$$
S T D\left(\hat{\phi}_{G J}\right)=\left|n^{-1 / 2}\left(2-3 B\left(\hat{\phi}_{G J}\right) B^{\prime \prime}\left(\hat{\phi}_{G J}\right) / B^{\prime}\left(\hat{\phi}_{G J}\right)^{2}\right)\right| \cong 0,
$$

onde $\hat{\phi}_{G J}=-0.42422$ e $B\left(\hat{\phi}_{G J}\right)=\ln \left[1+\left(\hat{\phi}_{G J}+1\right)^{-1 / \lambda}\right]($ ver Apêndice (A.1)).

Assim encontramos 0 valor de $\lambda=0.1516$ através do gráfico de $g(\lambda)=B\left(\hat{\phi}_{G J}\right) B^{\prime \prime}\left(\hat{\phi}_{G J}\right) /\left[B^{\prime}\left(\hat{\phi}_{G J}\right)\right]^{2}-2 / 3$ versus $\lambda$ (ver figura 6.2). 


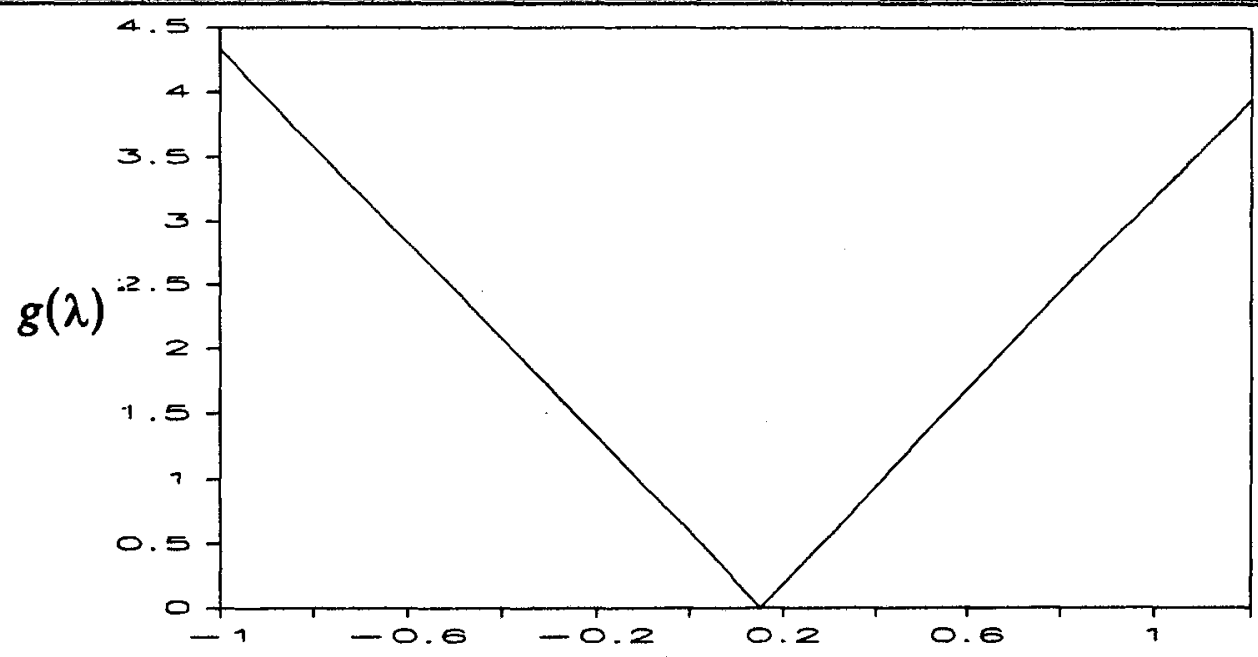

$\lambda$

Figura 6.2: Gráfico de $g(\lambda)$ versus $\lambda$

Com o valor de $\lambda=0.1516$ observamos na figura 6.4 uma grande melhoria na "normalidade" da função de verossimilhança "profile" $L_{\hat{\phi}_{2}}\left(\phi_{G J}\right)$. A moda da função de verossimilhança para $\phi_{G J}(0.1516)$ é dada por $\tilde{\phi}_{G J} \cong-0.429$.

Considerando a "normalidade" assintótica para o estimador de máxima verossimilhança $\hat{\phi}_{G J}(0.1516)=-0.42422$, encontramos um intervalo com $95 \%$ de confiança para $\phi_{G J}$ dado por $(-0,58037$; 0,26806$)$, que corresponde a um intervalo de $95 \%$ de confiança para $R$ dado por $(0,00324 ; 0,11321)$ que é um intervalo mais preciso do que o intervalo de confiança assintótico baseado na parametrização original, pois temos um intervalo de valores positivos para $R$.

Também podemos verificar a "normalidade" da verossimilhança na parametrização $\phi_{G J}$, considerando o "t-plot" proposto por Hills e Smith (1993) de $T^{*}\left(\phi_{G J}\right)$ contra alguns valores de $\phi_{G J}$, tal que,

$T^{*}\left(\phi_{G J}\right)=\operatorname{sgn}\left(\phi_{G J}+0.4242\right)\left\{-34 \ln B\left(\phi_{G J}\right)+10.1805+9.27141 \ln B\left(\phi_{G J}\right)\right\}^{1 / 2}$

onde $B\left(\phi_{G J}\right)=\ln \left(1+\left(\phi_{G J}+1\right)^{-6.59631}\right)$. 
Na figura (6.5) observamos uma melhor linearidade na parametrização $\phi_{G J}(\lambda)$, o que indica boa "normalidade" da função de verossimilhança $L_{\hat{\phi}_{2}}\left(\phi_{G J}\right)$.

$\mathrm{Na}$ figura 6.3 temos o gráfico do contorno da verossimilhança para $\phi_{2} \mathrm{e}$ $\phi_{G J}(\lambda)$ onde observamos a existência de uma boa forma elíptica, o que indica boa "normalidade" da função de verossimilhança conjunta para $\phi_{2}$ e $\phi_{G J}(\lambda)$ (ver figura 6.1).
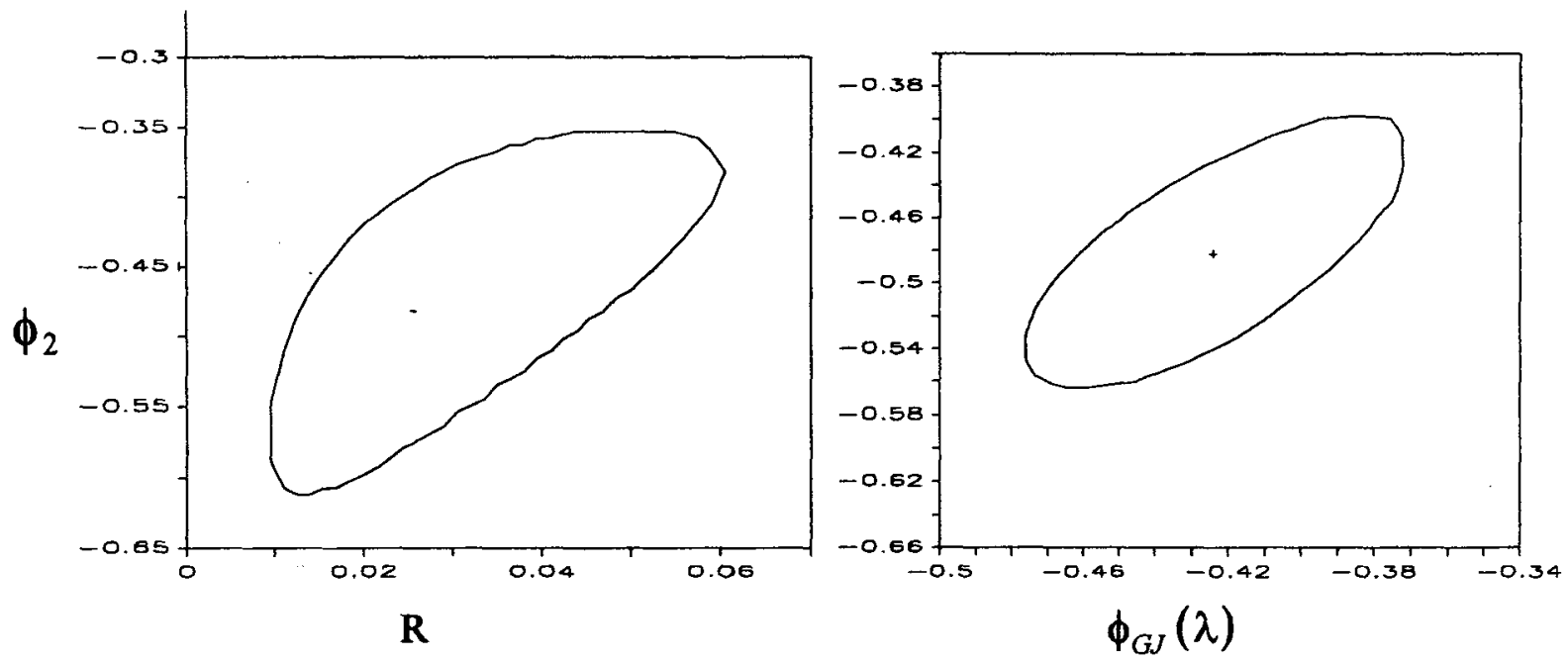

Figura 6.3: Contorno da verossimilhança padronizada para $\left(R, \phi_{2}\right)$ e $\left(\phi_{G J}(\lambda), \phi_{2}\right)$
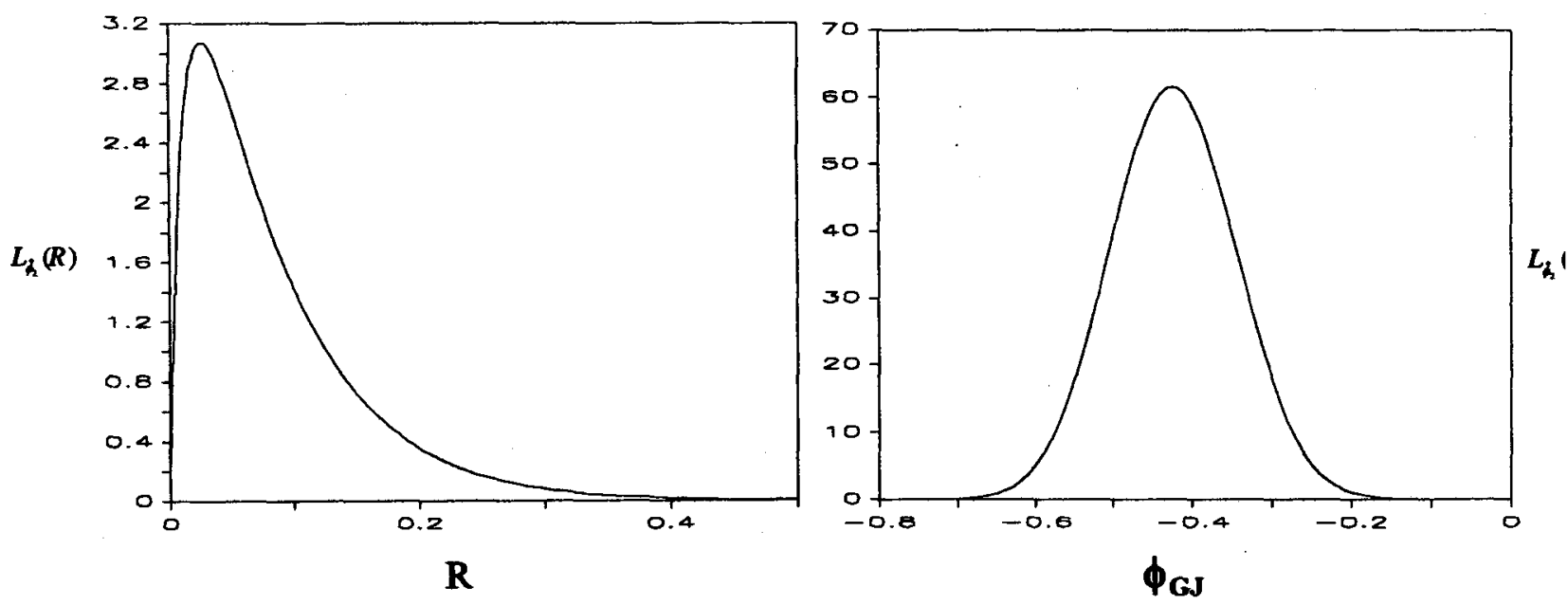

Figura 6.4: Gráfico da função de verossimilhança "profile" para $R$ e $\phi_{G J}$ 


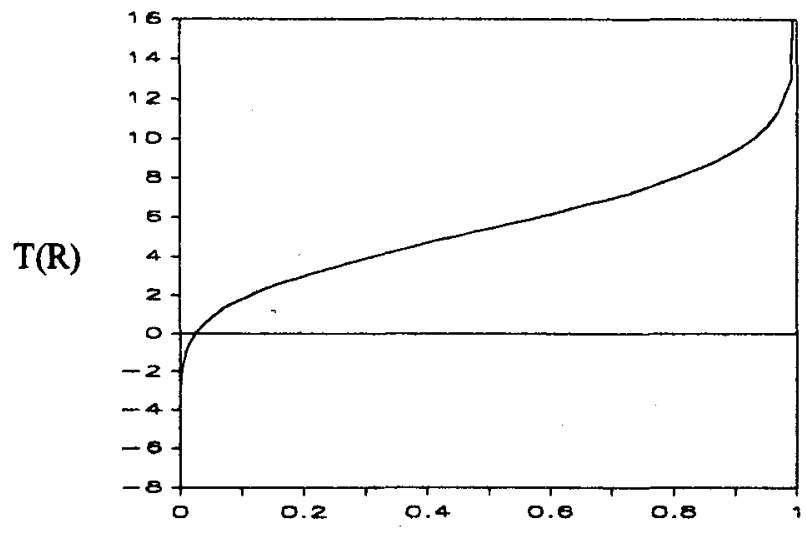

$\mathbf{R}$

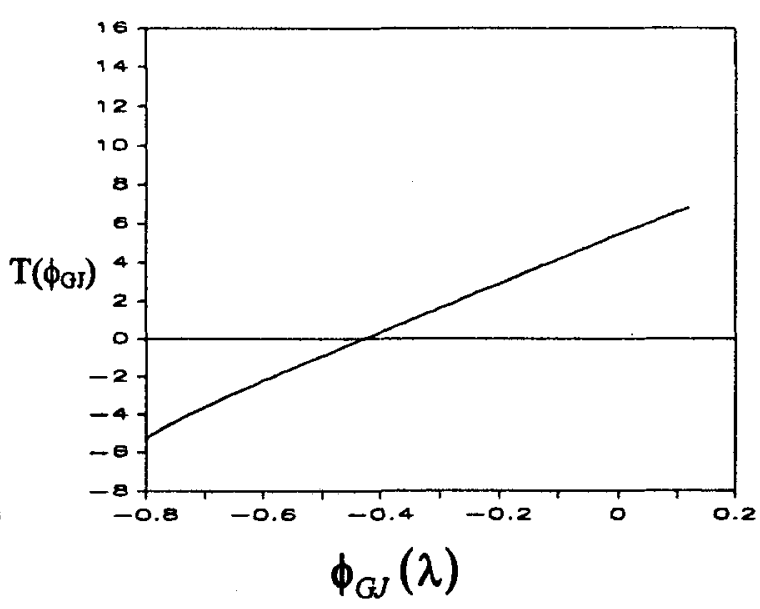

$\phi_{G J}(\lambda)$

Figura 6.5: Gráfico "t-plot" de Hills e Smith para $\mathrm{R}$ e $\phi_{G J}(\lambda)$

Também verificamos a "normalidade" da função de verossimilhança conjunta $L\left(R, \phi_{2}\right)$ e $L\left(\phi_{G J}(\lambda), \phi_{2}\right)$ usando a derivada terceira sumária (ver capítulo 3 ), onde encontramos os seguintes resultados: $m^{2} \bar{B}^{2}\left(\phi_{2}, R\right)=11.560 \quad \mathrm{e}$ $m^{2} \bar{B}^{2}\left(\phi_{2}, \phi_{G J}\right)=0.08246$. Os resultados obtidos da derivada terceira sumária confirmam os obtidos através dos gráficos de contorno (ver figura 6.3), isto é, observamos uma melhoria da "normalidade" da função de verossimilhança conjunta $L\left(\phi_{G J}(\lambda), \phi_{2}\right)$.

Assim, através dos gráficos e das medidas de não-normalidade proposta por Kass e Slate (1992) observamos a eficiência da parametrização $\phi_{G J}$ na melhoria da "normalidade" da função de verossimilhança, o que nos leva a concluir que a função de verossimilhança $L_{\hat{\phi}_{2}}\left(\phi_{G J}\right)$ tem uma boa "normalidade".

\subsubsection{Uma Solução Bayesiana}

Como visto no capítulo 4, inferências usando a metodologia Bayesiana sobre o parâmetro de interesse são baseadas nas suas densidades a posteriori, levando a 
um procedimento que pode simplificar as interpretações e conclusões para vários problemas práticos. Podemos considerar a densidade a posteriori marginal $\pi(R \mid D)$, encontrada, utilizando os métodos de aproximação de integrais ou métodos numéri$\cos$ (ver Capítulo 2) para inferência sobre a função de sobrevivência $R$. Assim temos para os dados da tabela 2 a posteriori marginal para $R$, aproximada pelo método de Laplace (ver 4.28) dada por:

$$
\pi(R \mid D) \propto \frac{(-\ln R)^{155}\left(5^{\hat{\phi}_{2}}\right)^{17}}{R\left\{\sum_{i=1}^{17} t_{i}\left(5 e^{-x_{i}}\right)^{\hat{\phi}_{2}}\left[\ln \left(5 e^{-x_{i}}\right)\right]^{2}\right\}^{1 / 2}} \exp \left\{-3.7552 \hat{\phi}_{2}+\frac{\ln R}{96} \sum_{i=1}^{17} t_{i}\left(5 e^{-x_{i}}\right)^{\hat{\phi}_{2}}\right\} .
$$

onde $0 \leq R \leq 1 \mathrm{e} \hat{\phi}_{2}$ maximiza $-n h_{R}\left(\phi_{2}\right)($ ver 4.27).

Usando a aproximação de Tierney, Kass e Kadane (1989) para funções nãolineares (ver 2.21), a densidade a posteriori marginal para $R$ é dada por:

$$
\pi_{T K K}(R \mid D) \propto \pi\left(\hat{\theta}_{1}(R), \hat{\theta}_{2}(R)\right)\left\{\frac{\operatorname{det} Z(R)}{\operatorname{det}\left[\left(D_{g}\right)^{\prime} Z(R)\left(D_{g}\right)\right]}\right\}^{1 / 2}
$$

onde $0 \leq R \leq 1, \hat{\Theta}(R)=\left(\hat{\theta}_{1}(R), \hat{\theta}_{2}(R)\right)$ maximiza $-n h\left(\theta_{1}, \theta_{2}\right)$, onde $-n h\left(\theta_{1}, \theta_{2}\right)=-18 \ln \theta_{1}-3.7552 \theta_{2}-\frac{1}{\theta_{1}} \sum_{i=1}^{n} t_{i} e^{-\theta_{2} x_{i}}$ sujeita a restrição $R=\exp \left\{-96 / \theta_{1} 5^{\theta_{2}}\right\}$.

Assim a aproximação TKK (6.13) é dada por: 


$$
\hat{\pi}_{T K K}(R \mid D) \propto \frac{(-\ln R)^{155}\left(5^{\hat{\theta}_{2}}\right)^{18}}{\left\{b_{1}^{2} a_{3}-2 b_{1} b_{2} a_{2}+a_{1} b_{2}^{2}\right\}^{1 / 2}} \exp \left\{-3.7552 \hat{\theta}_{2}+\frac{\ln R}{96} \sum_{i=1}^{17} t_{i}\left(5 e^{-x_{i}}\right)^{\hat{\phi}_{2}}\right\}(6
$$

$$
\begin{aligned}
& \text { onde, } a_{1}=\left.\frac{\partial^{2} n h}{\partial \theta_{1}^{2}}\right|_{\hat{\theta}(R)}=-\frac{(n+1)}{\hat{\theta}_{1}^{2}}+\frac{2}{\hat{\theta}_{1}^{3}} \sum_{i=1}^{n} t_{i} e^{-\hat{\theta}_{2} x_{i}}, a_{2}=\left.\frac{\partial^{2} n h}{\partial \theta_{2}^{2}}\right|_{\hat{\theta}(R)}=\frac{1}{\hat{\theta}_{1}^{2}} \sum_{i=1}^{n} t_{i} x_{i} e^{-\hat{\theta}_{2} x_{i}} \\
& a_{3}=\left.\frac{\partial^{2} n h}{\partial \theta_{2}^{2}}\right|_{\hat{\theta}(R)}=\frac{1}{\hat{\theta}_{1}} \sum_{i=1}^{n} x_{i}^{2} t_{i} e^{-\hat{\theta}_{2}}, b_{1}=\frac{R(-\ln R)}{\hat{\theta}_{1}} \text { e } b_{2}=R(-\ln R) \ln 5 .
\end{aligned}
$$

Também podemos considerar a aproximação de Leonard, Hsu e Tsui (1989) (ver 2.22), então a densidade a posteriori marginal para $R$ é dado por:

$$
\begin{aligned}
\hat{\pi}_{L H T}(R \mid D) \propto \Delta^{-1 / 2} \hat{\theta}_{1}^{-18} \exp \left\{-3.7552 \hat{\theta}_{2}+-\frac{1}{\hat{\theta}_{1}} \sum_{i=1}^{n} t_{i} e^{-\hat{\theta}_{2} x_{i}}\right\} \times \\
\quad \times \exp \left\{\frac{1}{2 \Delta}\left[d_{1}^{2} c_{3}-2 d_{1} d_{2} c_{3}+c_{1} d_{2}^{2}\right]\right\} \frac{1}{\sqrt{2 \pi} \sigma} \exp \left\{-\frac{1}{2 \sigma^{2}}(\eta-\bar{\eta})^{2}\right\}
\end{aligned}
$$

onde $\quad \Delta=a_{1} a_{3}-a_{2}^{2}, \quad \sigma^{2}=\left[D_{g} \tilde{\theta}(R)\right]^{\prime} \mathbb{Z}(R)\left[D_{g} \tilde{\theta}(R)\right]=\frac{1}{\Delta}\left(c_{1}^{2} a_{3}-2 c_{1} c_{2} a_{2}+a_{1} c_{2}^{2}\right)$ $\left(c_{1}=b_{1}, c_{2}=b_{2}, a_{1}, a_{2}\right.$ e $a_{3}$ são dados em 6.14) e $\eta=\exp \left\{-96 / \tilde{\theta}_{1}(R) 5^{\tilde{\theta}_{2}(R)}\right\}$.

$\mathrm{Na}$ figura 6.6 temos o gráfico da densidade a posteriori marginal (6.12), (6.14), (6.15) e também a densidade obtida usando o método numérico de Gauss Hermite, onde as modas dessas densidades a posteriori são dadas por $\tilde{R} \cong 0.00999$, $\tilde{R} \cong 0.00499 \tilde{R} \cong 0.01499$ e $\tilde{R} \cong 0.03999$ respectivamente.

Observamos que as densidades a posteriori obtidas utilizando os métodos de aproximação para integrais e os métodos numéricos não têm uma forma bem comportada (aproximadamente normal), onde pode ter sua precisão comprometida. Assim a procura de uma parametrização adequada torna-se interessante. 


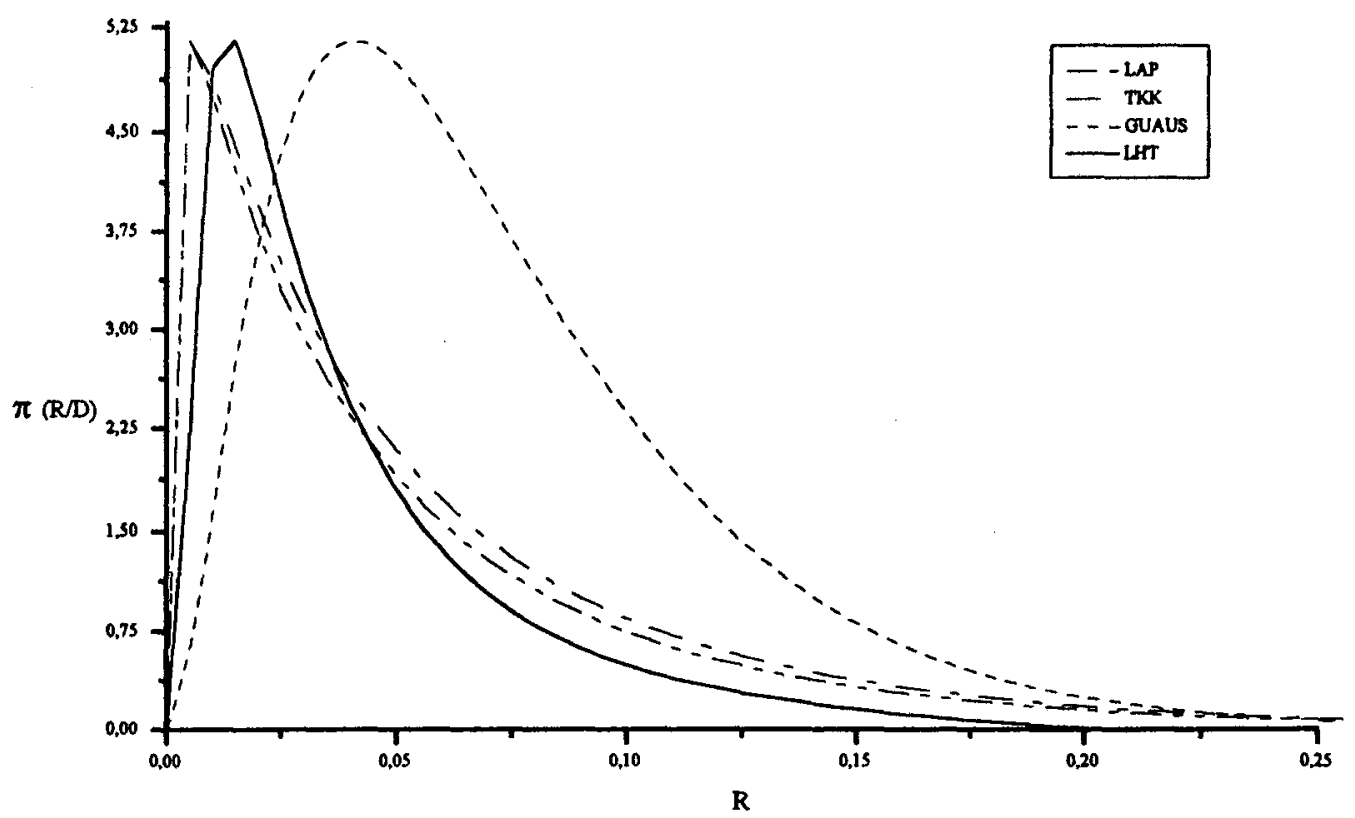

Figura 6.6 Densidade a posteriori marginal para $R$

Considerando a transformação de Guerrero e Johnson com $\lambda=0.1516$, a densidade a posteriori conjunta para $\phi_{G J}(\lambda)$ e $\phi_{2}$ (ver 4.31) é dada por:

$$
\begin{gathered}
\pi\left(\phi_{G J}, \phi_{2} \mid D\right) \propto \frac{\left[\ln \left(1+\left(\phi_{G J}+1\right)^{-6.59631}\right)\right]^{16}}{1+\left(\phi_{G J}+1\right)^{-6.59631}} 5^{17 \phi_{2}}\left(\phi_{G J}+1\right)^{-7.59631} \times \\
\times \exp \left\{-3.7552 \phi_{2}-\frac{\ln \left[1+\left(\phi_{G J}+1\right)^{-6.59631}\right]}{96}\right\} \sum_{i=1}^{17} t_{i}\left(5 e^{-x_{i}}\right)^{\phi_{2}}, \quad \text { (6.16) }
\end{gathered}
$$

onde, $-1<\phi_{G J}<\infty$ e $-\infty<\phi_{2}<\infty$.

Para inferências sobre o parâmetro $\phi_{G J}(\lambda)$ considerando o conjunto de dados da tabela II, contruímos o gráfico da densidade a posteriori marginal aproximada para $\phi_{G J}(\lambda)$, obtida pelos métodos de aproximações de integrais e pelo método numérico (ver capítulo 2). Usando o método de Laplace obtemos a densidade a posteriori marginal para $\phi_{G J}($ ver 4.34$)$ dada por: 


$$
\begin{aligned}
& \hat{\pi}\left(\phi_{G J} \mid D\right) \propto \frac{\left[\ln \left(1+\left(\phi_{G J}+1\right)^{-6.59631}\right)\right]^{155}\left(5^{\hat{\phi}_{2}}\right)^{17}\left(\phi_{G J}+1\right)^{-7.59631}}{\left[1+\left(\phi_{G J}+1\right)^{-6.59631}\right]\left\{\sum_{i=1}^{17} t_{i}\left(5 e^{-x_{i}}\right)^{\hat{\phi}_{2}}\left[\ln \left(5 e^{-x_{i}}\right)\right]^{2}\right\}^{1 / 2}} \\
& \times \exp \left\{-3.7552 \hat{\phi}_{2}-\frac{\ln \left[1+\left(\phi_{G J}+1\right)^{6.59631}\right]}{96} \sum_{i=1}^{17} t_{i}\left(5 e^{-x_{i}}\right)^{\hat{\phi}_{2}}\right\}
\end{aligned}
$$

onde $-1<\phi_{G J}<\infty$ e $\hat{\phi}_{2}$ é obtido usando o procedimento iterativo de NewtonRaphson para cada valor de $\phi_{G J}$.

Considerando a aproximação de Tierney, Kass e Kadane (1989) para funções não- lineares (ver 2.21), a densidade a posteriori marginal para $\phi_{G J}$ é dada por:

$$
\pi_{T K K}\left(\phi_{G J} \mid D\right) \propto e^{-n h\left(\hat{R}\left(\phi_{G J}\right), \hat{\phi}_{2}\left(\phi_{G J}\right)\right)}\left\{\frac{\operatorname{det} \Sigma\left(\phi_{G J}\right)}{\operatorname{det}\left[\left(D_{g}\right)^{\prime} \mathrm{Z}\left(\phi_{G J}\right)\left(D_{g}\right)\right]}\right\}^{1 / 2},
$$

onde $-1 \leq \phi_{G J} \leq \infty \hat{\Theta}\left(\phi_{G J}\right)=\left(\hat{R}\left(\phi_{G J}\right), \hat{\phi}_{2}\left(\phi_{G J}\right)\right)$ maximiza $-n h\left(R, \phi_{2}\right)$, onde $-n h\left(R, \phi_{2}\right)=16 \ln (-\ln R)+17 \phi_{2} \ln 5-3.7552 \phi_{2}+\frac{\ln R}{96} \sum_{i=1}^{17} t_{i}\left(5 e^{-x_{1}}\right)^{\phi_{2}}$ sujeita à restrição $\phi_{G J}=g\left(R, \phi_{2}\right)=(R / 1-R)^{\lambda}-$, isto é, $\ln \left(\ln \phi_{G J}\right)=\ln \left[\ln \left[(R / 1-R)^{\lambda}-1\right]\right]$, $\mathcal{Z}\left(\phi_{G J}\right)$ é a inversa da matriz Hessiana de $n h\left(R, \phi_{2}\right)$ em $\hat{\Theta}\left(\phi_{G J}\right)$ e $D_{g}$ é o gradiente de $g$ calculado em $\hat{\Theta}\left(\phi_{G J}\right)$.

Para maximizar $-n h\left(R, \phi_{2}\right)$ sujeita à restrição $\ln \left(\ln \phi_{G J}\right)=\ln \left[\ln \left[(R / 1-R)^{\lambda}-1\right]\right]$, consideramos a função,

$$
Q\left(R, \phi_{2}\right)=-n h\left(R, \phi_{2}\right)-\mu\left\{\ln \left(-\ln \phi_{G J}\right)-\ln \left\{\ln \left[\left(\frac{R}{1-R}\right)^{\lambda}-1\right]\right\}\right\}
$$


onde $\mu$ é o multiplicador de Lagrange.

Usando o método iterativo de Newton-Raphson, encontramos $\hat{\phi}_{2}\left(\phi_{G J}\right)$ para cada valor de $\phi_{G J}$, tal que,

$$
f\left(\phi_{2}\right)=\frac{96\left(\sum x_{i}-\ln 5\right)}{\sum_{i=1}^{17} t_{i}\left(5 e^{-x_{i}}\right)^{\phi} \ln \left(5 e^{-x_{i}}\right)}-6.59631 \ln \left(\phi_{G J}+1\right)+\ln \left(1+\left(\phi_{G J}+1\right)^{659631}\right)=0 .
$$

A inversa da matriz Hessiana para $n h\left(R, \phi_{2}\right)$ em $\hat{\Theta}\left(\phi_{G J}\right)$ é dada por:

$$
\Sigma\left(\phi_{G J}\right)=\left(\begin{array}{cc}
a_{3} / \Delta & -a_{2} / \Delta \\
-a_{2} / \Delta & a_{1} / \Delta
\end{array}\right)
$$

onde, $\Delta=a_{1} a_{3}-a_{2}^{2}$

$a_{1}=\frac{n \partial^{2} h}{\partial R^{2}} /_{\hat{\mathbf{\theta}}\left(\phi_{G)}\right)}=(n-1) / \hat{R}^{2} \ln \hat{R}+(n-1) / \hat{R}(\ln \hat{R})^{2}-1 / \hat{R}^{2}+A^{*}\left(\hat{\phi}_{2}\right) / \hat{R}^{2} 96$,

$a_{2}=-A^{*}\left(\hat{\phi}_{2}\right) / 96 \hat{R}, a_{3}=-\ln \hat{R} A^{* \prime}\left(\hat{\phi}_{2}\right) / 96$ e $A^{*}\left(\hat{\phi}_{2}\right)=\sum_{i=1}^{n} t_{i}\left(5 e^{-x_{i}}\right)^{\hat{\phi}_{2}}$ (ver Apêndice (A.1)).

De (6.21) temos $\operatorname{det} \mathcal{Z}\left(\phi_{G J}\right)=\Delta^{-1},\left(D_{g}\right)^{\prime} Z\left(\phi_{G J}\right)\left(D_{g}\right)=\Delta^{-1}\left(b_{1}^{2} a_{3}\right)$, onde $\left(D_{g}\right)^{\prime}=\left(b_{1}, 0\right), b_{1}=\frac{\partial g}{\partial R} /_{\hat{\Theta}\left(\phi_{G J}\right)}=\lambda\left(\phi_{G J}+1\right) / \hat{R}(1+\hat{R})$.

Assim a aproximação TKK (6.18) é dada por:

$$
\hat{\pi}_{T K K}\left(\phi_{G J} \mid D\right) \propto \frac{(-\ln \hat{R})^{16}\left(5 e^{\hat{\phi}_{2}}\right)^{17}}{\hat{R}\left(b_{1}^{2} a_{3}\right)^{1 / 2}} \exp \left\{-3.7552 \hat{\phi}_{2}+\frac{\ln \hat{R}}{96} \sum_{i=1}^{17} t_{i}\left(5 e^{-x_{i}}\right)^{\hat{\phi}_{2}}\right\}
$$


onde $-1 \leq \phi_{G J}<\infty$.

Outra possibilidade é considerar a aproximação de Leonard, Hsu e Tsui (1989) (ver (2.22)). O máximo de $-n h\left(R, \phi_{2}\right)$ dado em (6.18) sujeita à restrição $\phi_{G J}=g\left(R, \phi_{2}\right)=(R / 1-R)^{\lambda}-1$ é dado pelas expressões (6.19) e (6.20). O gradiente de $n h\left(R, \phi_{2}\right)$ em $\hat{\Theta}\left(\phi_{G J}\right)$ é dado por $\ell_{\phi_{J}}^{\prime}=\left(d_{1}, d_{2}\right)$, onde,

$$
d_{1}=\frac{\partial n h}{\partial R} /_{\hat{\Theta}\left(\phi_{G J}\right)}=\frac{1}{\hat{R}}\left[-\frac{(n-1)}{\ln \hat{R}}+1-\frac{A^{*}\left(\hat{\phi}_{2}\right)}{96}\right]
$$

e

$$
d_{2}=\frac{\partial n h}{\partial \phi_{2}} /_{\hat{\Theta}\left(\phi_{G J}\right)}=-n \ln 5+\sum_{i=1}^{n} x_{i}-\frac{\ln \hat{R} A^{*}\left(\hat{\phi}_{2}\right)}{96}
$$

Também, $\tilde{\Theta}\left(\phi_{G J}\right)=\left(\tilde{R}\left(\phi_{G J}\right), \tilde{\phi}_{2}\left(\phi_{G J}\right)\right)$ é dado (ver (2.23)) por $\tilde{R}\left(\phi_{G J}\right)=\hat{R}\left(\phi_{G J}\right)-\Delta^{-1}\left(a_{3} d_{1}-a_{2} d_{2}\right)$ e $\tilde{\phi}_{2}\left(\phi_{G J}\right)=\hat{\phi}\left(\phi_{G J}\right)-\Delta^{-1}\left(a_{1} d_{2}-a_{2} d_{1}\right)$ onde $d_{1}$ e $d_{2}$ são dados em (6.23) e $a_{1}, a_{2}$ e $a_{3}$ são dados em (6.22).

Considerando a aproximação normal $N(\mu, \mathbb{C})$ para $f\left(\phi_{G J} \mid \hat{\Theta}\left(\phi_{G J}\right), \mathbb{Z}\left(\phi_{G J}\right)\right)$ $(\operatorname{ver}(2.23)), \quad$ onde $\quad \mathbb{C}=\left[D_{g}\left(\tilde{\Theta}\left(\phi_{G J}\right)\right)\right]^{\prime} Z\left(\phi_{G J}\right)\left[D_{g}\left(\tilde{\Theta}\left(\phi_{G J}\right)\right)\right]=\Delta^{-1}\left(\tilde{c}_{1}^{2} a_{3}\right)$, $\tilde{c}_{1}=\partial g /\left.\partial R\right|_{\tilde{\Theta}\left(\phi_{G J}\right)}=\frac{\lambda\left(\phi_{G J}+1\right)}{\tilde{R}\left(\phi_{G J}\right)\left(1-\tilde{R}\left(\phi_{G J}\right)\right)}$ e $\mu=g\left(\tilde{\Theta}\left(\phi_{G J}\right)\right)=\left(\frac{\tilde{R}}{1-\tilde{R}}\right)^{\lambda}-$ e sendo $\ell_{\phi_{G}}^{\prime} \mathrm{Z}\left(\phi_{G J}\right) \ell_{\phi_{G J}}=\Delta^{-1}\left[d_{1}^{2} a_{3}-2 d_{1} d_{2} a_{3}+a_{1} d_{2}^{2}\right]$, temos a aproximação LHT dada por:

$$
\begin{aligned}
\hat{\pi}_{L H T}\left(\phi_{G J} \mid D\right) \propto \frac{(-\ln \hat{R})^{16}\left(5^{\hat{\phi}_{2}}\right)^{17} \exp \left\{-\frac{1}{2 C}\left(\phi_{G J}-\mu\right)^{2}\right\}}{\Delta^{1 / 2} \hat{R}} \times \\
\times \exp \left\{-3.7552 \hat{\phi}_{2}-\frac{\ln \hat{R}}{96} \sum_{i=1}^{17} t_{i}\left(5 e^{-x_{i}}\right)^{\hat{\phi}_{2}}+\frac{1}{2 \Delta}\left[d_{1}^{2} a_{3}-2 d_{1} d_{2} a_{3}+a_{1} d_{1}^{2}\right]\right\}
\end{aligned}
$$


onde $-1 \leq \phi_{G J}<\infty$.

$\mathrm{Na}$ figura 6.7 temos os gráficos das densidades a posteriori (6.17),(6.22), (6.24) e a densidade obtida usando o método numérico de Gauss Hermite, onde as modas são dadas por $\tilde{\phi}_{G J} \cong-0.4100, \tilde{\phi}_{G J} \cong-0.3100$ e $\tilde{\phi}_{G J} \cong-0.3700$ respectivamente. Observamos que as densidades obtidas pelos métodos de aproximações e o método numérico têm uma forma bem mais aproximada da normal e o método de aproximação LHT é razoavelmente próximo do obtido pelo método numérico, o que não ocorre na parametrização original, sabendo que o método numérico é o mais próximo do real podemos concluir que usando a parametrização de Guerrero e Johnson temos boas aproximações baseadas em (6.17), (6.22) e (6.24), isto é, podemos decidir por um dos métodos aplicados.

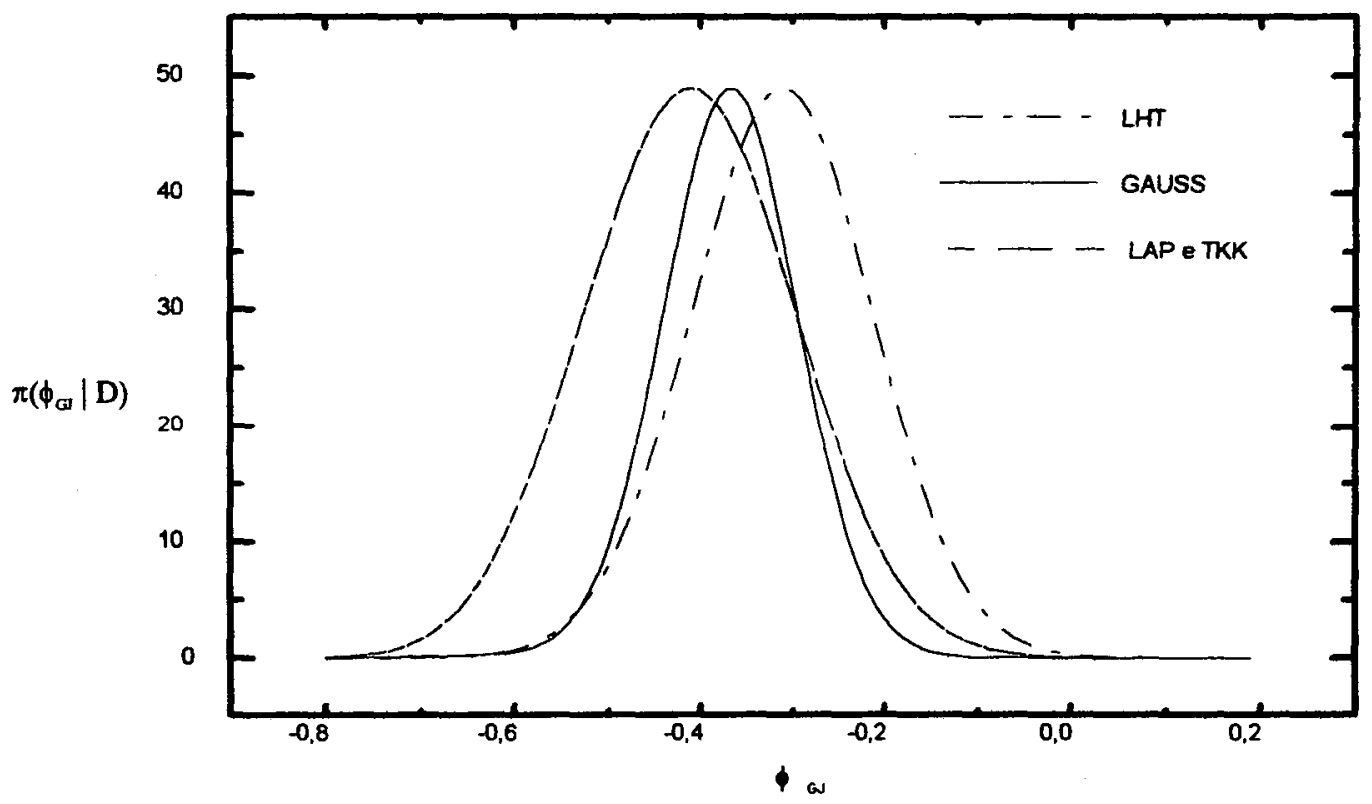

Figura 6.7: Densidade a posteriori marginal para $\phi_{G J}$ 
O intervalo HPD aproximado $95 \%$ para $\phi_{G J}$ é, $(-0.56460 ;-0.25130)$ (ver Apêndice (A.2)) o qual utilizamos a distribuição marginal obtida pelo método de Laplace (6.17). O intervalo HPD correspondente para $R$ é, $(0.00413 ; 0.12908)$.

Observamos que um intervalo de confiança $95 \%$ para $\phi_{G J}$ obtido a partir da "normalidade" assintótica do estimador de máxima verossimilhança $\hat{\phi}_{G J}$ é dado por: $(-0.58037 ; 0.26806)$. O intervalo de confiança correspondente para $R$ é, $(0.00324 ; 0.11321)$ o qual é muito próximo do intervalo HPD, entretanto, é diferente do intervalo de confiança 95\% para $R$ obtido a partir da "normalidade" assintótica do estimador de máxima verossimilhança $\hat{R}$ que é dado por, $(-0.01899 ; 0.07009)$.

Assim, observamos que na parametrização de Guerrero e Johnson temos melhor estimativa para o parâmetro $R$, sendo que o intervalo tem apenas valores positivo. Isto mostra a necessidade de utilizarmos uma parametrização adequada quando usamos resultados baseados na "normalidade" assintótica dos estimadores de máxima verossimilhança ou para a densidade a posteriori para o parâmetro de interesse, pois as inferências aproximadas na parametrização original podem ser muito ruins.

\subsection{Um Exemplo com a Distribuição de Valor Extremo}

\subsubsection{Comparação de Dois Tratamentos}

Os resultados obtidos no capítulo 5 referentes ás análises clássica e Bayesiana, são aplicados, nesta seção, em um conjunto de dados (ver tabela III) sob um teste de sobrevivência para dois grupos de tratamentos onde os dados (tempos de sobrevivências em semanas), foi introduzido por Lee (1980). O logaritmo do tempo de vida 
para os dois grupos de tratamentos são supostos terem distribuição de valor extremo (5.1) (ver por exemplo Achcar e Bolfarine, 1986).

Tabela III: Tempos de Sobrevivência de Pacientes em dois Grupos de Tratamentos

\begin{tabular}{|l|l|}
\hline Tratamento 1 (controle) & Tratamento 2 (novo tratamento) \\
\hline $5,10,17,32,32,33,34,36$, & $20.9,32.2,33.2,39.4,40.0,46.8,57.3$, \\
$43,44,44,48,48,61,64,65$, & $58.0,59.7,61.1,61.4,54.3,66.0,66.3$, \\
$65,66,67,68,82,85,90,92$, & $67.4,68.5,69.9,72.4,73.0,73.2,88.7$, \\
$92,102,103,106,107,114$, & $89.3,91.6,93.1,94.2,97.7,101.6,101.9$, \\
$114,116,117,124,139,142$, & $107.6,108.0,109.7,110.8,114.1,117.5$, \\
$143,151,158,195$ & $119.2,120.3,133.0,133.8,163.3,165.1$ \\
\hline
\end{tabular}

\subsubsection{Análise Clássica}

Nesta seção aplicamos aos dados da tabela III, a metodologia de análise clássica baseada na aproximação normal assintótica dos estimadores de máxima verossimilhança para os parâmetros envolvidos (ver capítulo 5).

Considerando os dados da tabela III do logaritmo da função de verossimilhança (5.13), obtemos numericamente os estimadores de máxima verossimilhança para $\beta_{0}, \beta_{1}$ e $\sigma$ dados por, $\hat{\beta}_{0}=4.54978, \hat{\beta}_{1}=-0.024037$ e $\hat{\sigma}=0.453073$. A matriz de informação de Fisher $I$, para $\beta_{0}, \beta_{1}$ e $\sigma$ calculada em $\left(\hat{\beta}_{0}, \hat{\beta}_{1}, \hat{\sigma}\right)$ é dada de (5.6)) por: 


$$
I\left(\beta_{0}, \beta_{1}, \sigma\right)=\left[\begin{array}{ccc}
389.721 & 194.860 & 164.768 \\
194.860 & 194.860 & 194.860 \\
164.768 & 82.384 & 321.006
\end{array}\right]
$$

Os estimadores de máxima verossimilhança têm distribuição normal assintótica:

$$
\left(\hat{\beta}_{0}, \hat{\beta}_{1}, \hat{\sigma}\right) \stackrel{a}{\sim} N\left\{\left(\beta_{0}, \beta_{1}, \sigma\right) ; I^{-1}\left(\beta_{0}, \beta_{1}, \sigma\right)\right\}
$$

onde $I^{-1}\left(\hat{\boldsymbol{\beta}}_{0}, \hat{\boldsymbol{\beta}}_{1}, \hat{\sigma}\right)$ é:

$$
I^{-1}\left(\hat{\beta}_{0}, \hat{\beta}_{1}, \hat{\sigma}\right)=\left[\begin{array}{ccc}
0.0048721 & -0.0051319 & 0.0006144 \\
0.0031900 & 0.0102638 & -0.0045930 \\
0.0016821 & 0.000000 & 0.0039786
\end{array}\right]
$$

Em trabalhos práticos é comum considerar a matriz de informação observada em lugar da matriz de informação de Fisher. Portanto o inverso da matriz de informação observada $I_{0}^{-1}\left(\hat{\beta}_{0}, \hat{\beta}_{1}, \hat{\sigma}\right)$ calculada nos estimadores de máxima verossimilhança $\hat{\beta}_{0}, \hat{\beta}_{1}$ e $\hat{\sigma}$ (ver 5.5) é:

$$
I_{0}^{-1}\left(\hat{\beta}_{0}, \hat{\beta}_{1}, \hat{\sigma}\right)=\left[\begin{array}{ccc}
0.0052098 & -0.0056134 & 0.0004036 \\
-0.0056134 & 0.0132402 & -0.0024949 \\
0.0004036 & -0.0024949 & 0.0020912
\end{array}\right]
$$

e os intervalos de confiança $95 \%$ para $\beta_{0}, \beta_{1}$ e $\sigma$, considerando-se a aproximação assintótica dos estimadores de máxima verossimilhança $\hat{\beta}_{0}, \hat{\beta}_{1}$ e $\hat{\sigma}$, estão dados na 
tabela IV. Observamos que todos os intervalos para $\beta_{0}, \beta_{1}$ e $\sigma$ são aproximadamente iguais.

Tabela IV: Intervalos de Confiança para $\beta_{0}, \beta_{1}$ e $\sigma$

\begin{tabular}{|c|c|c|}
\hline Parâmetros & $\begin{array}{c}\text { Informação observada } \\
I_{0}\left(\hat{\beta}_{0}, \hat{\beta}_{1}, \hat{\sigma}\right)\end{array}$ & $\begin{array}{c}\text { Informação de Fisher } \\
I^{-1}\left(\hat{\beta}_{0}, \hat{\beta}_{1}, \hat{\sigma}\right)\end{array}$ \\
\hline$\beta_{0}$ & {$[4.10241 ; 4.99715]$} & {$[4.54978 ; 4.68659]$} \\
\hline$\beta_{1}$ & {$[-0.24957 ; 0.20145]$} & {$[-0.22261 ; 0.17453]$} \\
\hline$\sigma$ & {$[0.36344 ; 0.54270]$} & {$[0.32944 ; 0.57670]$} \\
\hline
\end{tabular}

Testes de hipóteses para os parâmetros podem ser construidos pela distribuição normal assintótica ou pelo teste da razão de verossimilhanças. Portanto para testar as hipóteses $H_{0}: \beta_{1}=0$ contra $H_{1}: \beta_{1} \neq 0$ (ver capítulo 1) temos que, para a "normalidade" assintótica (ver 1.29) a estatística teste é dada por:

$$
\mathrm{Z}_{\text {observado }}=\frac{\hat{\beta}_{1}-\hat{\beta}_{1}}{\sqrt{\operatorname{var} \beta_{1}}}=\frac{-0.0240366}{\sqrt{0.0132402}}=-0.208
$$

e a região crítica para o nível de $5 \%$ de significância é, dada por $|Z| \geq 1.96$. Como, $\left|Z_{\text {observado }}\right|<1.96$, não rejeitamos a hipótese $H_{0}: \beta_{1}=0$ ao nível de significância de $5 \%$.

Para o teste da razão de verossimilhança (ver 1.30) os estimadores de máxima verossimilhança sob $o$ espaço com restrição $H_{0}: \beta_{1}=0$ é, $\tilde{\sigma}=0.451073$, $\tilde{\beta}_{0}=4.55076$ e $\tilde{\beta}_{1}=0$. Portanto, a estatística da razão de verossimilhanças é dada por: 


$$
\Delta_{\text {tase }}=-2 \ell\left(\tilde{\beta}_{0}, \tilde{\beta}_{1}, \tilde{\sigma}\right)+2 \ell\left(\hat{\beta}_{0}, \hat{\beta}_{1}, \hat{\sigma}\right)=0.117
$$

e a região crítica para o nível de $5 \%$ de siginificância é dada a partir da distribuição quiquadrado, por $\chi_{1(0.05)}^{2} \geq 3.84$. Como $\Delta_{\text {toste }}<\chi_{1(0.05)}^{2}$, concluimos pela não rejeição da hipótese $H_{0}: \beta_{1}=0$ ao nível de siginificância igual à $5 \%$.

Como foi mencionado em capítulos anteriores em geral o pesquisador tem interesse em inferências sobre a função de sobrevivência $R$, e a precisão dos resultados assintóticos depende da boa "normalidade" da função de verossimilhança. Portanto na seção seguinte vamos verificar a "normalidade" da função de verossimilhança (5.15).

\subsection{Verificação da Normalidade da Função de Verossimilhança}

Considerando $\beta_{0}$ e $\sigma$ conhecidos (iguais aos estimadores de máxima verossimilhança $\hat{\beta}_{0}$ e $\hat{\sigma}$ ) podemos verificar a "normalidade" da função de verossimilhança para $R$ (5.15) através da derivada terceira padronizada do logaritmo da função de verossimilhança localmente em $\hat{R}$ definido em (5.17) para o novo tratamento $\left(x^{*}=\right)$. Na tabela $\mathrm{V}$ temos os estimadores de máxima verossimilhança para $R$, e os valores de $\operatorname{STD}(\hat{R})$ considerando diferentes valores para $y^{*}=\ln \left(t_{0}\right)$. 
Tabela V: Estimador de máxima verossimilhança para $R\left(y^{*}\right) \operatorname{com} y^{*}=\ln \left(t_{0}\right)$ e valores para $S T D(\hat{R})\left(\sigma=0.4534\right.$ e $\beta_{0}=4.5496$ conhecidos $)$

\begin{tabular}{|c|c|c|}
\hline$t_{0}$ & $\hat{R}\left(y^{*}\right)$ & $S T D(\hat{R})$ \\
\hline 5 & 0.9984 & 0.3155 \\
\hline 20 & 0.9663 & 0.3000 \\
\hline 60 & 0.6795 & 0.1330 \\
\hline 80 & 0.4826 & 0.0294 \\
\hline 120 & 0.1683 & 0.5289 \\
\hline 180 & 0.0128 & 1.7507 \\
\hline 200 & 0.0041 & 2.2914 \\
\hline 300 & 0.000001 & 6.0609 \\
\hline
\end{tabular}

Observamos na tabela $\mathrm{V}$ alguns valores grandes para $S T D(\hat{R})$, especialmente para valores grandes de $t_{0}$, o qual indica "normalidade" ruim para a função de verossimilhança de $R\left(y^{*}\right)$.

Assumindo $\beta_{0}$ e $\sigma$ conhecidos, na figura 6.8 temos os gráficos da função de verossimilhança "profile" para $R\left(y^{*}\right)$ considerando os diferentes valores de $t_{0}$, definida em (5.18), onde observamos uma certa assimetria para os diferentes valores de $t_{0}$, indicando que a "normalidade" não é boa. Asim, observando a figura 6.8 e a derivada terceira padronizada dada na tabela $\mathrm{V}$, verificamos que uma reparametrização deve ser aplicada. Portanto, exploramos a parametrização de Guerrero e Johnson (ver capítulo 3 ), onde esperamos uma melhor aproximação normal dos estimadores de máxima verossimilhança de $\phi_{G J}$.

Considerando $\beta_{0}$ e $\sigma$ conhecidos, na tabela VI temos o valor de $\lambda$ apropriado que fornece uma boa "normalidade" para a função de verossimilhança (5.23). Para encontrar $\lambda$ utilizamos a derivada terceira padronizada do logaritmo da função de 
verossimilhança (de 5.25) dado por $S T D\left(\hat{\phi}_{G J}(\lambda)\right) \cong 0$. Também temos na tabela VI os estimadores de máxima verossimilhança de $\phi_{G J}(\lambda)$ e os valores de $\operatorname{STD}\left(\hat{\phi}_{G J}(\lambda)\right)$ correspondentes. Comparando os valores de $\operatorname{STD}\left(\hat{\phi}_{G J}(\lambda)\right)$ com os valores de $\operatorname{STD}(\hat{R})$ na tabela $\mathrm{V}$ podemos ver claramente que a parametrização $\phi_{G J}(\lambda)$ melhora sensivelmente a "normalidade" da função de verossimilhança.

Tabela VI: Estimador de máxima verossimilhança para $\phi_{G J}(\lambda) \operatorname{com}$

$$
y^{*}=\ln \left(t_{0}\right) \text { e valores de } S T D\left(\hat{\phi}_{G J}(\lambda)\right)
$$

\begin{tabular}{|c|c|c|c|}
\hline$t_{0}$ & $\lambda$ & $\hat{\phi}_{G J}(\lambda)$ & $S T D\left(\hat{\phi}_{G J}(\lambda)\right)$ \\
\hline 5 & -0.997330 & -0.998369 & 0.0000072 \\
\hline 20 & -0.939778 & -0.957469 & 0.0000062 \\
\hline 60 & -0.462226 & -0.293962 & 0.0000012 \\
\hline 80 & -0.287334 & 0.019991 & 0.0000004 \\
\hline 120 & -0.094779 & 0.163504 & 0.0000001 \\
\hline 180 & -0.007557 & 0.033442 & 0.0000002 \\
\hline 200 & -0.002445 & 0.013548 & 0.0000037 \\
\hline 300 & -0.000001 & 0.000013 & 0.0000022 \\
\hline
\end{tabular}

Na tabela VII temos intervalos de confiança aproximados de $95 \%$ para $R\left(y^{*}\right)$ considerando a "normalidade" assintótica de $\hat{R}\left(y^{*}\right)$ e $\hat{\phi}_{G J}(\lambda)$ para diferentes valores de $y^{*}=\ln \left(t_{0}\right)$. Encontramos diferentes intervalos de confiança para $R\left(y^{*}\right)$, especialmente para valores grandes de $t_{0}$, onde observamos intervalos mais precisos na parametrização $\phi_{G J}$, pois temos intervalos de valores positivos para $R\left(y^{*}\right)$. 
Tabela VII: Intervalo de $95 \%$ de confiança para $R\left(y^{*}\right)$ considerando as parametrizações $R\left(y^{*}\right)$ e $\phi_{G J}(\lambda)$

\begin{tabular}{|c|c|c|c|}
\hline$t_{0}$ & $\hat{R}\left(y^{*}\right)$ & Parametrização $R\left(y^{*}\right)$ & Parametrização $\phi_{G J}(\lambda)$ \\
\hline 5 & 0.99840 & $0.99790 ; 0.99889$ & $0.99796 ; 0.99889$ \\
\hline 20 & 0.96643 & $0.95619 ; 0.97665$ & $0.95622 ; 0.97665$ \\
\hline 60 & 0.67984 & $0.59853 ; 0.76114$ & $0.60071 ; 0.76189$ \\
\hline 80 & 0.48279 & $0.37384 ; 0.59174$ & $0.38227 ; 0.59804$ \\
\hline 120 & 0.16831 & $0.07536 ; 0.26125$ & $0.09608 ; 0.28662$ \\
\hline 180 & 0.01277 & $-0.00449 ; 0.03003$ & $0.00330 ; 0.04871$ \\
\hline 200 & 0.00408 & $-0.00287 ; 0.01103$ & $0.00074 ; 0.02226$ \\
\hline 300 & 0.000001 & $-0.000005 ; 0.000007$ & $0.00000 ; 0.00009$ \\
\hline
\end{tabular}

$\mathrm{Na}$ figura 6.9 temos os gráficos da função de verossimilhança "profile" para $\phi_{G J}(\lambda)$ (5.26) considerando os valores de $t_{0}$ e $\lambda$ dados na tabela VI. Comparando com a figura 6.8 observamos, em geral, uma melhoria na "normalidade" da função de verossimilhança "profile" na parametrização $\phi_{G J}(\lambda)$, especialmente para valores grandes de $t_{0}$.

Também podemos verificar a adequabilidadade da reparametrização proposta $\phi_{G J}(\lambda)$ usando o "t-plot" (ver capítulo 3) proposta por Hills e Smith (1993). (ver Figura 6.10 e 6.11). Na figura 6.11 observamos uma melhoria na linearidade do "t plots" considerando diferentes valores para $t_{0}$, o qual indica melhoramento na "normalidade" da função de verossimilhança $\phi_{G J}(\lambda)$. Na parametrização original, os "t-plots" têm forma curvilínea (ver figura 6.10) o qual indica não "normalidade" da função de verossimilhança para $R\left(y^{*}\right)$. 
$t_{0}=5$

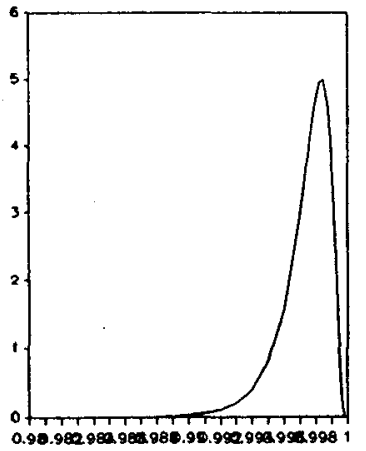

$t_{0}=120$

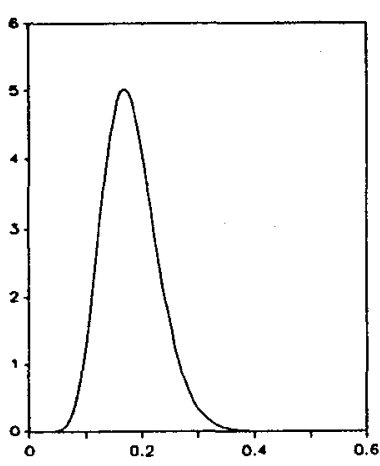

$t_{0}=20$

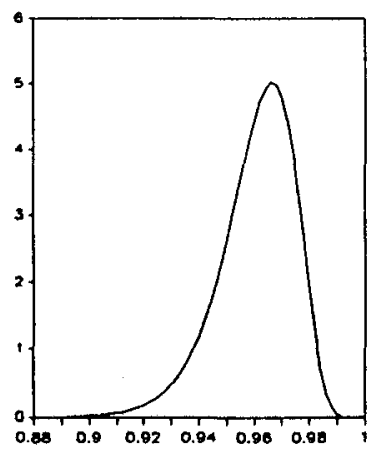

$t_{0}=180$

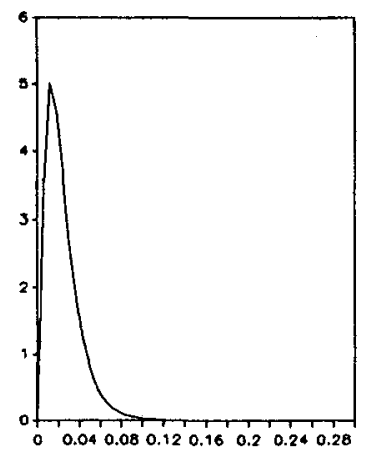

$t_{0}=60$

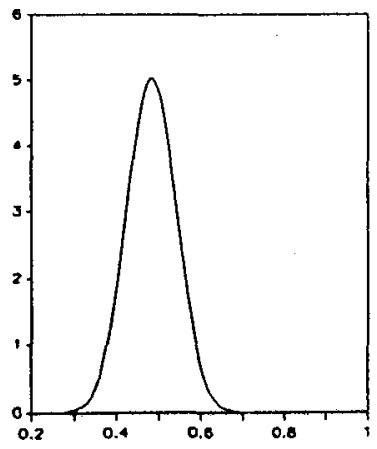

$t_{0}=200$

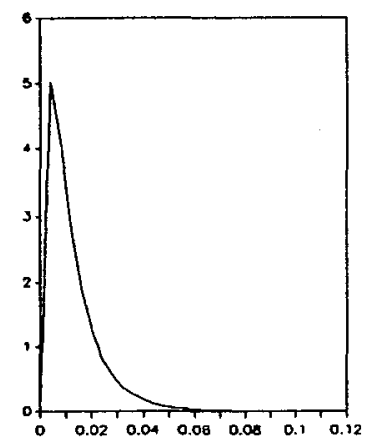

$t_{0}=80$

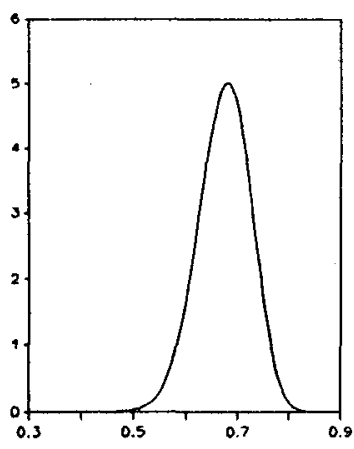

$t_{0}=300$

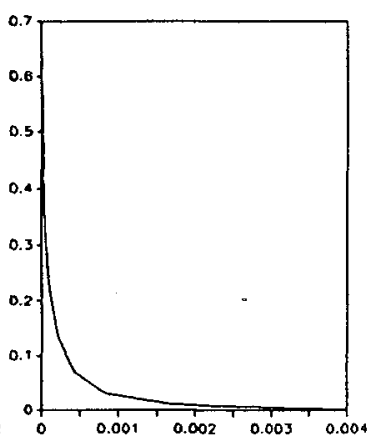

Figura 6.8: Função de Verossimilhança "Profile" Considerando a Parametrização $R\left(y^{*}\right)$

$t_{0}=5$

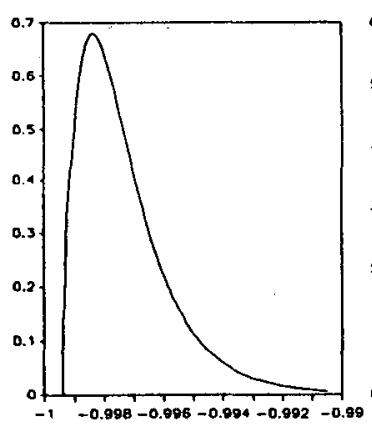

$t_{0}=120$ $t_{0}=20$

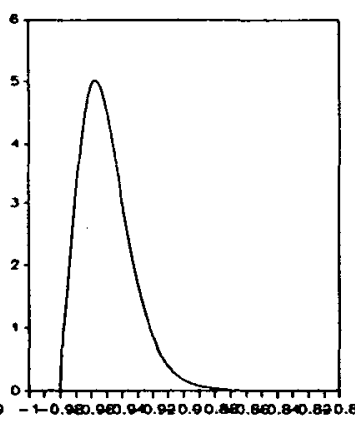

$t_{0}=180$
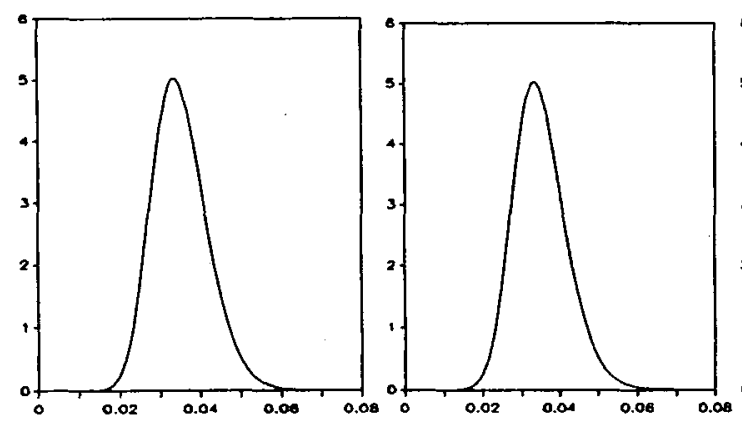

$t_{0}=200$
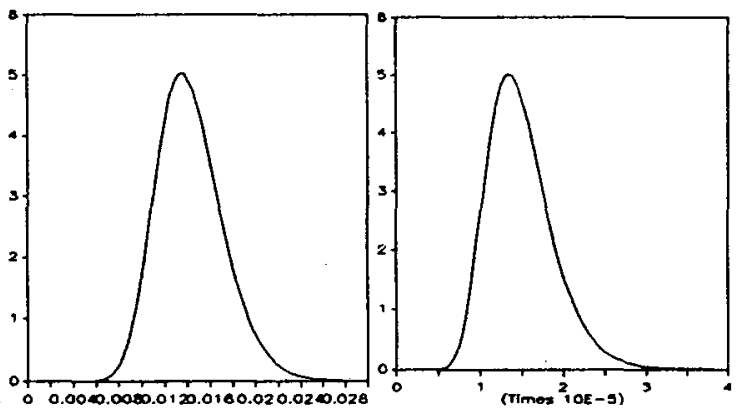

Figura 6.9: Função de Verossimilhança "Profile" Considerando a Parametrização $\phi_{G J}(\lambda)$ 
$t_{0}=5$

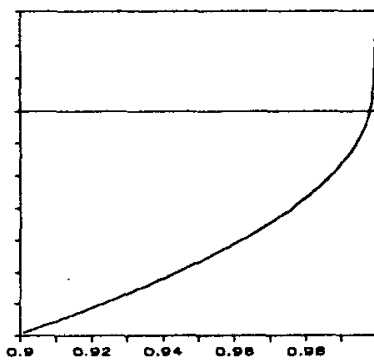

$t_{0}=120$

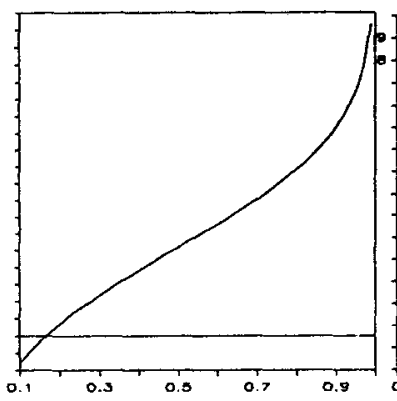

$t_{0}=20$

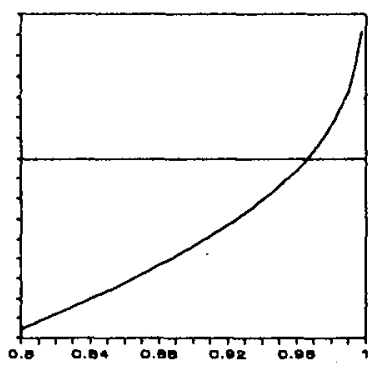

$t_{0}=180$

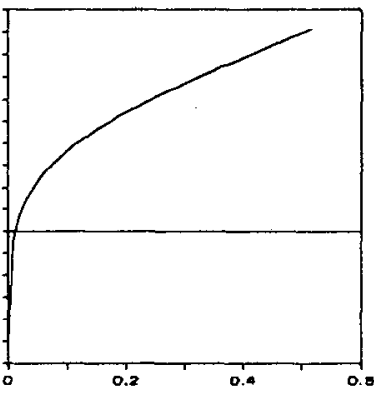

$t_{0}=60$

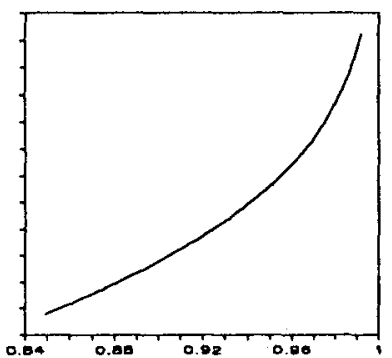

$t_{0}=200$

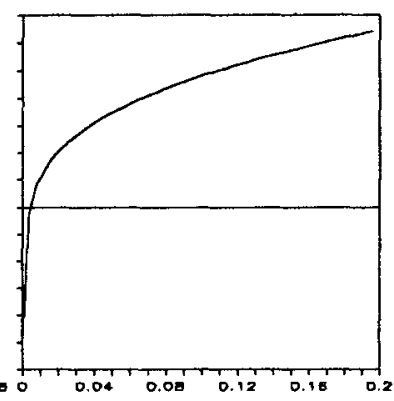

$t_{0}=80$

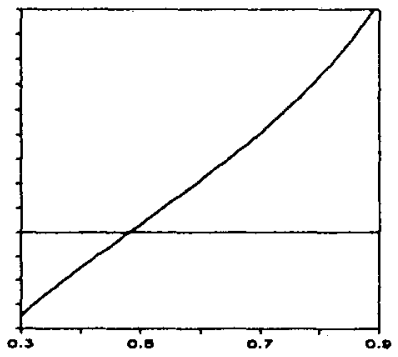

$t_{0}=300$

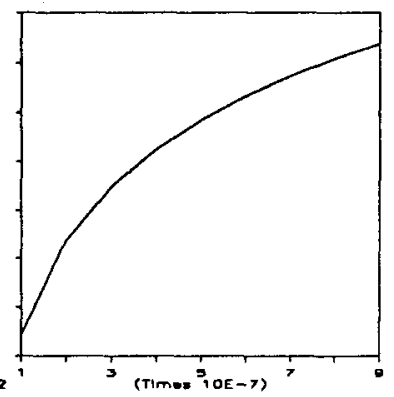

Figura 6.10: Gráficos de "t-plot" de Hills e Smith para a Função de Sobrevivência $R\left(y^{*}\right)$

$t_{0}=5$

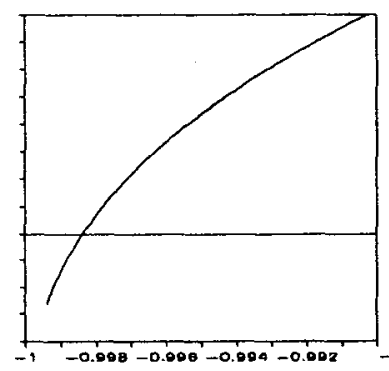

$t_{0}=120$

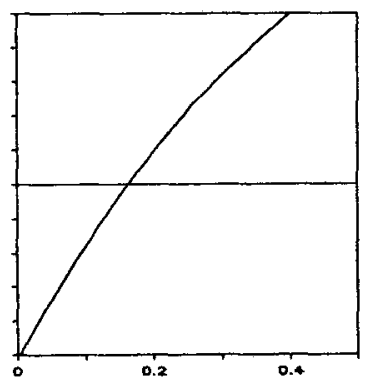

$t_{0}=20$

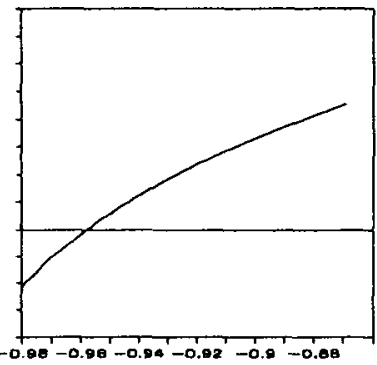

$t_{0}=180$

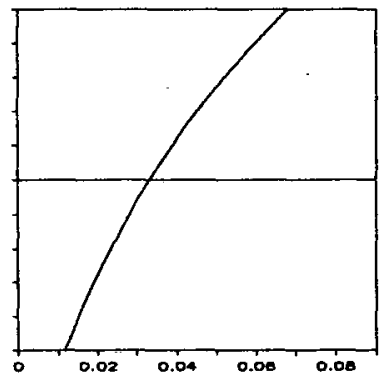

$t_{0}=60$

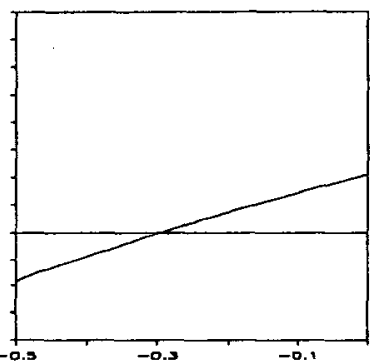

$t_{0}=200$

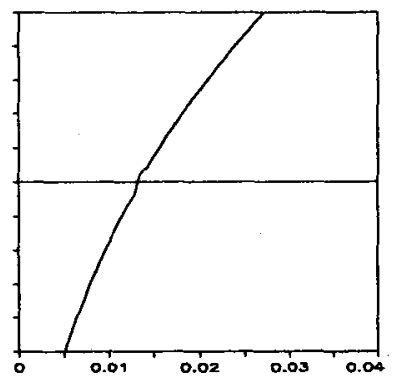

$t_{0}=80$

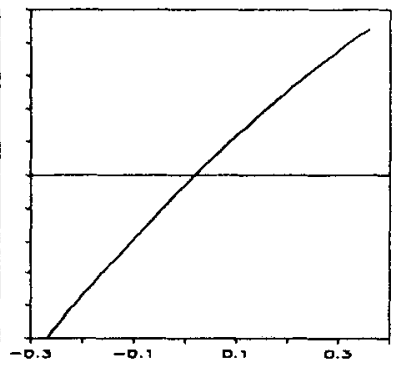

$t_{0}=300$

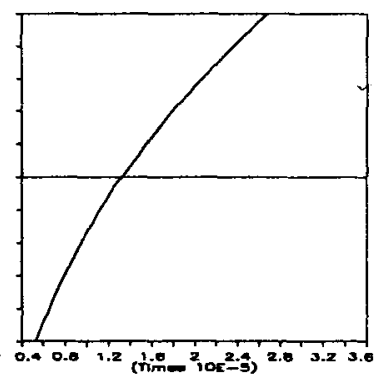

Figura 6.11: Gráficos de "t-plot" de Hills e Smith na Parametrização $\phi_{G J}(\lambda)$ 
Também podemos verificar a "normalidade" conjunta dos parâmetros de interesse como foi mencionado no capítulo 3. No caso de dois parâmetros geralmente verificamos a "normalidade" conjunta da função de verossimilhança com a ajuda de gráficos de contorno como foi feito no exemplo 1, com o modelo de Feigl e Zelen. Em situações com três ou mais parâmetros usamos a derivada terceira sumária (3.8).

$\mathrm{Na}$ tabela VIII temos a derivada terceira sumária para o logaritmo da função de verossimilhança $\ell\left(R, \beta_{0}, \sigma\right)$ e $\ell\left(\phi_{G J}, \beta_{0}, \sigma\right)$. Comparando os valores obtidos observamos que na parametrização $\phi_{G J}(\lambda)$ temos uma melhoria na "normalidade" conjunta da função de verossimilhança $L\left(\phi_{G J}, \beta_{0}, \sigma\right)$ para valores grandes de $t_{0}$.

Tabela VIII: Derivada Terceira Sumária $m^{2} \bar{B}^{2}$ para o logaritmo da função de verossimilhança $\ell\left(R, \beta_{0}, \sigma\right)$ e $\ell\left(\phi_{G J}, \beta_{0}, \sigma\right)$

\begin{tabular}{|c|c|c|}
\hline$t_{0}$ & $m^{2} \bar{B}^{2}\left(\hat{R}\left(y^{*}\right), \hat{\beta}_{0}, \hat{\sigma}\right)$ & $m^{2} \bar{B}^{2}\left(\hat{\phi}_{G J}(\lambda), \hat{\beta}_{0}, \hat{\sigma}\right)$ \\
\hline \hline 20 & 40.282 & 1.8407 \\
\hline 60 & 0.9014 & 0.5956 \\
\hline 80 & 1.5073 & 0.4796 \\
\hline 120 & 10.422 & 0.3830 \\
\hline 180 & 207.43 & 0.3885 \\
\hline 200 & 486.88 & 0.4172 \\
\hline
\end{tabular}

\subsubsection{Uma Análise Bayesiana}

A reparametrização proposta também pode ser muito útil na análise Bayesiana do modelo de regressão de valor extremo. Geralmente o estatístico considera o método de aproximação de integral ou um método numérico para obter a densidade a posteriori de interesse, e a precisão desses resultados usualmente depende da escolha de uma reparametrização apropriada. Como um caso especial, considere o uso do método de Laplace (ver Capítulo 2) para encontrar a densidade a posteriori marginal para $R\left(y^{*}\right)$ e $\phi_{G J}(\lambda)$. Considerando a priori não-informativa para $\beta_{0}, \beta_{1}$ e $\sigma$ baseado 
na regra multiparamétrica de Jeffreys (ver 5.27), temos nas figura 6.12 e 6.13 os gráficos das densidades a posteriori marginais para $R\left(y^{*}\right)$ e $\phi_{G J}(\lambda)$, respectivamente para os diferentes valores de $t_{0}$ (ver 5.31 e 5.34).

$\mathrm{Na}$ Figura 6.13 observamos uma melhoria significativa na normalidade das densidades a posteriori marginais $\pi\left(\phi_{G J} / d a d o s\right)$, especialmente para valores grandes de $t_{0}$ o que indica que a aproximação normal para a densidade a posteriori de $\phi_{G J}(\lambda)$ pode ser usada.

Considerando o uso do método de Laplace (ver Capítulo 2) na tabela IX temos momentos a posteriori aproximados para $R\left(y^{*}\right)$ considerando a densidade a posteriori conjunta para $\left(R\left(y^{*}\right), \beta_{0}, \sigma\right)$ e $\left(\phi_{G J}(\lambda), \beta_{0}, \sigma\right)$. Temos também na tabela IX os momentos a posteriori para $R\left(y^{*}\right)$ obtidos numericamente usando o método de Simpson. Comparando os valores obtidos pelo método de Laplace e os valores obtidos pela regra de Simpson, podemos ver claramente que a parametrização $\phi_{G J}(\lambda)$ melhora sensivelmente a precisão das estimativas, especialmente para valores grandes de $t_{0}$.

Tabela IX: Momentos a Posteriori para $R\left(y^{*}\right)$ para alguns valores de $t_{0}$

\begin{tabular}{|c||c|c|c|}
\hline \multirow{2}{*}{$t_{0}$} & \multicolumn{3}{|c|}{$E\left[R\left(y^{*}\right) /\right.$ dados $]$} \\
\cline { 2 - 4 } & Regra de Simpsom & $\begin{array}{c}\text { Laplace na Parametriza- } \\
\text { ção R }\end{array}$ & $\begin{array}{c}\text { Laplace na Parametri- } \\
\text { zação } \phi_{G J}\end{array}$ \\
\hline 5 & 0.998396 & 0.992666 & 0.996471 \\
\hline 20 & 0.966432 & 0.897550 & 0.963142 \\
\hline 60 & 0.681079 & 0.498351 & 0.675076 \\
\hline 80 & 0.485988 & 0.361186 & 0.482784 \\
\hline 120 & 0.174900 & 0.276818 & 0.176965 \\
\hline 150 & 0.059866 & 0.238719 & 0.063088 \\
\hline
\end{tabular}


$t_{0}=5$

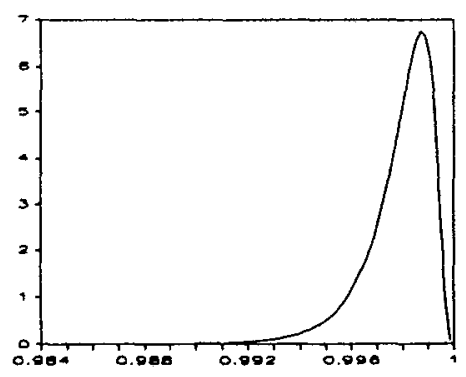

$t_{0}=120$

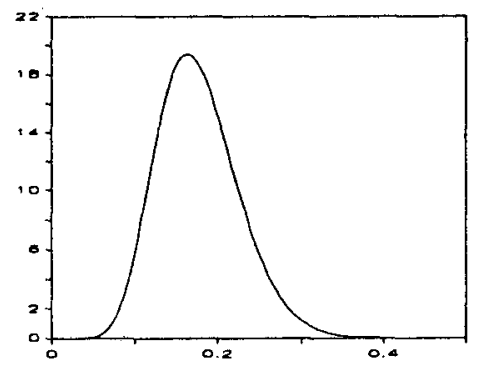

$t_{0}=20$

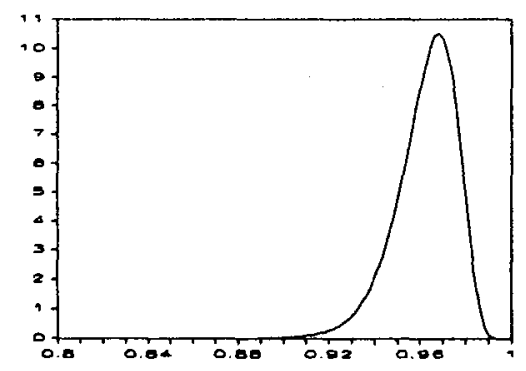

$t_{0}=180$

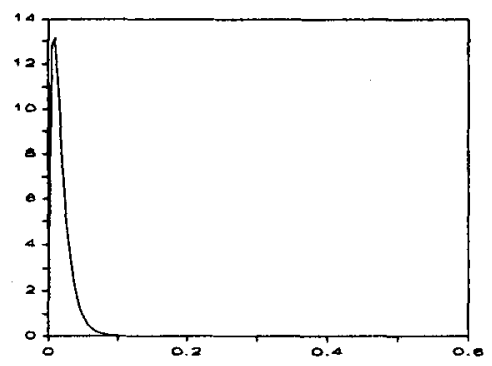

$t_{0}=60$

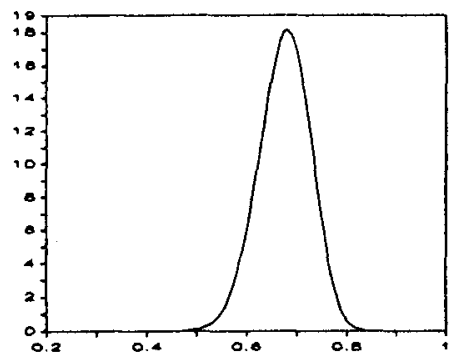

$t_{0}=200$

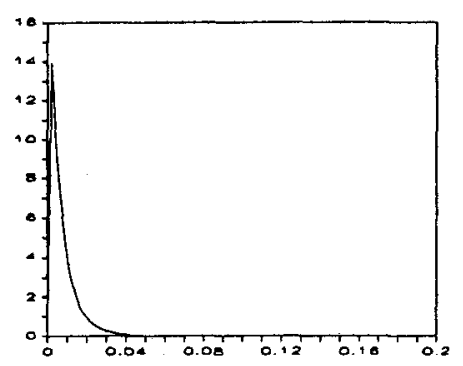

Figura 6.12: Densidade a Posteriori Marginal $\pi(R /$ dados $)$
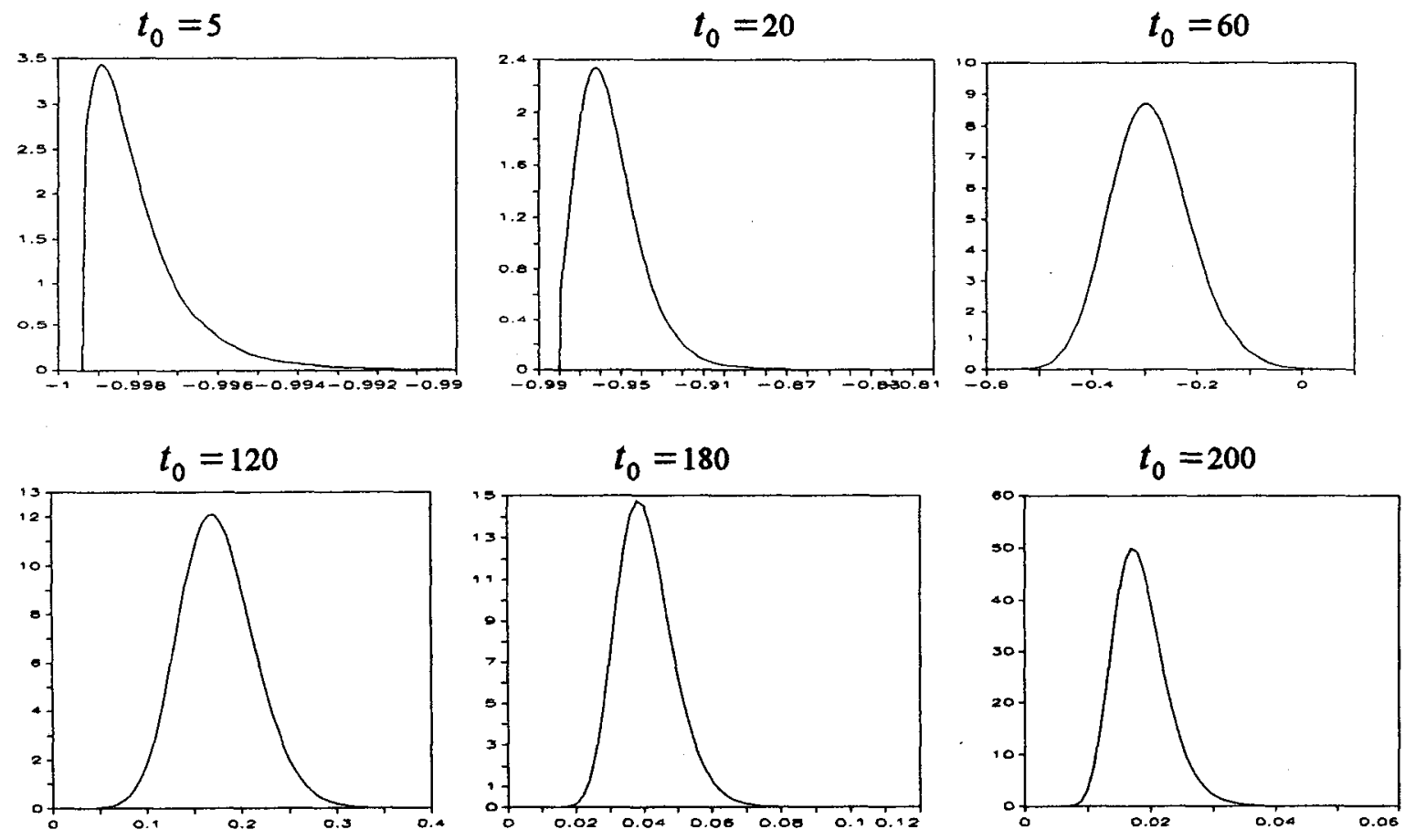

Figura 6.13: Densidade a Posteriori Marginal $\pi\left(\phi_{G J} /\right.$ dados $)$ 
Na tabela $\mathrm{X}$ temos os intervalos HPD aproximados $95 \%$ para $R\left(y^{*}\right)$ considerando a posteriori marginal $\pi(R /$ dados) $(5.31)$ (ver Apêndice A.2) e diferentes valores de $t_{0}$. Também temos na tabela $X$ intervalos HPD aproximados $95 \%$ para $R\left(y^{*}\right)$, considerando a posteriori marginal $\pi\left(\phi_{G J} /\right.$ dados $)$ (5.34). Observamos que os intervalos HPD aproximados $95 \%$ para $R\left(y^{*}\right)$ na parametrização $\phi_{G J}(\lambda)$ são muito próximos do intervalo de confiança $95 \%$ para $R\left(y^{*}\right)$, obtido via "normalidade" assintótica da função de verossimilhança na parametrização $\phi_{G J}(\lambda)$ dado na tabela VII. $\mathrm{O}$ que significa boa "normalidade" na parametrização $\phi_{G J}(\lambda)$.

Tabela X: Intervalos HPD aproximados $95 \%$ para $R\left(y^{*}\right)$ considerando as parametrizações $R\left(y^{*}\right)$ e $\phi_{G J}(\lambda)$

\begin{tabular}{|c|c|c|c|}
\hline$t_{0}$. & $R\left(y^{*}\right)$ & Parametrização $R\left(y^{*}\right)$ & Parametrização $\phi_{G J}(\lambda)$ \\
\hline 5 & 0.99840 & $0.99871 ; 100000$ & $0.99754 ; 0.99909$ \\
\hline 20 & 0.96643 & $0.95603 ; 0.97639$ & $0.95555 ; 0.97680$ \\
\hline 60 & 0.67984 & $0.59922 ; 0.76154$ & $0.59525 ; 0.77361$ \\
\hline 80 & 0.48279 & $0.37726 ; 0.59470$ & $0.39067 ; 0.60583$ \\
\hline 120 & 0.16831 & $0.08512 ; 0.27160$ & $0.10232 ; 0.45206$ \\
\hline 180 & 0.01277 & $0.00162 ; 0.03317$ & $0.00359 ; 0.08603$ \\
\hline 200 & 0.00408 & $0.00023 ; 0.01173$ & $0.00055 ; 0.06714$ \\
\hline
\end{tabular}

Em geral, o uso de reparametrizações na análise Bayesiana de modelos estatísticos tem certas implicações práticas, desde que possamos obter boa aproximação normal para a densidade a posteriori de interesse. Muitas vezes a utilização de métodos de aproximação para integrais (ver Capítulo 2) pode ter sua precisão comprometida se a densidade a posteriori de interesse não tem uma forma bem comportada 
(aproximadamente normal ) e a procura de uma parametrização adequada toma- se necessário.

Assim verificamos que a parametrização proposta por Guerrero e Johnson (1982) (ver 3.9), pode ser de grande interesse prático para melhorar a precisão das inferências assintóticas em funções de sobrevivência em um tempo especificado $t_{0}$ no modelo de regressão de valor extremo, considerando um valor apropriado para $\lambda$ obtido da derivada terceira padronizada do logaritmo da função de verossimilhança, localmente no estimador de máxima verossimilhança de $\phi_{G J}(\lambda)$. 


\section{CAPÍTULO 7}

\section{CONSIDERAÇÕES FINAIS E PERSPECTIVAS FU- TURAS}

Observamos que o uso de uma reparametrização apropriada para a função de sobrevivência num dado tempo pode melhorar de forma acentuada as inferências assintóticas, quer seja sob o enfoque clássico ou sob o enfoque Bayesiano. É importante salientar que usamos modelos de regressão com dados de sobrevivência assumindo as distribuições exponencial e de Weibull (valor extremo), mas os resultados desta dissertação poderiam ser generalizados para outros modelos de regressão considerando outras distribuições para os dados de sobrevivências.

Explorando a terceira derivada padronizada do logaritmo da função de verossimilhança apresentamos uma técnica muito simples para achar uma parametrização adequada para cada aplicação. Destacamos que os métodos alternativos existentes para achar uma parametrização adequada, em geral envolvem a solução de equações diferenciais, muitas vezes complicadas. Isso pode favorecer, em termos prático, uso da metodologia apresentada nesta dissertação por pesquisadores nas mais diversas áreas.

A seguir, destacamos alguns pontos importantes que poderão ser complementados no futuro:

1. Estudo das reparametrizações consideradas com outros modelos de regressão para dados de sobrevivências. 
2. Consideração de outras distribuições de probabilidade para os tempos de sobrevivências.

3. Estudo detalhado dos efeitos das reparametrizações consideradas assumindo dados censurados.

4. Aplicações em modelos usados em testes de sobrevivência acelerados ( ver por exemplo, Mann, Schafer e Singpurawalla, 1974; Nelson, 1970, 1971; ou Achcar e Louzada Neto, 1992).

5. Uso de outras densidades a priori para verificar seu efeito nas inferências obtidas com o modelo reparametrizado. Poderia ser considerado, além da densidade a priori não-informativa de Jeffreys utilizada neste trabalho, outras prioris, como a densidade a priori de Zellner ou a densidade a priori conjugada, pois em aplicações industriais, é muito comum o uso de densidades a priori informativas, pois os engenheiros podem ter informações sobre o mecanismo da distribuição dos parâmetros de interesse.

6. Considerar outras reparametrizações para funções de sobrevivências (ver por exemplo, Aranda-Ordaz, 1981).

7. Para achar os momentos a posteriori poderia ser considerado além da função de perda quadrática estudada, a função de perda assimétrica, nas diferentes reparametrizações.

8. Desenvolver Software para as técnicas desenvolvidas: é importante destacar que vários programas individuais foram desenvolvidos neste trabalho (ver Apêndice A4). 


\section{APÊNDICE}

\section{A.1) Derivada do Logaritmo da Função de Verossimilhança "Profile" para $\phi_{G J}(\lambda)$ - Caso Exponencial (Modelo de Feigl e Zelen)}

O logaritmo da função de verossimilhança "profile" para $\phi_{G J}=\phi_{G J}(\lambda)$ é dado por:

$$
\ell_{\hat{\phi}_{2}}\left(\phi_{G J}\right)=-n \ln t_{0}+n \ln B\left(\phi_{G J}\right)+n \hat{\phi}_{2}\left(x_{0}-\bar{x}\right)-\frac{B\left(\phi_{G J}\right) A^{*}\left(\hat{\phi}_{2}\right)}{t_{0}},
$$

onde $B\left(\phi_{G J}\right)=\ln \left[1+A\left(\phi_{G J}\right)\right], A\left(\phi_{G J}\right)=\left(\phi_{G J}+1\right)^{-1 / \lambda}$ e $A^{*}\left(\hat{\phi}_{2}\right)=\sum_{i=1}^{n} t_{j} e^{-\hat{\phi}_{2}\left(x_{i}-x_{0}\right)}$.

As três primeiras derivadas de $\ell_{\hat{\phi}_{2}}\left(\phi_{G j}\right)$ são dadas por:

$$
\begin{aligned}
& \ell_{\hat{\phi}_{2}}^{\prime}\left(\phi_{G J}\right)=n \frac{B^{\prime}\left(\phi_{G J}\right)}{B\left(\phi_{G J}\right)}-\frac{B^{\prime}\left(\phi_{G J}\right)}{t_{0}} A^{*}\left(\hat{\phi}_{2}\right), \\
& \ell_{\hat{\phi}_{2}}^{\prime \prime}\left(\phi_{G J}\right)=n \frac{B^{\prime \prime}\left(\phi_{G J}\right)}{B\left(\phi_{G J}\right)}-n\left(\frac{B^{\prime}\left(\phi_{G J}\right)}{B\left(\phi_{G J}\right)}\right)^{2}-\frac{B^{\prime \prime}\left(\phi_{G J}\right) A^{*}\left(\hat{\phi}_{2}\right)}{t_{0}}
\end{aligned}
$$

e

$$
\ell_{\phi_{2}}^{\prime \prime \prime}\left(\phi_{G J}\right)=n\left(\frac{B^{\prime \prime \prime}\left(\phi_{G J}\right)}{B\left(\phi_{G J}\right)}\right)-3 n \frac{B^{\prime}\left(\phi_{G J}\right) B^{\prime \prime}\left(\phi_{G J}\right)}{\left[B\left(\phi_{G J}\right)\right]^{2}}-\frac{B^{\prime \prime \prime}\left(\phi_{G J}\right) A^{*}\left(\hat{\phi}_{2}\right)}{t_{0}}
$$


onde ,

$$
\begin{aligned}
& B^{\prime}\left(\phi_{G J}\right)=\frac{A^{\prime}\left(\phi_{G J}\right)}{1+A\left(\phi_{G J}\right)}, \\
& B^{\prime \prime}\left(\phi_{G J}\right)=\frac{A^{\prime \prime}\left(\phi_{G J}\right)}{1+A\left(\phi_{G J}\right)}-\left(\frac{A^{\prime}\left(\phi_{G J}\right)}{1+A\left(\phi_{G J}\right)}\right)^{2}, \\
& B^{\prime \prime \prime}\left(\phi_{G J}\right)=\frac{A^{\prime \prime \prime}\left(\phi_{G J}\right)}{1+A\left(\phi_{G J}\right)}-\frac{3 A^{\prime}\left(\phi_{G J}\right) A^{\prime \prime}\left(\phi_{G J}\right)}{\left(1+A\left(\phi_{G J}\right)\right)^{2}}+2\left(\frac{A^{\prime}\left(\phi_{G J}\right)}{1+A\left(\phi_{G J}\right)}\right)^{3}, \\
& A^{\prime}\left(\phi_{G J}\right)=-\frac{1}{\lambda}\left(\phi_{G J}+1\right)^{-\frac{1}{\lambda}-1}, \\
& A^{\prime \prime}\left(\phi_{G J}\right)=\frac{1}{\lambda}(1 / \lambda+1)\left(\phi_{G J}+1\right)^{-\frac{1}{\lambda}-2}
\end{aligned}
$$

e

$$
A^{\prime \prime \prime}\left(\phi_{G J}\right)=-\frac{1}{\lambda}(1 / \lambda+1)(1 / \lambda+2)\left(\phi_{G J}+1\right)^{-\frac{1}{\lambda}-3} .
$$

Também,

$$
A^{*}\left(\phi_{2}\right)=\sum_{i=1}^{n} t_{i}\left(x_{i}-x_{0}\right) e^{-\left(x_{i}-x_{0}\right) \phi_{2}}
$$

e

$$
A^{* \prime}\left(\phi_{2}\right)=\sum_{i=1}^{n} t_{i}\left(x_{i}-x_{0}\right)^{2} e^{-\left(x_{i}-x_{0}\right) \phi_{2}} .
$$




\section{A.2) Obtenção de Intervalo HPD}

Em inferência Bayesiana, em geral temos interesse em determinar uma região $R$ ( do espaço paramétrico) para o qual a probabilidade de conter a densidade a posteriori para um determinado parâmetro $\theta$ é $\left(1-\alpha^{\prime}\right)$.

Como discutido em Box e Tiao (1973), tal região é chamada de região "a posteriori de maior probabilidade" (HPD) $100\left(1-\alpha^{\prime}\right) \%$ se,

$$
\int_{R} \pi(\theta \mid d a d o s) d \theta=1-\alpha^{\prime}
$$

onde $\pi(\theta \mid$ dados $)$ é a densidade a posteriori para $\theta$, e

$$
\pi\left(\theta_{1} \mid \text { dados }\right) \geq \pi\left(\theta_{2} \mid \text { dados }\right) \text { para todo } \theta_{1} \in R \text { e } \theta_{2} \notin R
$$

(ver por exemplo, Martz e Waller, 1982).

Podemos definir a "densidade a posteriori relativa para $\theta$ " dada por:

$$
R=R(\theta \mid \text { dados })=\frac{\pi(\theta \mid \text { dados })}{\pi(\tilde{\theta} \mid \text { dados })},
$$

onde $\pi(\theta \mid d a d o s)$ é definida em (A3.1) e $\pi(\tilde{\theta} \mid$ dados $)$ é o valor da densidade a posteriori para $\theta$ calculado em $\tilde{\theta}$ ( $\tilde{\theta}$ é a moda a posteriori para $\theta$ ), e o conjunto de valores de $\theta$ tais que $R \geq \theta^{\prime}$ é chamado de intervalo de HPD para $\theta$. 
Considerando a aproximação $\chi^{2}$, isto é $-2 \log R \sim \chi_{(1)}^{2}$,

$-\alpha^{\prime} \approx P\left(\chi_{(1)}^{2} \leq-2 \log \theta^{\prime}\right)$

Portanto se $\theta^{\prime}=0.147, P\left(\chi_{(1)}^{2} \leq-2(-1.92)\right) \approx 0.95$, e um intervalo HPD aproximado $95 \%$ para $\theta$ pode ser obtido graficamente construindo-se o gráfico de $\theta$ versus $R$ e traçando-se uma reta em $R=0.147$.

A metodologia descrita acima pode ser encontrado em LOUZADA, N. F. (1991). (Dissertação de Mestrado).

\section{A.3) LISTAGEM DE PROGRAMAS DE COMPUTADOR}

\section{A.3.1) PROgRaMA PARA CALCULAR A DENSIDAde A POSTERIORI MARGINAL PARA O PARÂMETRO $\phi_{G J}$ DO MODELO DE FEIGL E ZELEN USANDO O MÉTOdO DE LAPLACE ( PROGRAMADO NO MINITAB)}

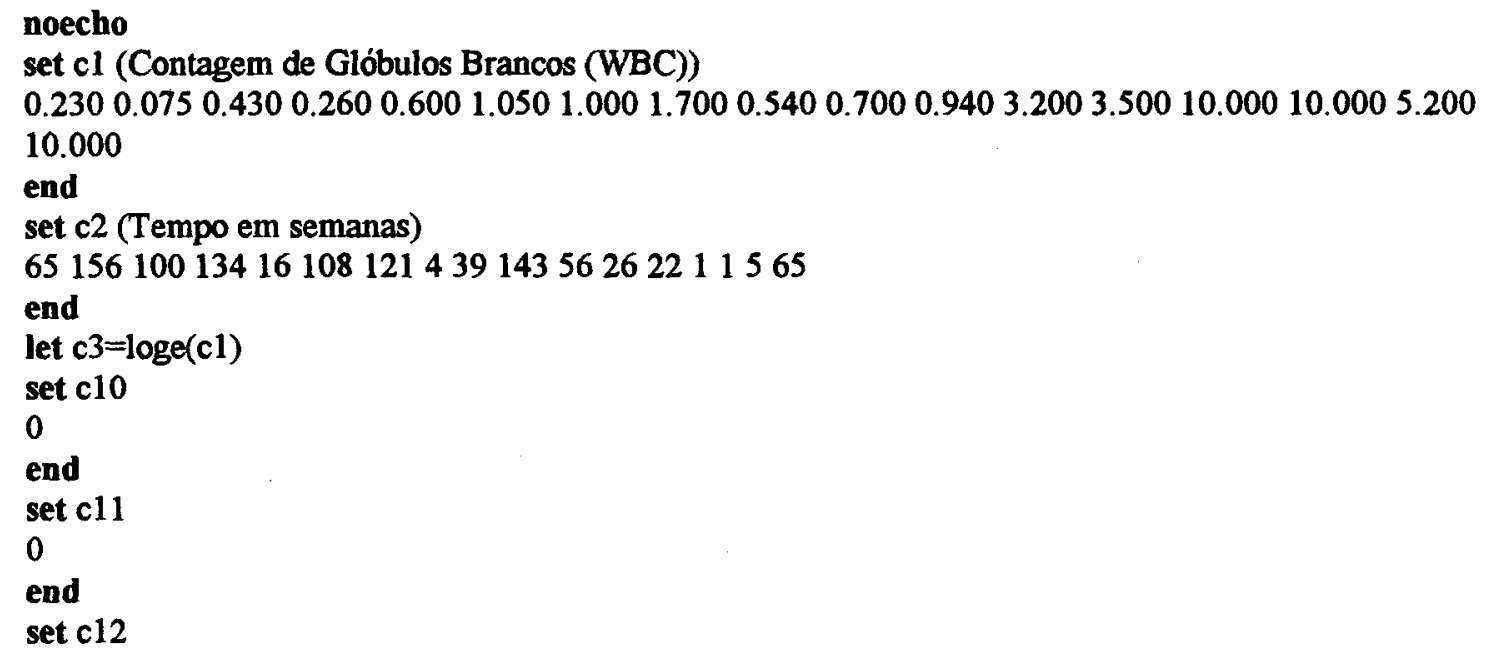




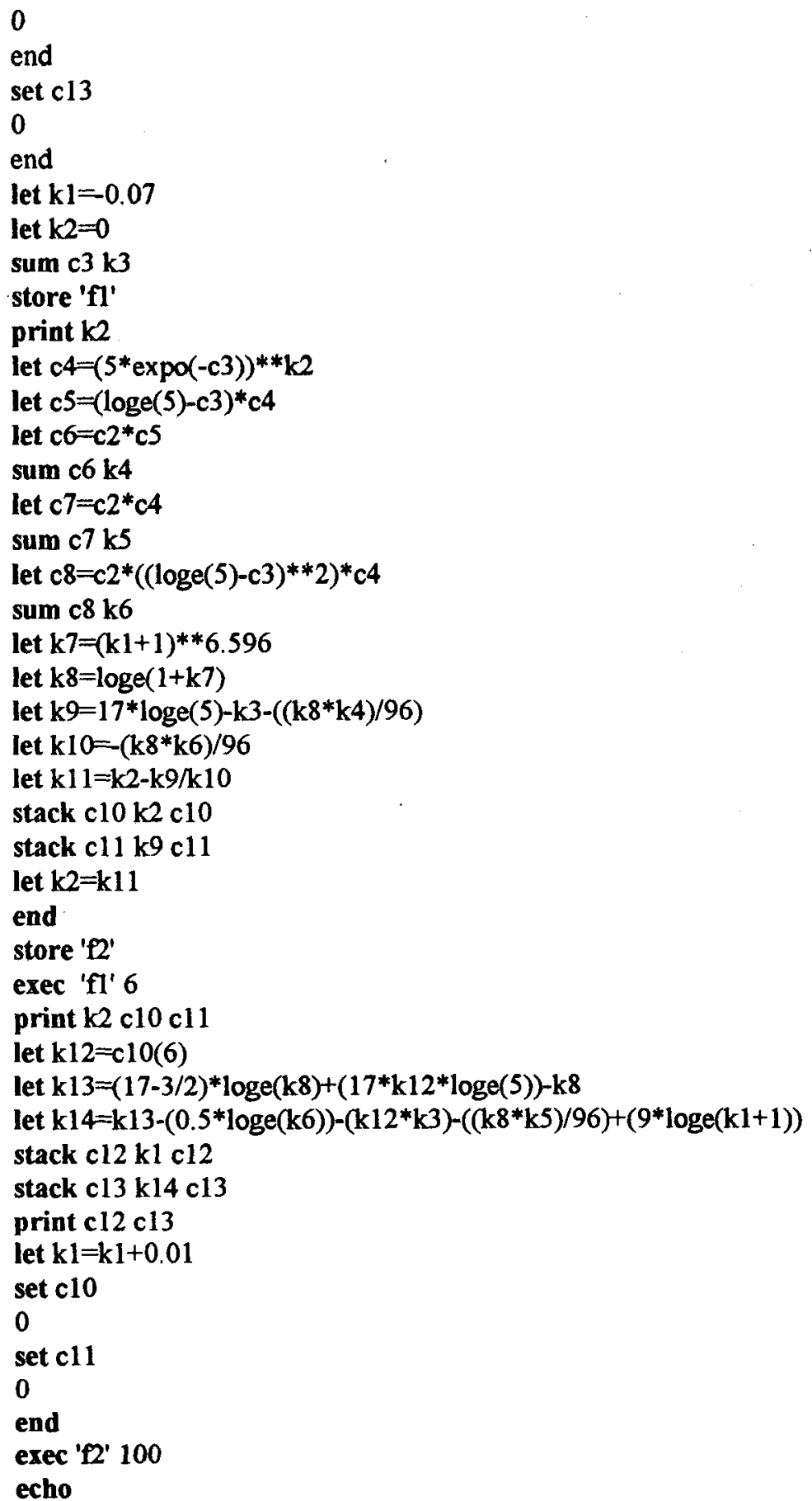




\section{A.3.2) PROGRAMA PARA CALCULAR A DENSIDADE A POSTERIORI MARGINAL PARA O PARÂMETRO $\phi_{G J}$ DO MODELO DE FEIGL E ZELEN USANDO O MÉTODO DE QUADRATURA GAUSSIANA (PROGRAMADO NO MINITAB)}

noecho

set cl (Contagem de Glóbulos Branco)

0.2300 .0750 .4300 .2600 .6001 .0501 .0001 .7000 .5400 .7000 .9403 .2003 .50010 .00010 .0005 .200 10.000

end

set $\mathrm{c} 2$ (tempo em semanas)

651561001341610812143914356262211565

end

let $c 3=\log (\mathrm{c} 1)$

read c5 c6 (dados da tabelal capítulo 2)

$0.3142404 \quad 0.5701352$

$\begin{array}{ll}-0.3142404 & 0.5701352\end{array}$

$\begin{array}{ll}0.9477884 & 0.02604923\end{array}$

$\begin{array}{cc}-0.9477884 & 0.02604923\end{array}$

$\begin{array}{ll}1.5976826 & 0.0516080\end{array}$

$\begin{array}{ll}-1.5976826 & 0.0516080\end{array}$

$\begin{array}{ll}2.2795071 & 0.0039054\end{array}$

$\begin{array}{ll}-2.2795071 & 0.003054\end{array}$

$\begin{array}{lll}3.0206370 & 0.0000857\end{array}$

$\begin{array}{ll}-3.0206370 & 0.0000857\end{array}$

$\begin{array}{lll}3.8897249 & 0.0000003\end{array}$

$\begin{array}{ll}-3.8897249 & 0.0000003\end{array}$

end

let $\mathbf{k} \mathbf{l}=0.07$

let $\mathrm{k} 5=1$

sum c3 k3

store ' $\mathrm{hl}$ '

let $\mathrm{k} 2=\mathrm{c} 5(\mathrm{k} 5)$

let $\mathrm{c} 4=\mathrm{c} 2 *(5 * \operatorname{expo}(-\mathrm{c} 3))^{* * \mathrm{k} 2}$

sum $\mathrm{c} 4 \mathrm{k} 4$

let $\mathrm{k} 6=(\mathrm{k} 1+1)$

let $\mathrm{k} 7=(\mathrm{k} 6 * * 6.596)$

let $k 8=(1+k 7)$

let $\mathrm{k} 9=\operatorname{loge}(\mathrm{k} 8)$

let $k 10=(16 * \log e(k 9))+(9 * \log e(k 6))-k 9+(17 * k 2 * \log e(5))$

let $\mathrm{k} 10=\mathrm{k} 10-(\mathrm{k} 2 * \mathrm{k} 3)-(\mathrm{k} 9 * \mathrm{k} 4) / 96$

let $\mathrm{k} 11=\operatorname{expo}(\mathrm{k} 10)$

let $\mathrm{c} 7(\mathrm{k} 5)=\mathrm{k} 11$

let $\mathrm{k} 5=\mathrm{k} 5+1$

end

store ' $\mathrm{h} 2$ '

exec ' $h l$ ' 12

let $c 8=c 6 * c 7 * \operatorname{expo}(c 5 * * 2)$

sum $\mathrm{c} 8 \mathrm{k12}$

stack $c 9$ k1 c9

stack $\mathrm{c} 10 \mathrm{k} 12$ c10

print k1 c5-c7 c9 c10 c13

let $\mathrm{kl}=\mathrm{kl}+0.01$

set $c 7$ 
0

let $k S=1$

end

exec 'h2' 100

echo

\section{A.3.3)PROGRAMAPARA PARA ENCONTRAR A CONSTANTE PADRONIZADORA PARA A VEROSSIMULHANÇA PADRONIZADA NA PARAMETRIZAÇÃo R (PROGRAMAdo NO TURBO PASCAL)}

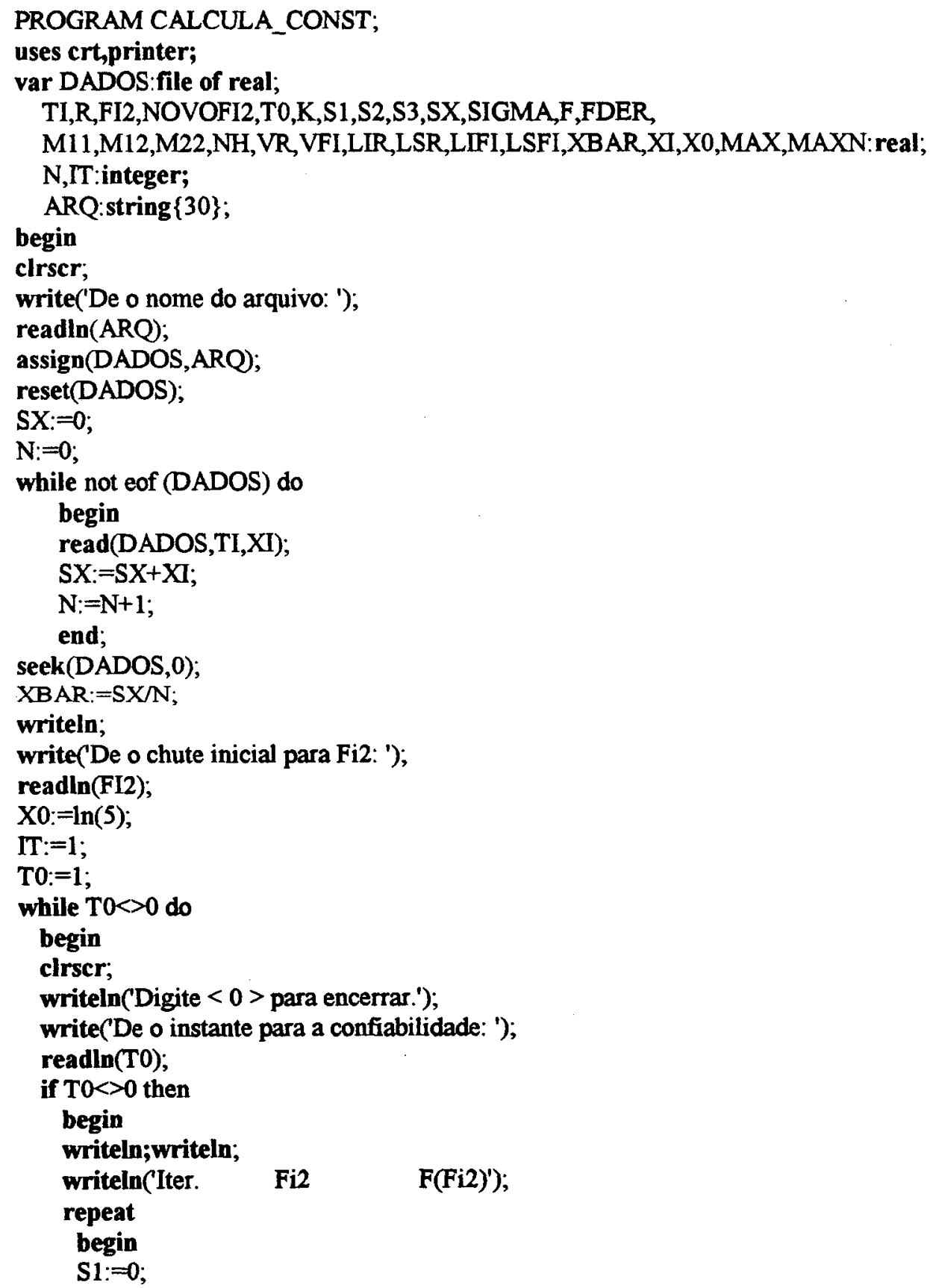




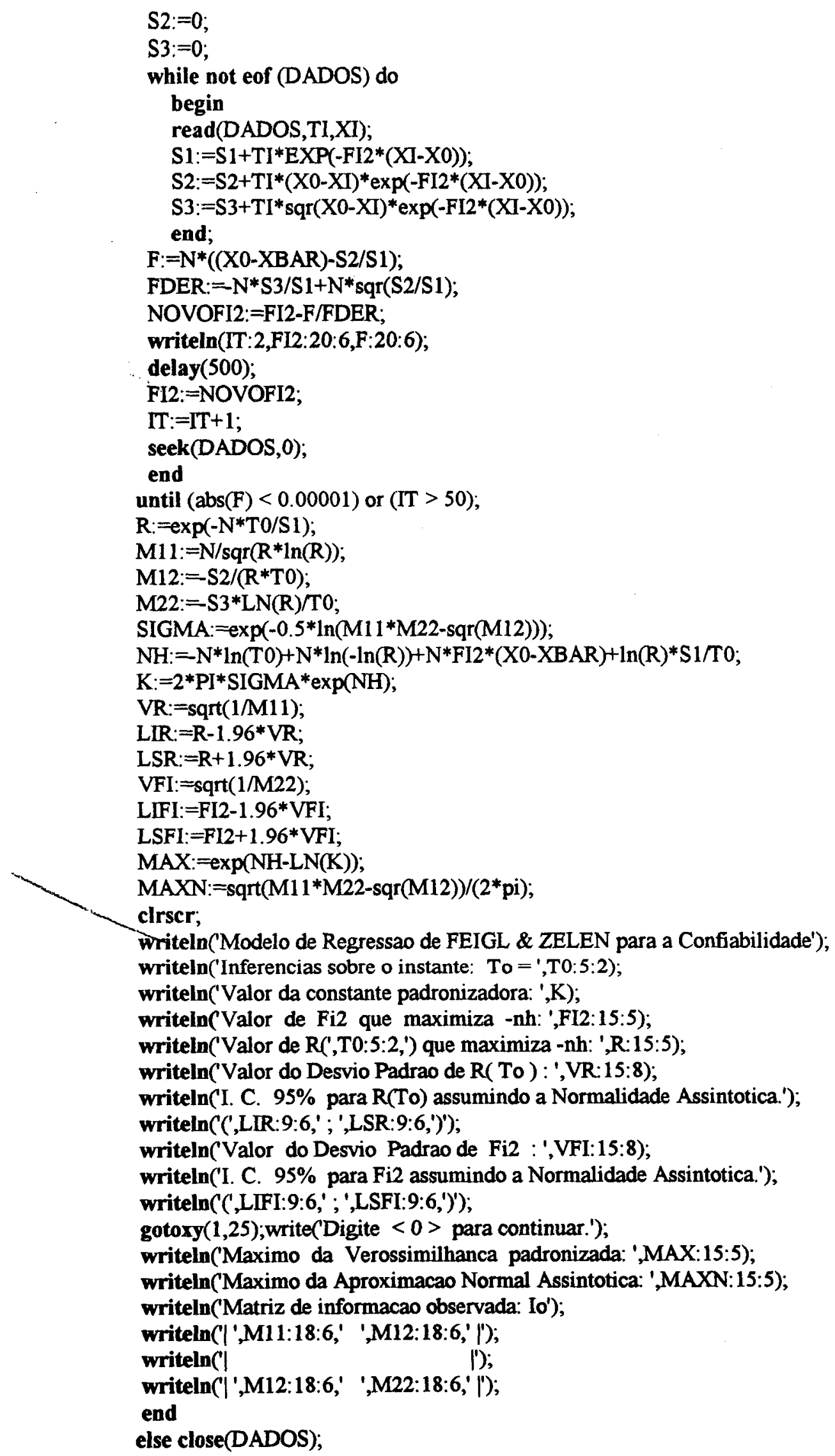


end;

clrscr;

end.

\section{A.3.4) PROGRAMA PARA ENCONTRAR O CONTORNO DA VEROSSIMILH PADRONIZADA NA PARAMETRIZAÇÃO R E $\phi_{2}$ (PROGRAMADO NO TURBO PASCAL)}

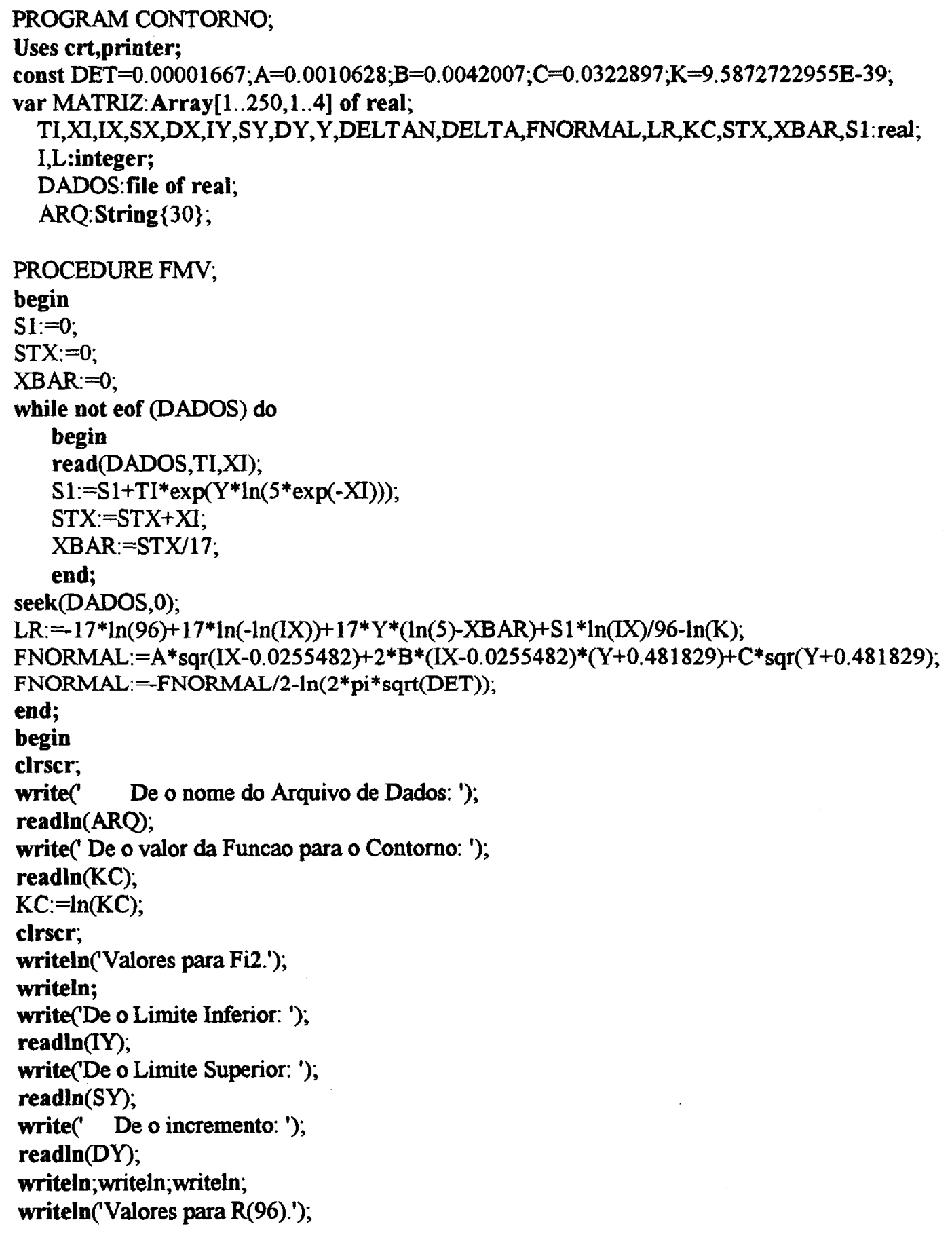




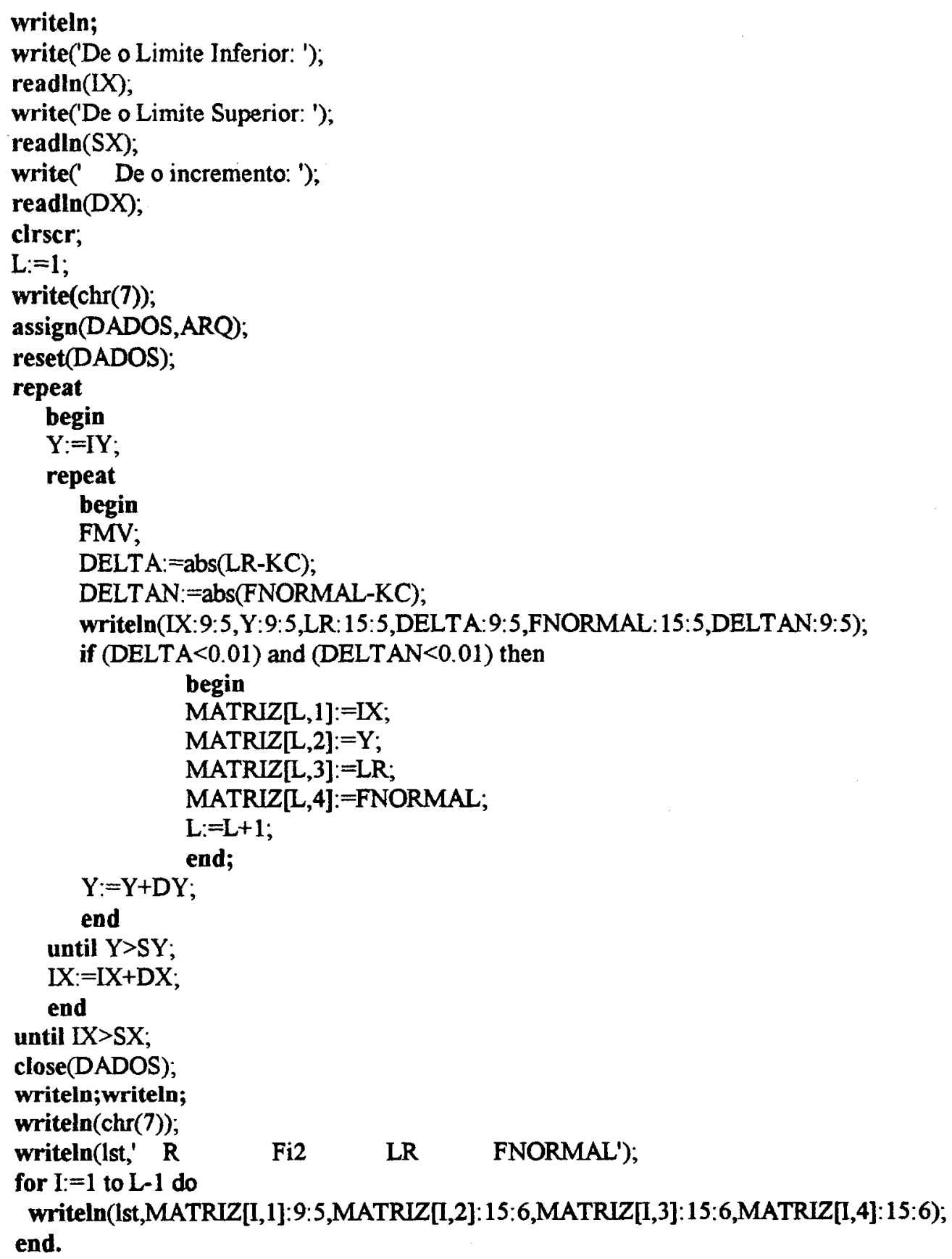




\section{A.3.5) PROGRAMA PARA ENCONTAR A DERIVADA TERCEIRA SUMÁRIA (KASS E SLATE 1992) PARA DOIS PARÂMETROS}

noecho

set $\mathrm{cl}$

32(1 2)

end

set c2

$16\left(\begin{array}{llll}1 & 1 & 2 & 2\end{array}\right)$

end

set $c 3$

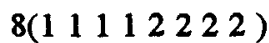

end

set c4

$8(1) 8(2) 8(1) 8(2) 8(1) 8(2) 8(1) 8(2)$

end

set $c 5$

16(1) 16(2) 16(1) 16(2)

end

set $\mathrm{c} 6$

32(1) 32(2)

end

let $\mathrm{c} 7=\mathrm{c} 1{ }^{*} \mathrm{c} 2$

let $c 8=c 3^{*} c 4$

let $c 9=c 5 * c 6$

let $\mathrm{c} 10=\mathrm{cl}^{*} \mathrm{c} 2 * \mathrm{c5}$

let $\mathrm{cl} 1=\mathrm{c} 3 * \mathrm{c} 4 * \mathrm{c} 6$

let $\mathrm{kl}=0.000629$

let $\mathrm{k} 2=0.000144$

let $\mathrm{k} 3=0.000339$

code (1) k1 c7 c20

code (2) k2 c20 c20

code (4) k3 c20 c20

code (1) k1 c8 c21

code (2) k2 c21 c21

code (4) k3 c21 c21

code (1) k1 c9 c22

code (2) k2 c22 c22

code (4) k3 c22 c22

print c20 c21 c22

let $\mathrm{k} 4=16519.73$

let $\mathrm{k} 5=40044.39$

let $k 6=42130.92$

let $\mathrm{k} 7=161667.91$

code (1) k4 c10 c23

code (2) k5 c23 c23

code (4) k6 c23 c23

code (8) k7 c23 c23

code (1) k4 c11 c24

code (2) k5 c24 c24

code (4) k6 c24 c24

code (8) k7 c24 c24

print c23 c24

let $\mathrm{c} 12=\mathrm{c} 20^{*} \mathrm{c} 21 * \mathrm{c} 22 * \mathrm{c} 23 * \mathrm{c} 24$

sum c12 k8 
print $k 8$

\section{A.3.6) PROGRAMA PARA ENCONTAR A DERTVADA TERCEIRA SUMÁRIA (KASS E SLATE 1992) PARA TRÊS PARÂMETROS}

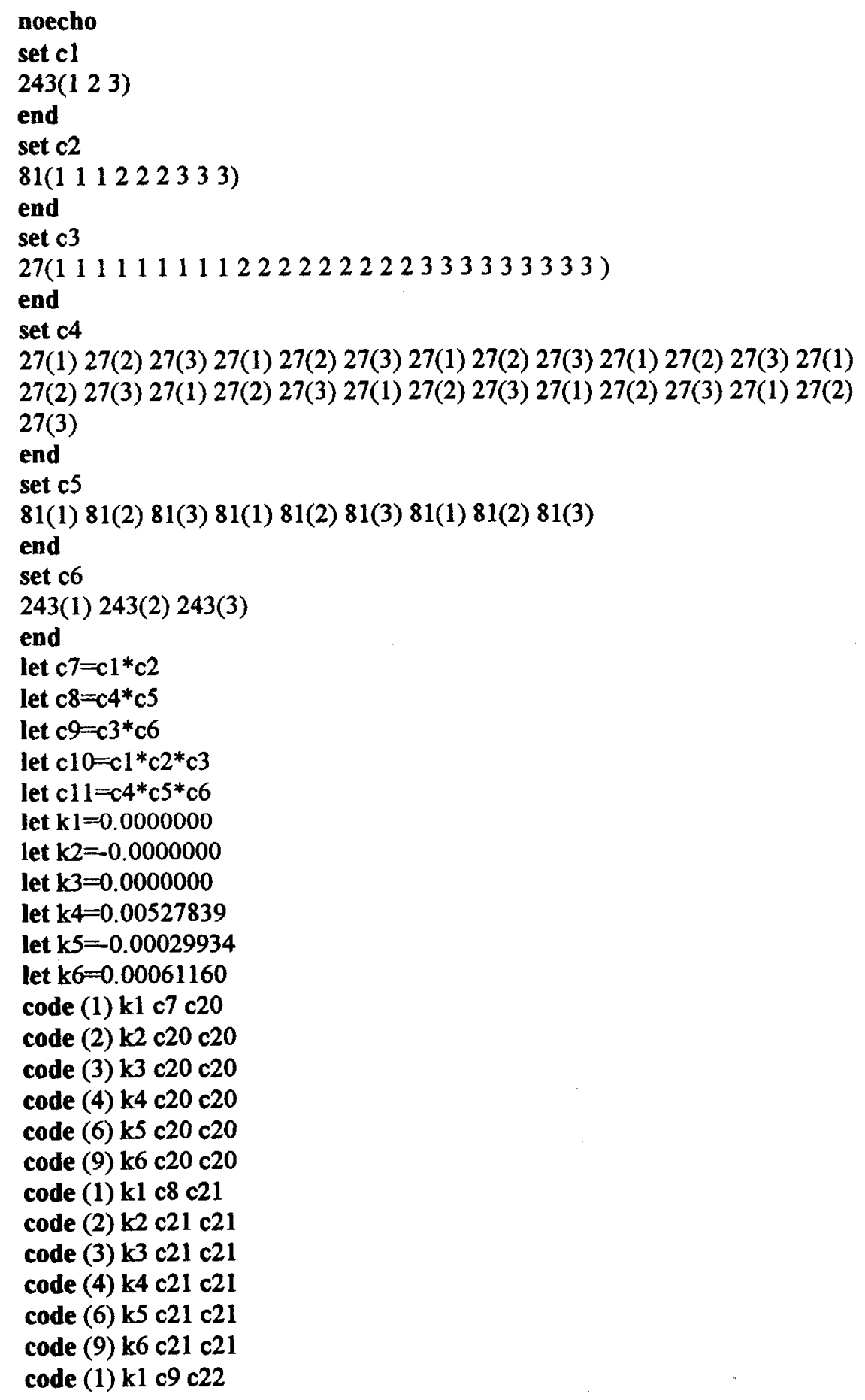


code (2) $k 2$ c22 c22

code (3) k3 c22 c22

code (4) k4 c22 c22

code (6) k5 c22 c22

code (9) k6 c22 c22

let $\mathrm{k} 7=1.239090 \mathrm{E}+17$

let $\mathrm{k} 8=0$

let $\mathrm{k}=14965884$

let $\mathrm{k} 10=0$

let $\mathrm{k} 11=0$

let $\mathrm{k} 12=31.9407$

let $k 13=16665473$

let $\mathrm{k} 14=1070.67$

let $k 15=1222.08$

let $k 16=13998.1$

code (1) k7 c10 c23

code (2) k8 c23 c23

code (3) k9 c23 c23

code (4) k10 c23 c23

code (6) k11 c23 c23

code (8) k12 c23 c23

code (9) k13 c23 c23

code (12) k14 c23 c23

code (18) k15 c23 c23

code (27) k16 c23 c23

code (1) k7 c11 c24

code (2) k8 c24 c24

code (3) k9 c24 c24

code (4) k10 c24 c24

code (6) k11 c24 c24

code (8) k12 c24 c24

code (9) k13 c24 c24

code (12) k14 c24 c24

code (18) k15 c24 c24

code (27) k16 c24 c24

let $\mathrm{c} 12=\mathrm{c} 20 * \mathrm{c} 21{ }^{*} \mathrm{c} 22 * \mathrm{c} 23 * \mathrm{c} 24$

sum $\mathrm{cl} 2 \mathrm{k17}$

print k17

echo 


\section{REFERÊNCIAS}

ABRAMOWITZ, M. ; STEGUN, I (1965). Handbook of Mathematical Functions, Dover.

ACHCAR, J. A. (1985). Modelos de Regressão Com Dados Censurados. São Paulo, Relatório Técnico do IME-USP, 18p.

ACHCAR, J. A. ; BROOKMEYER, R. ; HUNTER, W. G. (1985). An Application of Bayesian Analisis to Medical Follow-Up Data. Statistics in Medicine, vol 4, 509-520.

ACHCAR, J. A. ; BOLFARINE, H. (1986). Use of Accurate Aproximations for Poterior Densities in Regression Models With Censored Data. Rev. da Sociedade Chilena de Estadistica, 3(2), 84-104.

ACHCAR, J. A.; DINIZ, C. A. R. (1986). Comparação de dois Tratamentos com Dados de Sobrevivência Censurados e Transformados para uma Distribuição de Valor Extremo.Revista Mat. Estatística, 4/5: 45-55.

ACHCAR, J. A. ; BOLFARINE, H. (1988). Preditive Densities in Survival Analysis With Generalized Gamma Regression Model. Revista Brasileira de Probabilidade e Estatistica. 2, pp. 23-31. 
ACHCAR, J. A; SMITH, A. F. M. (1990). Aspects of Reparametrization in Aproximate Bayesian Inference. Bayesian and Likelihood Methods in Statistics and Econometrics. Nort-Holland, Elsevier Science Publishers B. V., Amsterdam, North - Holland.

ACHCAR, J. A. (1990). Reparametrization and Acuracy of Laplace. Approximations for Posterior Moments. Revista de Matemática e Estatística, 8, 23-30.

ACHCAR, J. A. (1991). An Useful Reparametrization for the Extreme Value Distribution Computational Statistics Quarterly, 2, 113-125. Physica-Verlag, Heidelberg.

ACHCAR, J. A.; LOUZADA, N. F. (1992). A Bayesian Approach for Accelerated Life Tests Considering the Weibull Distribution, Computational Statistics, 7: 355369.

ACHCAR, J. A. (1993). Some Aspects of Reparametrization for Statistical Models. Notas do ICMSC, Série Estatística, $n^{\circ} 001$.

ACHCAR, J. A; DAMASCENO, V. L. (1994). A Useful Reparametrization for the Survival Function Considering an Exponential Regression Model. Notas do ICMSC, Série Estatística, $\mathrm{n}^{\mathrm{0}} 007$.

ACHCAR, J. A.; DAMASCENO, V. L. (1994). Extreme Value regression Models: An Uselful Reparametrization for the Survival Function. Notas do ICMSC, Série Estatística, $\mathbf{n}^{\circ} 012$. 
AITCHISON, J. ; DUNSMORE, I. R. (1975). Statistical Prediction Analysis. Cambridge : University Press.

ANSCOMBE, F. J.(1964). Normal Likelihood Functions, Ann. Inst. Stat. Math., 6,119.

ARANDA-ORDAZ, F. J. (1981). On two families of transformations to additivity for binary response data, Biometrika, 68, 357-363.

ARMITAGE, P. ; GEHAN, E. A. (1974). Statistical Methods for in Sequential Analysis, Technometrics, 18, 301- 306.

ATKINSON, A. C. (1985). Plots, Transformations and Regressions. Oxford Clarendon Press.

BARLOW, R. E. ; PROSCHAN, F. (1979). Inference for the Exponential Life Distribution. Research Report, ORC 79-16, Operations Research Center, University of California, Berkeley.

BICKEL, P.J. ; DOKSUM, K. A. (1977). Mathematical Statistics. Basic Ideas and Selected Topics. San Francisco, Holden. Day.

BOLFARINE, H. ; RODRIGUES, J. ; ACHCAR, J. A. (1991). Análise de Sobrevivência. Rio de Janeiro, IM-UFRJ,112p. (Notas do Minicurso da II Escola de Modelos de Regressão).

BOX, G. E. P.; TIAO, G. C. (1973). Bayesian Inference in Statistical Analysis. New York, Addison. Wesley. 
BUKLEY, J. ; JAMES, I. (1979). Linear Regression With Censored Data. Biometrika, 66, 3. pp. 429-436.

CASELLA,G.; GEORGE, E.I. (1992). Explaining the Gibbs Sampler. The American Statistician, 46,3, 167-174.

COX, D. R. ; SNELL, E. J. (1968). A General Definition of Residuals. J. R. Stat. Soc. $B, 30,248-275$.

COX, D. R. (1972a). Regression Models and Life Tables (With Discusion ). Journal Royal. Statistics. Society, 27, 4-9.

FEIGL, P. ; ZELEN, M. (1965). Estimation of Exponential Survival Probabilities With Concomitant Information. Biometrics, 21, 826-837.

GELFAND, A. E. ; SMITH, A. F. M. (1990). Sampling Based Approaches to Calculating Marginal Densities, Journal of the American Statistical Association, 85, 398- 409 .

GLASSER, M. (1967). Exponential, Survival With Covariance, Journal of the American Statistical Associations, 62, 561- 568.

GLEN, L. H. (1975). Maximum Likelihood Estimation. Basead on Censored Samples, Technometrics, vol. 17, no.2, 247-254. 
GREENBERG, R. ; BAYARD, S. ; BYAR, D. (1974). Selecting Concomitant Variables Using a Likelihood Ratio Step-Down Procedure and a Method of Testing Goodness of Fit of an Exponential Survival Model, Biometrics, 30, 601-608.

GUERRERO, V. M. ; JOHNSON, R. A. (1982). Use of the Box- Cox Transformation With Binary Response Models, Biometrika, 69, 309-314.

HILLS, S. E. ; SMiTH, A. F. M. (1993). Diagnostics of Posterior Nonnormality in Bayesian Inference, Biometrika, 80, 1, pp, 61-74.

KASS, R. E. ; SLATE, E. H. (1992). Reparametrization and Diagnostics of Posterior Nonnormality. in Bayesian Statistics 4, Ed. J.M. Bernardo, J. O. Berger, A.P. Dawid and A. F. M. Smith, pp. 289-306. Oxford University Press.

KALBFLEISCH, J.D. ; PRENTICE, R. L. (1980). The Statistical Analysis of Failure Time Data. New York, John Wiley \& Sons.

KLEIN, J. P. and BASU, A. P. (1981). Webull Accelerated Life Tests When There are Competing Causes of Failure, Communications in Statistics- Theory and Methods, A10, 20, 2073-2100.

KLOEK, T. ; VAN DIJK, H. K. (1978). Bayesian Estimates of Equation System Parameters: an Aplication of Integration by Monte Carlo, Econometrica, 46, 119.

KOUL, H. SUSARLA, V. ; RYZIN, J.V. (1981). Regression Analysis With Randomly Right-Censored Data, The Annals of Statistics, vol. 9, no. 6, 1276-1288. 
LAWLESS, J. F. (1976). Confidence Interval Estimation in the Inverse Power Law Model. App. Stat. , 25, 128-138.

LAWLESS, J. F. (1980). Inference in the Generalized Gamma and Log Gamma Distributions, Technometrics, vol. 22, no.3, 409-419.

LAWLESS, J. F. (1982). Statistical Model and Methods for Lifetime Data, New York, John Wiley \& Sons.

LEONARD, T. ; HSU, J.S.J. ; TSUI, K. W. (1989). Bayesian Marginal Inference. Journal of the American Statistical Association. 84, 408, 1051-1058.

LINDLEY, D. V. (1980). Appoximate Bayesian Methods, Bayesian Statistics, Procedings of the First International Meeting Held in Valencia (eds. J. F. M. Smith ). Valencia : University Press.

LIN, L. K. ; VONESH, E. F. (1989). An Empirical Nonlinear Data-Fitting Approach for Transforming Data to Normality, The American Statistical Association, vol.43, $\mathrm{n}^{\circ} 4273-243$.

LOUZADA, N. F. (1991). Testes de Sobrevivência Acelerados: Uma Análise Bayesiana do Modelo de Eyring. Dissertação (Mestrado em Ciências de Computação e Matemática Computacional)- Instituto de Ciências Matemática de São Carlos, Universidade de São Paulo.

MARTZ, H. F. ; WALLER, R. A. (1982). Bayesian Reliability Analysis. New York, John Wiley \& Sons. 
MANN, N. R. ; SCHAFER, R. E. ; SINGPURWALLA, N. D. (1974). Methods for Statistical Analysis of Reliability and Life Data, New York: John Wiley.

MILLER, R. G. (1981). Survival Analysis. New York, John Wiley \& Sons.

MOOD, A. M. ; GRAYBILL, F. A ; BOES, D. C. (1974). Introdutions to the Theory of Statistics, 3a. Ed. New York, Mcgraw-Hill.

NAYLOR, J. C. ; SMTTH, A. F. M. (1982). Applications of a Method for the Efficient Computation of Posterior Distributions, Applied Statistics, 31, 214-225.

NELSON, W. B. (1970). Statistical Methods for Accelerated Life Tests Data- The Inverse Power Law Model, General Electric Co. Corp. Research \& Development, TIS Report 71-6-001.

NELSON, W. (1971). Analysis of Accelerated Life Tests Data- Part 1: The Arrhenius Models and Graphical Methods, IEEE Transactions on Electrical Insulation, EI-6, 4, 165-181.

NELSON, W. (1972). Graphical Analysis of Accelerated Life Tests Data with the Inverse Power Law, IEEE Transactions on Reliability R-21, 2-11.

NELSON, W. (1990). Accelerated Testing: Statistical Models, Test Plans and Data Analysis. New York: John Wiley.

PETO, R. ; LEE, P. (1973). Weibull Distributions for Continuous Carcinogenisis Experiments. Biometrics, 29, 457-470. 
PRESS, S.J. (1989). Bayesian Statistics: Models and Applications, New York, John Wiley \& Sons.

PRENTICE. R. L. (1973). Exponential Survival With Censoring and Explanatory Variable. Biometrika, 60, 279-288.

SCHMEE, J. ; HAHN, G. J. (1979). A Simple Method for Regression Analisis With Censored Data. Technometrics, vol. 21, no. 4, 417-432.

SPROTT, D.A. (1973). Normal Likelihoods and Their Relation to Large Sample Theory of Estimation. Biometrika, 60,3, 457-465.

SPROT , D. A. (1980). Maximum Likelihood in Small Samples: Estimation in the Presence of Nuisance Parameters, Biometrika, 67, 515-523.

TIERNEY, L. ; KADANE , J. B. (1986). Accurate Aproximations for Posterior Posterior Moments and Marginal Densities, Journal of The American Statistical Association, 81, 82-86.

TIERNEY, L. ; KASS, R. E. ; KADANE, J. B. (1989). Aproximate Marginal Densities for Nonlinear Funtions, Biometrika, 76, 425-433.

ZELNER, A. ; ROSSI, P. E. (1982). Bayesian Analysis of Dichotomous Quantal Response Models, Technical Report, University of Chicago, Graduate School Business. 
ZIPPIN, C. ; ARMITAGE, P. (1966). Use of Concomitant Variables and. Incomplet Survival Informations in the Estimation of an Exponential Survival Parameter, Biometrics, 22, 665-672. 